\title{
Cleavable $\beta$-Cyanoethyl Isocyanide in the Ugi Tetrazole Reaction
}

Edwin $\mathrm{Kroon}^{\dagger}$, Katarzyna Kurpiewska $^{\ddagger}$, Justyna Kalinowska-Tłuścik ${ }^{\ddagger}$, Alexander Dömling $^{\dagger *}$

${ }^{+}$University of Groningen, Department of Drug Design, A. Deusinglaan 1, 9713 AV

Groningen, The Netherlands.

* Jagiellonian University, Department of Crystal Chemistry and Crystal Physics, Ingardena 3, 30-060 Krakow, Poland.

*a.s.s.domling@rug.nl

\section{Table of Contents}

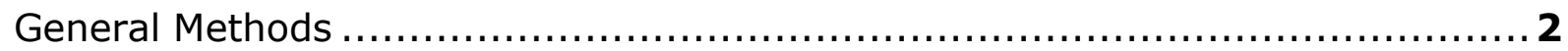

Experimental Procedure and Characterization of $\beta$-Cyanoethyl Isocyanide ........ 3

General Experimental Procedure and Characterization for Tetrazoles $5 \mathbf{a}-\mathbf{k} \ldots \ldots \mathbf{4}$

General Experimental Procedure and Characterization for $1 H$-Tetrazoles $6 \mathbf{a - k} . .8$

Experimental Procedures and Characterizations for Compounds 7, 8, and 9 ...11

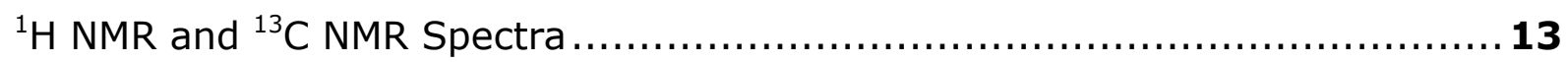

Single Crystal X-Ray Structure Determination of Compound $5 \mathbf{c} \ldots \ldots \ldots \ldots \ldots \ldots .68$

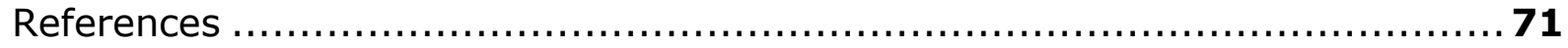




\section{General Methods}

Reagents were available from commercial suppliers and used without any purification unless otherwise noted. Thin layer chromatography was performed on Fluka precoated silica gel plates $(0.20 \mathrm{~mm}$ thick, particle size $25 \mu \mathrm{m})$. Melting points were measured on an Electrothermal IA8103 melting point apparatus. IR was recorded on a Thermo Scientific Nicolet 380 FT-IR. Nuclear magnetic resonance spectra were recorded on a Bruker Avance 500 spectrometer. Chemical shifts for ${ }^{1} \mathrm{H}$ NMR were reported relative to TMS ( $\delta 0 \mathrm{ppm}$ ) or internal solvent peak (DMSO $\delta 2.50 \mathrm{ppm}, \mathrm{CD}_{3} \mathrm{OD} \delta 3.31 \mathrm{ppm}$ or $\mathrm{D}_{2} \mathrm{O} \delta$ $4.79 \mathrm{ppm}$ ) and coupling constants were in hertz $(\mathrm{Hz})$. The following abbreviations were used for spin multiplicity: $\mathrm{s}=$ singlet, $\mathrm{d}=$ doublet, $\mathrm{t}=$ triplet, $\mathrm{dt}=$ double triplet, $\mathrm{ddd}=$ doublet of double doublet, $\mathrm{m}=$ multiplet, and $\mathrm{br}=$ broad. Chemical shifts for ${ }^{13} \mathrm{C} N \mathrm{NR}$ reported in ppm relative to the solvent peak $\left(\mathrm{CDCl}_{3} \delta 77.23 \mathrm{ppm}\right.$, DMSO $\delta 39.52 \mathrm{ppm}$, $\left.\mathrm{CD}_{3} \mathrm{OD} \delta 49.00 \mathrm{ppm}\right)$. Mass spectra were measured on a Waters Investigator Supercritical Fluid Chromatograph with a 3100 MS Detector (ESI) using a solvent system of methanol and $\mathrm{CO}_{2}$ on a Viridis silica gel column $(4.6 \times 250 \mathrm{~mm}, 5 \mu \mathrm{m}$ particle size $)$ and reported as $(\mathrm{m} / \mathrm{z})$. Filtrations were performed on a silica bed (Screening Devices BV, 60-200 $\mu \mathrm{m}, 60 \AA)$. Flash chromatography was performed on a Grace Reveleris X2 using Grace Reveleris Silica columns (12g) and a gradient of petroleum ether/ethyl acetate (0$100 \%$ ) or dichloromethane/methanol (0-40\%) was applied. 


\section{Experimental Procedure and Characterization of $\beta$-Cyanoethyl Isocyanide}

\section{N-(2-cyanoethyl)formamide (4)}

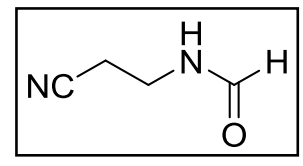

A solution of 3-aminopropionitrile ( $203 \mathrm{mmol}, 15 \mathrm{~mL}$ ) in ethyl formate (200 $\mathrm{mL}$ ) was refluxed for five hours and subsequently stirred at room temperature overnight. The reaction was concentrated in vacuo and further dried under high vacuum. The product was obtained as a light yellow oil $(19.5 \mathrm{~g}$, $199 \mathrm{mmol}, 98 \%){ }^{1} \mathrm{H}$ NMR $\left(500 \mathrm{MHz} \mathrm{CDCl}_{3}\right) \delta 8.21(\mathrm{~s}, 1 \mathrm{H}), 7.35(\mathrm{br}, 1 \mathrm{H}),(\mathrm{q}, \mathrm{J}=12.7$, $6.3,2 \mathrm{H}), 2.66(\mathrm{t}, \mathrm{J}=6.4,2 \mathrm{H}) .{ }^{13} \mathrm{C} N M R\left(126 \mathrm{MHz}, \mathrm{CDCl}_{3}\right) \delta 165.00,162.25,118.29$, $117.67,37.80,34.13,20.36,18.23$ (a mixture of amide rotamers is observed).

\section{3-isocyanopropanenitrile (2)}

NC NC $\begin{aligned} & \text { To a solution of } \mathrm{N} \text {-(2-cyanoethyl)formamide }(19.5 \mathrm{~g}, 199 \mathrm{mmol}) \text { in } \mathrm{CH}_{2} \mathrm{Cl}_{2} \\ & (400 \mathrm{~mL}) \text { was added } \mathrm{Et}_{3} \mathrm{~N}(1.0 \mathrm{~mol}, 5.0 \text { equiv., } 139 \mathrm{~mL}) \text {. The mixture was }\end{aligned}$ cooled to $-5{ }^{\circ} \mathrm{C}$ at which $\mathrm{POCl}_{3}(209 \mathrm{mmol}, 1.05$ equiv., $19.5 \mathrm{~mL}$ ) was added drop wise over 60 minutes maintaining the temperature below $0{ }^{\circ} \mathrm{C}$. After the addition the reaction was stirred at $0^{\circ} \mathrm{C}$ for an additional hour. An aqueous solution of $\mathrm{Na}_{2} \mathrm{CO}_{3}(0.6 \mathrm{M}, 100 \mathrm{~mL})$ was added carefully while the temperature increased to $20^{\circ} \mathrm{C}$. Additional water was added until all salts were dissolved $(\sim 300 \mathrm{~mL})$. The mixture was transferred to a separatory funnel and the organic layer was separated. The water layer was extracted with dichloromethane $(200 \mathrm{~mL})$. The combined organic layers were washed with brine $(100 \mathrm{~mL})$, dried over $\mathrm{MgSO}_{4}$, and concentrated in vacuo. The crude product was purified by filtration over silica $\left(100 \% \mathrm{CH}_{2} \mathrm{Cl}_{2}\right)$ and after evaporation of the solvent obtained as a pale yellow oil ( $11 \mathrm{~g}, 138 \mathrm{mmol}, 69 \%$ ) which solidified upon standing. A pure sample (white solid) was obtained by trituration of a $\mathrm{CH}_{2} \mathrm{Cl}_{2}$ solution with petroleum ether at 0 ${ }^{\circ} \mathrm{C}$. Melting point: $32{ }^{\circ} \mathrm{C}$ (lit. ${ }^{1} 32{ }^{\circ} \mathrm{C}$ ). IR (neat, $v, \mathrm{~cm}^{-1}$ ): $2256(\mathrm{w}), 2151(\mathrm{~s}), 1453(\mathrm{~m})$, $1425(m), 1358(m), 1335(w), 1259(w), 1219(w), 1015(m), 978(m), 926(w), 818$ $(\mathrm{m}, \mathrm{br}), 586(\mathrm{~m}) .{ }^{1} \mathrm{H}$ NMR $\left(500 \mathrm{MHz} \mathrm{CDCl}_{3}\right) \delta 3.72(\mathrm{t}, \mathrm{J}=6.6,2 \mathrm{H}), 2.81(\mathrm{t}, \mathrm{J}=6.5$, $2 \mathrm{H}) .{ }^{13} \mathrm{C}$ NMR $\left(126 \mathrm{MHz}, \mathrm{CDCl}_{3}\right) \delta 160.01,115.92,37.68,18.85$. 


\section{General Experimental Procedure and Characterization for Tetrazoles}

5a-k

\section{General procedure:}

A solution of aldehyde or ketone $(1.05 \mathrm{mmol})$ and amine $(1.0 \mathrm{mmol})$ in methanol $(1 \mathrm{~mL})$ was stirred at room temperature for 15 minutes. Subsequently, isocyanide $(1.0 \mathrm{mmol}, 80$ $\mathrm{mg})$ and TMS azide $(1.0 \mathrm{mmol}, 133 \mu \mathrm{l})$ were added and the reaction was stirred at room temperature overnight. The reaction was concentrated in vacuo and purified by column chromatography.

\section{3-(5-((butylamino)(4-isopropylphenyl)methyl)-1H-tetrazol-1-yl)propanenitrile}

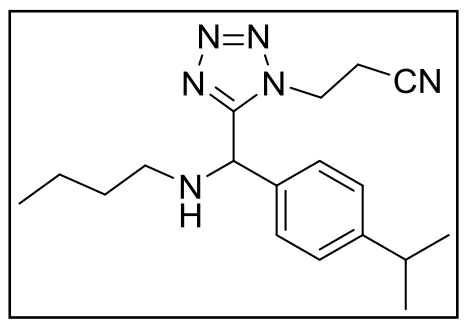

(5a). Obtained from $n$-butylamine $(99 \mu \mathrm{L})$ and 4isopropylbenzaldehyde $(159 \mu \mathrm{L})$ as a pale yellow oil $(154 \mathrm{mg}$, $0.47 \mathrm{mmol}, 47 \%) . R_{\mathrm{F}}=0.33$ (40\% EtOAc/petroleum ether). ${ }^{1} \mathrm{H}$ NMR (500 MHz, $\left.\mathrm{CDCl}_{3}\right) \delta 7.27(\mathrm{dd}, \mathrm{J}=17.5,8.1,4 \mathrm{H})$, $5.39(\mathrm{~s}, 1 \mathrm{H}), 4.74-4.57(\mathrm{~m}, 2 \mathrm{H}), 2.91$ (quint, $\mathrm{J}=7.0,1 \mathrm{H})$,

$2.85-2.60(\mathrm{~m}, 4 \mathrm{H}), 2.52(\mathrm{dt}, \mathrm{J}=11.4,7.2,1 \mathrm{H}), 1.95(\mathrm{br}, 1 \mathrm{H}), 1.61-1.44(\mathrm{~m}, 2 \mathrm{H})$, $1.44-1.28(\mathrm{~m}, 2 \mathrm{H}), 1.23(\mathrm{~d}, \mathrm{~J}=7.0,6 \mathrm{H}), 0.89(\mathrm{t}, \mathrm{J}=7.4,3 \mathrm{H}) .{ }^{13} \mathrm{C} \mathrm{NMR}(126 \mathrm{MHz}$, $\left.\mathrm{CDCl}_{3}\right) \delta 156.14,149.69,134.63,127.41,126.91,116.04,57.46,47.83,43.03,33.79$, $32.01,23.90,20.37,17.92,13.96$. MS (ESI): $m / z(\%): 327.28(100)\left[M^{+}+H\right]$.

3-(5-(cyclohexyl(mesitylamino)methyl)-1H-tetrazol-1-yl)propanenitrile (5b).

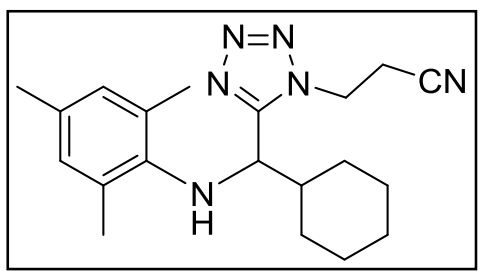
Obtained from cyclohexylcarboxaldehyde $(127 \mu \mathrm{L})$ and 2,4,6-trimethylaniline $(140 \mu \mathrm{L})$ as a white solid (122 $\mathrm{mg}$, $0.35 \mathrm{mmol}, 35 \%) \cdot R_{\mathrm{F}}=0.37$ (25\% EtOAc/petroleum ether). ${ }^{1} \mathrm{H}$ NMR $\left(500 \mathrm{MHz}, \mathrm{CDCl}_{3}\right) \delta 6.77(\mathrm{~s}, 2 \mathrm{H}), 4.04-3.85(\mathrm{~m}$, $2 \mathrm{H}), 3.70(\mathrm{ddd}, \mathrm{J}=13.7,8.9,4.5,1 \mathrm{H}), 3.27(\mathrm{~d}, \mathrm{~J}=12.3$,

$1 \mathrm{H}), 2.76-2.57(\mathrm{~m}, 2 \mathrm{H}), 2.44-2.29(\mathrm{~m}, 1 \mathrm{H}), 2.20(\mathrm{~s}, 3 \mathrm{H}), 2.01(\mathrm{~s}, 6 \mathrm{H}), 1.94-1.81$ $(\mathrm{m}, 2 \mathrm{H}), 1.80-1.67(\mathrm{~m}, 2 \mathrm{H}), 1.52-1.13(\mathrm{~m}, 5 \mathrm{H}), 1.10-0.90(\mathrm{~m}, 1 \mathrm{H}) .{ }^{13} \mathrm{C}$ NMR $(126$ $\left.\mathrm{MHz}_{1} \mathrm{CDCl}_{3}\right) \delta 157.43,140.35,133.54,130.82,130.29,115.64,56.86,42.10,42.02$, $30.59,30.41,26.56,26.19,26.08,20.68,18.19,18.07$. MS (ESI): $m / z(\%): 375.33$ $(100)\left[M^{+}+\mathrm{Na}\right]$. 


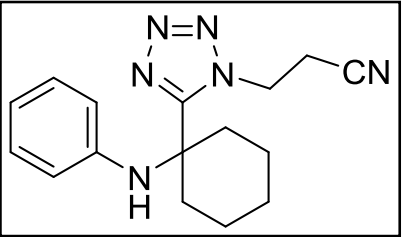

Obtained from cyclohexanone $(109 \mu \mathrm{L})$ and aniline $(91 \mu \mathrm{L})$ as a white solid (189 mg, $0.64 \mathrm{mmol}, 64 \%) . R_{\mathrm{F}}=0.50(60 \%$ EtOAc/petroleum ether). ${ }^{1} \mathrm{H}$ NMR $\left(500 \mathrm{MHz}_{1} \mathrm{CDCl}_{3}\right) \delta 7.09(\mathrm{t}, \mathrm{J}=$ $7.5,2 \mathrm{H}), 6.79(\mathrm{t}, \mathrm{J}=7.4,1 \mathrm{H}), 6.25(\mathrm{~d}, \mathrm{~J}=7.7,2 \mathrm{H}), 4.86(\mathrm{t}, \mathrm{J}$ $=7.4,2 \mathrm{H}), 4.18(\mathrm{~s}, 1 \mathrm{H}), 2.81(\mathrm{t}, \mathrm{J}=7.4,2 \mathrm{H}), 2.29(\mathrm{~d}, \mathrm{~J}=13.6,2 \mathrm{H}), 2.24-2.10(\mathrm{~m}$, $2 \mathrm{H}), 1.78-1.71(\mathrm{~m}, 3 \mathrm{H}), 1.64-1.49(\mathrm{~m}, 2 \mathrm{H}), 1.49-1.37(\mathrm{~m}, 1 \mathrm{H}) .{ }^{13} \mathrm{C} N M R(126 \mathrm{MHz}$, $\left.\mathrm{CDCl}_{3}\right) \delta 159.61,143.56,129.74,119.99,115.95,115.08,99.98,54.26,43.90,34.31$, 24.74, 20.96, 18.11. MS (ESI): $m / z(\%): 242.03(100)\left[M^{-}-\left(\mathrm{CH}_{2}\right)_{2} \mathrm{CN}\right]$.

\section{4-((1-(1-(2-cyanoethyl)-1H-tetrazol-5-yl)-2-methylpropyl)amino)benzonitrile}

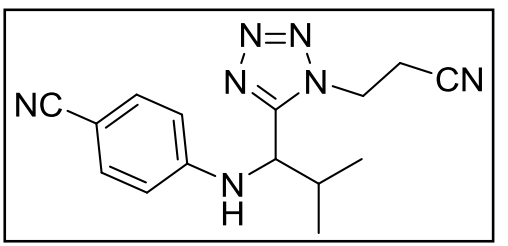

(5d). Obtained from 4-aminobenzonitrile (118 mg) and isobutyraldehyde $(96 \mu \mathrm{L})$ as a white gummy solid $(179 \mathrm{mg}$, $0.66 \mathrm{mmol}, 66 \%) . \quad R_{\mathrm{F}}=0.26(50 \% \mathrm{EtOAc} /$ petroleum ether). ${ }^{1} \mathrm{H}$ NMR $\left(500 \mathrm{MHz}, \mathrm{CDCl}_{3}\right) \delta 7.39(\mathrm{~d}, \mathrm{~J}=8.6,1 \mathrm{H})$, $6.73(\mathrm{~d}, \mathrm{~J}=8.7,1 \mathrm{H}), 5.70(\mathrm{~d}, \mathrm{~J}=7.3, \mathrm{OH}), 4.86-4.70(\mathrm{~m}, 2 \mathrm{H}), 3.06(\mathrm{qt}, \mathrm{J}=17.1,6.8$, $1 \mathrm{H}), 2.54-2.34(\mathrm{~m}, 1 \mathrm{H}), 1.16(\mathrm{~d}, \mathrm{~J}=6.6,2 \mathrm{H}), 0.96(\mathrm{~d}, \mathrm{~J}=6.7,2 \mathrm{H}) .{ }^{13} \mathrm{C} \mathrm{NMR}(126 \mathrm{MHz}$, $\left.\mathrm{CDCl}_{3}\right) \delta 155.53,150.04,134.01,120.15,116.38,112.89,100.00,54.55,43.37,32.56$, 19.34, 19.16, 18.34. MS (ESI): $m / z(\%): 241.06(100)\left[M^{-}-\left(\mathrm{CH}_{2}\right)_{2} \mathrm{CN}\right]$.

\section{3-(5-(benzo[d][1,3]dioxol-5-yl(pyrrolidin-1-yl)methyl)-1H-tetrazol-1-}

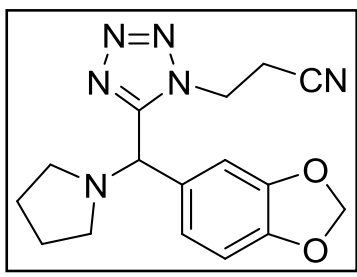

yl)propanenitrile (5e). Obtained from piperonal $(158 \mathrm{mg})$ and pyrrolidine $(84 \mu \mathrm{L})$ as a white solid $(156 \mathrm{mg}, 0.48 \mathrm{mmol}, 48 \%) . R_{\mathrm{F}}$ $=0.26$ (50\% EtOAc/petroleum ether). ${ }^{1} \mathrm{H} \mathrm{NMR}\left(500 \mathrm{MHz}, \mathrm{CDCl}_{3}\right) \delta$ $7.00-6.88(\mathrm{~m}, 2 \mathrm{H}), 6.82-6.78(\mathrm{~m}, 1 \mathrm{H}), 5.97(\mathrm{~s}, 2 \mathrm{H}), 4.99(\mathrm{~s}$, $1 \mathrm{H}), 4.85-4.66(\mathrm{~m}, 1 \mathrm{H}), 3.05-2.86(\mathrm{~m}, 1 \mathrm{H}), 2.84-2.72(\mathrm{~m}$, $2 \mathrm{H}), 2.72-2.57(\mathrm{~m}, 2 \mathrm{H}), 2.49-2.33(\mathrm{~m}, 2 \mathrm{H}), 1.93-1.76(\mathrm{~m}, 4 \mathrm{H}) .{ }^{13} \mathrm{C} \mathrm{NMR}(126 \mathrm{MHz}$, $\left.\mathrm{CDCl}_{3}\right) \delta 155.75,148.61,148.10,130.12,121.44,115.94,108.77,108.31,101.71$, $64.33,53.10,43.18,23.65,18.01$. MS (ESI): $m / z(\%): 327.33(100)\left[M^{+}+H\right], 349.24$ (55) $\left[M^{+}+\mathrm{Na}\right]$. 


\section{3-(5-(morpholino(naphthalen-1-yI)methyl)-1H-tetrazol-1-yl)propanenitrile}

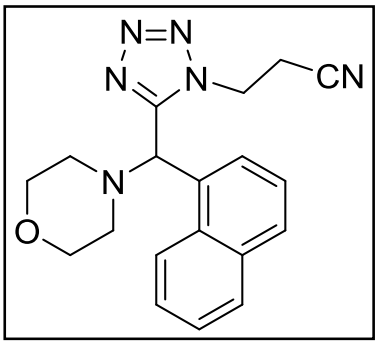

(5f). Obtained from 1-naphthaldehyde (143 $\mu \mathrm{L})$ and morpholine $(87 \mu \mathrm{L})$ as a pale yellow oil $(125 \mathrm{mg}, 0.36 \mathrm{mmol}, 36 \%) . R_{\mathrm{F}}=$ 0.26 (50\% EtOAc/petroleum ether). ${ }^{1} \mathrm{H}$ NMR $\left(500 \mathrm{MHz}, \mathrm{CDCl}_{3}\right) \delta$ $8.43(\mathrm{~d}, \mathrm{~J}=8.5,1 \mathrm{H}), 7.87(\mathrm{dd}, \mathrm{J}=8.1,4.2,2 \mathrm{H}), 7.68(\mathrm{~d}, \mathrm{~J}=$ $7.2,1 \mathrm{H}), 7.57(\mathrm{t}, \mathrm{J}=7.7,1 \mathrm{H}), 7.52(\mathrm{t}, \mathrm{J}=7.6,2 \mathrm{H}), 5.87(\mathrm{~s}, 1 \mathrm{H})$, $4.51(\mathrm{ddq}, \mathrm{J}=21.0,14.0,7.1,2 \mathrm{H}), 3.82-3.71(\mathrm{~m}, 4 \mathrm{H}), 2.91-$ $2.76(\mathrm{~m}, 2 \mathrm{H}), 2.69(\mathrm{dt}, \mathrm{J}=16.9,7.0,1 \mathrm{H}), 2.53(\mathrm{dt}, \mathrm{J}=16.9,7.1,1 \mathrm{H}), 2.49-2.38(\mathrm{~m}$, $2 \mathrm{H}) .{ }^{13} \mathrm{C}$ NMR $\left(126 \mathrm{MHz} \mathrm{CDCl}_{3}\right) \delta 154.03,134.36,131.35,130.22,129.79,129.19$, $127.46,126.68,126.04,125.08,123.34,115.87,67.01,61.79,52.12,43.25,17.85$. MS (ESI): $m / z(\%): 371.25(100)\left[M^{+}+\mathrm{Na}\right]$.

tert-butyl 4-(1-(1-(2-cyanoethyl)-1H-tetrazol-5-yl)-2-phenylethyl)piperazine-1-

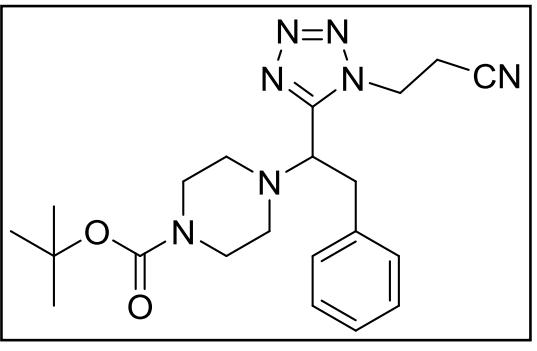
carboxylate $\mathbf{( 5 g )}$. Obtained from phenylacetaldehyde $(123 \mu \mathrm{L})$ and 1-Boc-piperazine $(186 \mathrm{mg})$ as an off-white solid (226 mg, $0.55 \mathrm{mmol}, 55 \%) . R_{\mathrm{F}}=0.27(50 \%$ EtOAc/petroleum ether). ${ }^{1} \mathrm{H}$ NMR $\left(500 \mathrm{MHz} \mathrm{CDCl}_{3}\right) \delta$ $7.32-7.16(\mathrm{~m}, 3 \mathrm{H}), 7.13(\mathrm{~d}, J=6.9,2 \mathrm{H}), 4.51-4.24$ $(\mathrm{m}, 2 \mathrm{H}), 4.15(\mathrm{dd}, \mathrm{J}=10.2,3.8,1 \mathrm{H}), 3.55-3.36(\mathrm{~m}$, $5 \mathrm{H}), 3.30(\mathrm{dd}, \mathrm{J}=13.2,3.7,1 \mathrm{H}), 2.96-2.79(\mathrm{~m}, 1 \mathrm{H}), 2.70(\mathrm{br}, 2 \mathrm{H}), 2.65-2.50(\mathrm{~m}$, $3 \mathrm{H}), 1.45(\mathrm{~s}, 9 \mathrm{H}) .{ }^{13} \mathrm{C}$ NMR $\left(126 \mathrm{MHz}, \mathrm{CDCl}_{3}\right) \delta 154.70,154.15,137.51,129.54,129.06$, $127.23,115.80,80.27,62.24,49.23,42.63,33.86,28.58,18.24$. MS (ESI): $m / z(\%)$ : $334.26(100)\left[(M-B o c)^{+}+\mathrm{Na}\right], 434.33(75)\left[M^{+}+\mathrm{Na}\right]$.

3-(5-((tritylamino)methyl)-1H-tetrazol-1-yl)propanenitrile (5h). Obtained from

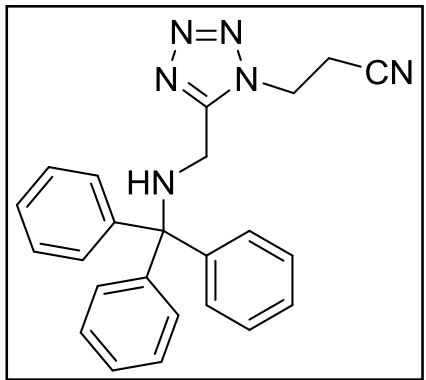
paraformaldehyde $(32 \mathrm{mg})$ and tritylamine $(259 \mathrm{mg})$ as a white solid (269 mg, $0.68 \mathrm{mmol}, 68 \%) . \quad R_{\mathrm{F}}=0.24(40 \%$ EtOAc/petroleum ether). ${ }^{1} \mathrm{H}$ NMR $\left(500 \mathrm{MHz} \mathrm{CDCl}_{3}\right) \delta 7.45$ (d, J $=7.8,6 \mathrm{H}), 7.33(\mathrm{t}, \mathrm{J}=7.6,6 \mathrm{H}), 7.29-7.17(\mathrm{~m}, 3 \mathrm{H}), 4.44(\mathrm{t}$, $\mathrm{J}=6.9,2 \mathrm{H}), 3.85(\mathrm{~d}, \mathrm{~J}=7.6,2 \mathrm{H}), 2.94(\mathrm{t}, \mathrm{J}=6.9,2 \mathrm{H}) .{ }^{13} \mathrm{C}$ NMR $\left(126 \mathrm{MHz}, \mathrm{CDCl}_{3}\right) \delta 154.11,144.24,128.55,128.32$ ， $127.14,115.58,71.33,42.61,36.87,18.62$. MS (ESI): m/z (\%): 340.33 (100)[M-$\left.\left(\mathrm{CH}_{2}\right)_{2} \mathrm{CN}\right]$. 


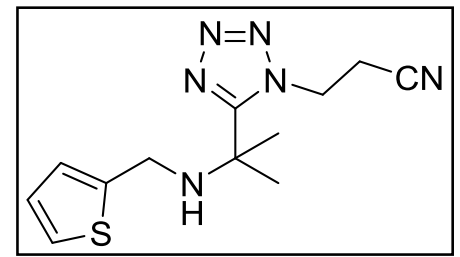

yl)propanenitrile (5i). Obtained from acetone $(88 \mu \mathrm{L})$ and 2-thiophenemethylamine $(103 \mu \mathrm{L})$ as a pale yellow solid (180 $\mathrm{mg}, 0.65 \mathrm{mmol}, 65 \%) . R_{\mathrm{F}}=0.25(40 \% \mathrm{EtOAc} /$ petroleum ether). ${ }^{1} \mathrm{H}$ NMR $\left(500 \mathrm{MHz}, \mathrm{CDCl}_{3}\right) \delta 7.23$ (dd, $\mathrm{J}=5.1,1.1$,

$1 \mathrm{H}), 6.94(\mathrm{dd}, \mathrm{J}=5.1,3.5,1 \mathrm{H}), 6.86(\mathrm{dd}, \mathrm{J}=3.3,0.8,1 \mathrm{H}), 5.11(\mathrm{t}, \mathrm{J}=7.3,2 \mathrm{H}), 3.80$ $(\mathrm{d}, \mathrm{J}=5.8,2 \mathrm{H}), 3.12(\mathrm{~d}, \mathrm{~J}=7.3,2 \mathrm{H}), 1.75(\mathrm{~s}, 6 \mathrm{H}) .1 .62(\mathrm{br}, 1 \mathrm{H}) .{ }^{13} \mathrm{C} \mathrm{NMR}(126 \mathrm{MHz}$, $\left.\mathrm{CDCl}_{3}\right) \delta 158.64,142.53,127.00,124.92,124.73,116.02,53.74,44.08,42.71,27.66$, 18.39. MS (ESI): $m / z(\%): 299.22(100)\left[M^{+}+\mathrm{Na}\right]$.

\section{3-(5-(benzo[b]thiophen-2-yl(tert-butylamino)methyl)-1H-tetrazol-1-}

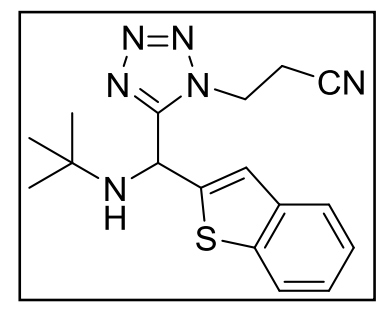

yl)propanenitrile (5j). Obtained from 2-benzothiophene (170 $\mathrm{mg}$ ) and tert-butylamine $(105 \mu \mathrm{L})$ as a white solid (82 $\mathrm{mg}, 0.24$ $\mathrm{mmol}, 24 \%) . R_{\mathrm{F}}=0.25$ (25\% EtOAc/petroleum ether). ${ }^{1} \mathrm{H}$ NMR $\left(500 \mathrm{MHz} \mathrm{CDCl}_{3}\right) \delta 7.86-7.75(\mathrm{~m}, 1 \mathrm{H}), 7.75-7.63(\mathrm{~m}, 1 \mathrm{H})$, $7.44-7.30(\mathrm{~m}, 1 \mathrm{H}), 7.01(\mathrm{~s}, 1 \mathrm{H}), 6.06(\mathrm{~s}, 1 \mathrm{H}), 5.01-4.77(\mathrm{~m}$, $2 \mathrm{H}), 3.08(\mathrm{ddd}, \mathrm{J}=16.8,8.0,6.8,1 \mathrm{H}), 2.97(\mathrm{ddd}, \mathrm{J}=16.8,8.0,6.5,1 \mathrm{H}), 1.87(\mathrm{~s}, 1 \mathrm{H})$, $1.14(\mathrm{~s}, 9 \mathrm{H}) .{ }^{13} \mathrm{C}$ NMR $\left(126 \mathrm{MHz} \mathrm{CDCl}_{3}\right) \delta 156.79,143.38,139.28,139.22,125.46$, $125.30,124.17,122.64,122.24,116.06,52.88,49.63,43.89,29.45,18.09$. MS (ESI): $m / z(\%): 363.24(100)\left[M^{+}+\mathrm{Na}\right]$.

\section{3-(5-(((2-hydroxyethyl)amino)(pyridin-3-yl)methyl)-1H-tetrazol-1-}

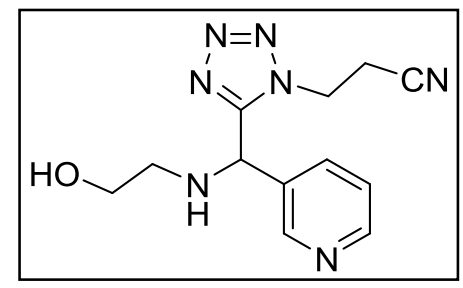

yl)propanenitrile (5k). Obtained from ethanolamine $(60 \mu \mathrm{L})$ and 3-pyridinecarboxaldehyde $(122 \mu \mathrm{L})$ as a pale yellow oil (144 mg, $0.53 \mathrm{mmol}, 53 \%) . R_{\mathrm{F}}=0.35\left(15 \% \mathrm{MeOH} / \mathrm{CH}_{2} \mathrm{Cl}_{2}\right)$. ${ }^{1} \mathrm{H}$ NMR $\left(500 \mathrm{MHz}, \mathrm{CDCl}_{3}\right) \delta 8.66(\mathrm{~d}, \mathrm{~J}=1.8,1 \mathrm{H}), 8.53(\mathrm{~d}, \mathrm{~J}=$ $4.7,1 \mathrm{H}), 7.84(\mathrm{~d}, \mathrm{~J}=8.0,1 \mathrm{H}), 7.35(\mathrm{dd}, \mathrm{J}=7.9,4.8,1 \mathrm{H})$, $5.49(\mathrm{~s}, 1 \mathrm{H}), 4.97-4.66(\mathrm{~m}, 2 \mathrm{H}), 3.81-3.50(\mathrm{~m}, 2 \mathrm{H}), 3.21-2.98(\mathrm{~m}, 2 \mathrm{H}), 2.84-$ $2.54(\mathrm{~m}, 2 \mathrm{H}) .{ }^{13} \mathrm{C}$ NMR $\left(126 \mathrm{MHz} \mathrm{CDCl}_{3}\right) \delta 155.56,149.50,148.76,135.40,133.08$, $123.82,116.54,60.85,54.56,48.96,43.03,18.06$. MS (ESI): $m / z(\%): 296.22$ $(100)\left[M^{+}+\mathrm{Na}\right], 274.24(40)\left[M^{+}+\mathrm{H}\right]$. 


\section{General Experimental Procedure and Characterization for $\mathbf{1 H}$-Tetrazoles}

6a-k

\section{General procedure:}

To a solution of protected tetrazole $(50 \mathrm{mg})$ in $\mathrm{THF} / \mathrm{H}_{2} \mathrm{O}(5: 1,1 \mathrm{~mL})$ was added $\mathrm{LiOH}(2.0$ equiv.). The resulting suspension was stirred at room temperature for $30 \mathrm{~min}$. The reaction was concentrated to dryness and water $(500 \mu \mathrm{L})$ was added. The solution was cooled to $0{ }^{\circ} \mathrm{C}$ and acidified to $\mathrm{pH} 4-5$ with $\mathrm{HCl}(0.1 \mathrm{M})$. The precipitate was collected by filtration, washed with cold water and dried under high vacuum.

N-((4-isopropylphenyl)(1H-tetrazol-5-yl)methyl)butan-1-amine (6a). Obtained

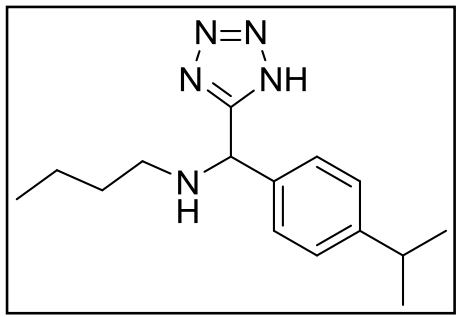

from protected tetrazole $\mathbf{5 a}$ as a white solid (35 $\mathrm{mg}, 0.13$ $\mathrm{mmol}, 84 \%) .{ }^{1} \mathrm{H}$ NMR $\left(500 \mathrm{MHz} \mathrm{CDCl}_{3}\right) \delta 7.41(\mathrm{~d}, \mathrm{~J}=8.3$, $2 \mathrm{H}), 7.11(\mathrm{~d}, \mathrm{~J}=8.2,2 \mathrm{H}), 5.91(\mathrm{~s}, 1 \mathrm{H}), 3.09-2.91(\mathrm{~m}, 1 \mathrm{H})$, $2.83(\mathrm{ddd}, \mathrm{J}=14.0,9.6,6.3,4 \mathrm{H}), 1.72-1.57(\mathrm{~m}, 1 \mathrm{H}), 1.57$ $-1.47(\mathrm{~m}, 1 \mathrm{H}), 1.23-1.17(\mathrm{~m}, 2 \mathrm{H}), 1.16(\mathrm{~d}, \mathrm{~J}=6.9,7 \mathrm{H})$, $0.74(\mathrm{t}, \mathrm{J}=7.4,3 \mathrm{H}) \cdot{ }^{13} \mathrm{C} \mathrm{NMR}\left(126 \mathrm{MHz}, \mathrm{CDCl}_{3}\right) \delta 158.00$, $150.41,131.49,128.33,127.31,58.34,46.72,33.93,28.01,23.91,23.85,19.98$, 13.55. MS (ESI): $m / z(\%): 272.25(100)\left[M^{-}-H\right]$.

N-(cyclohexyl(1H-tetrazol-5-yl)methyl)-2,4,6-trimethylaniline (6b). Obtained

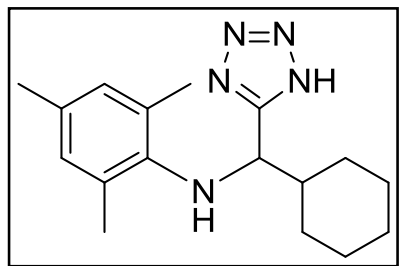
from protected tetrazole $\mathbf{5 b}$ as a white solid $(39 \mathrm{mg}, 0.13 \mathrm{mmol}$, 91\%). ${ }^{1} \mathrm{H}$ NMR (500 MHz, $\left.\mathrm{CDCl}_{3}\right) \delta 6.74(\mathrm{~s}, 2 \mathrm{H}), 4.40$ (dd, J = $9.3,6.1,1 \mathrm{H}), 2.18(\mathrm{~s}, 3 \mathrm{H}), 2.16-2.03(\mathrm{~m}, 8 \mathrm{H}), 1.85-1.76(\mathrm{~m}$, $1 \mathrm{H}), 1.70(\mathrm{t}, \mathrm{J}=14.8,2 \mathrm{H}), 1.61(\mathrm{~d}, \mathrm{~J}=11.7,1 \mathrm{H}), 1.39-1.21$ $(\mathrm{m}, 3 \mathrm{H}), 1.20-1.07(\mathrm{~s}, 1 \mathrm{H}), 1.07-0.94(\mathrm{~m}, 1 \mathrm{H}) .{ }^{13} \mathrm{C}$ NMR $(126$ $\mathrm{MHz}, \mathrm{CDCl} 3) \delta 159.04,141.40,132.07,130.21,129.14,58.39,43.01,29.73,29.28$, $26.42,26.29,26.24,20.68,18.85 . . M S(E S I): m / z(\%): 298.20(100)\left[M^{-}-H\right]$.

N-(1-(1H-tetrazol-5-yl)cyclohexyl)aniline (6c). Obtained from protected tetrazole

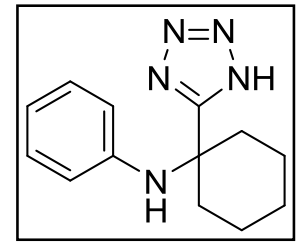
5c as an off-white solid (30 mg, $0.12 \mathrm{mmol}, 73 \%) .{ }^{1} \mathrm{H}$ NMR (500 MHz, $\left.\mathrm{CDCl}_{3}\right) \delta 7.04(\mathrm{t}, \mathrm{J}=7.9,2 \mathrm{H}), 6.72(\mathrm{t}, \mathrm{J}=7.4,1 \mathrm{H}), 6.25(\mathrm{~d}, \mathrm{~J}=8.0$, $2 \mathrm{H}), 2.21(\mathrm{~d}, \mathrm{~J}=13.4,2 \mathrm{H}), 2.14-2.02(\mathrm{~m}, 2 \mathrm{H}), 1.73-1.60(\mathrm{~m}, 3 \mathrm{H})$, $1.60-1.46(\mathrm{~m}, 2 \mathrm{H}), 1.46-1.32(\mathrm{~m}, 1 \mathrm{H}) .{ }^{13} \mathrm{C} \mathrm{NMR}\left(126 \mathrm{MHz}, \mathrm{CDCl}_{3}\right) \delta$ $163.17,143.75,129.49,119.56,115.93,54.07,34.84,25.05,21.19 . M S$ (ESI): $\mathrm{m} / \mathrm{z}$ (\%): 242.13(100)[M--H]. 
4-((2-methyl-1-(1H-tetrazol-5-yl)propyl)amino)benzonitrile (6d). Obtained from

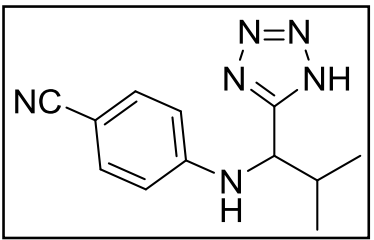
protected tetrazole $\mathbf{5 d}$ as an off-white solid $(31 \mathrm{mg}, 0.13 \mathrm{mmol}$, $76 \%) .{ }^{1} \mathrm{H}$ NMR $\left(500 \mathrm{MHz}, \mathrm{CDCl}_{3}\right) \delta 7.24(\mathrm{~d}, \mathrm{~J}=8.8,2 \mathrm{H}), 6.50(\mathrm{~d}$, $\mathrm{J}=8.8,2 \mathrm{H}), 5.35(\mathrm{~d}, \mathrm{~J}=7.2,1 \mathrm{H}), 4.86(\mathrm{t}, \mathrm{J}=6.4,1 \mathrm{H}), 2.36$ $(d q, J=13.4,6.7,1 H), 1.11(d, J=6.8,1 H), 0.96(d, J=6.8$, $1 \mathrm{H}) .{ }^{13} \mathrm{C} \mathrm{NMR}\left(126 \mathrm{MHz}, \mathrm{CDCl}_{3}\right) \delta 158.82,150.01,133.95,120.16,113.05,99.85$, 54.74, 33.41, 19.06, 18.91.MS (ESI): $m / z(\%): 241.17(100)\left[M^{-}-H\right]$.

5-(benzo[d][1,3]dioxol-5-yl(pyrrolidin-1-yl)methyl)-1H-tetrazole (6e). Obtained

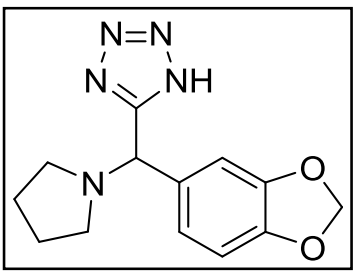
from protected tetrazole $5 \mathbf{e}$ as a white solid $(29 \mathrm{mg}, 0.11 \mathrm{mmol}$, 69\%). 1H NMR (500 MHz, CD 30 D) $\delta 7.18(s, 1 H), 7.13(d, j=8.0$, $1 \mathrm{H}), 6.87(\mathrm{~d}, \mathrm{~J}=8.0,1 \mathrm{H}), 5.99(\mathrm{~d}, \mathrm{~J}=6.7,2 \mathrm{H}), 5.68(\mathrm{~s}, 1 \mathrm{H}), 3.35$ $(\mathrm{s}, 2 \mathrm{H}), 3.26-3.09(\mathrm{~m}, 2 \mathrm{H}), 2.13-1.97(\mathrm{~m}, 4 \mathrm{H}) .{ }^{13} \mathrm{C}$ NMR $(126$ $\left.\mathrm{MHz}, \mathrm{CD}_{3} \mathrm{OD}\right) \delta 158.03,149.29,148.61,126.59,123.56,108.51$, $108.30,101.88,64.41,53.61,22.81$. MS (ESI): $m / z(\%): 272.08(100)\left[M^{-}-H^{-}\right]$.

4-(naphthalen-1-yl(1H-tetrazol-5-yl)methyl)morpholine (6f). Obtained from<smiles>c1ccc2c(C(c3nn[nH]n3)N3CCOCC3)cccc2c1</smiles>
protected tetrazole $\mathbf{5 f}$ as a white solid $(33 \mathrm{mg}, 0.11 \mathrm{mmol}, 77 \%) .{ }^{1} \mathrm{H}$ NMR $\left(500 \mathrm{MHz} \mathrm{CDCl}_{3}\right) \delta 8.44(\mathrm{~d}, \mathrm{~J}=7.4,1 \mathrm{H}), 7.90-7.80(\mathrm{~m}, 2 \mathrm{H})$, $7.78(\mathrm{~d}, \mathrm{~J}=8.2,1 \mathrm{H}), 7.51-7.42(\mathrm{~m}, 2 \mathrm{H}), 7.35(\mathrm{t}, \mathrm{J}=7.7,1 \mathrm{H}), 5.93$ $(\mathrm{s}, 1 \mathrm{H}), 3.84-3.54(\mathrm{~m}, 4 \mathrm{H}), 2.84-2.64(\mathrm{~m}, 2 \mathrm{H}), 2.60-2.38(\mathrm{~m}$, $2 \mathrm{H}) .{ }^{13} \mathrm{C}$ NMR $\left(126 \mathrm{MHz}, \mathrm{CDCl}_{3}\right) \delta 157.52,134.25,131.49,131.37$, $129.89,129.30,127.27,127.19,126.41,125.61,123.22,66.58$,

52.26. MS (ESI): $m / z(\%): 294.12(100)\left[M^{-}-H^{-}\right]$.

tert-butyl 4-(2-phenyl-1-(1H-tetrazol-5-yl)ethyl)piperazine-1-carboxylate (6g).

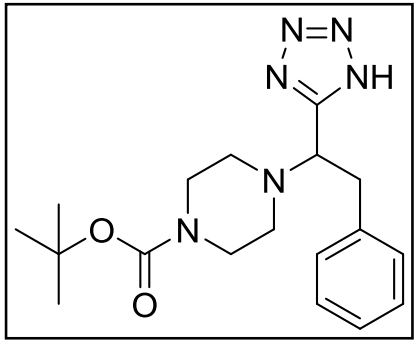
Obtained from protected tetrazole $\mathbf{5 g}$ as a white solid $(41 \mathrm{mg}$, $0.11 \mathrm{mmol}, 93 \%) .{ }^{1} \mathrm{H}$ NMR $\left(500 \mathrm{MHz}, \mathrm{CDCl}_{3}\right) \delta 7.25-7.00(\mathrm{~m}$, $5 \mathrm{H}), 4.45-4.23(\mathrm{~m}, 1 \mathrm{H}), 3.48-3.17(\mathrm{~m}, 6 \mathrm{H}), 2.51(\mathrm{dd}, \mathrm{J}=$ $36.6,4.6,4 \mathrm{H}), 1.42(\mathrm{~s}, 9 \mathrm{H}) \cdot{ }^{13} \mathrm{C} \mathrm{NMR}\left(126 \mathrm{MHz}, \mathrm{CDCl}_{3}\right) \delta$ $154.98,137.47,129.19,128.60,126.80,80.45,61.48,49.33$, 36.72, 28.46. MS (ESI): $m / z(\%): 357.18(100)\left[M^{-}-H^{-}\right]$. 
N-((1H-tetrazol-5-yl)methyl)-1,1,1-triphenylmethanamine (6h). Obtained from

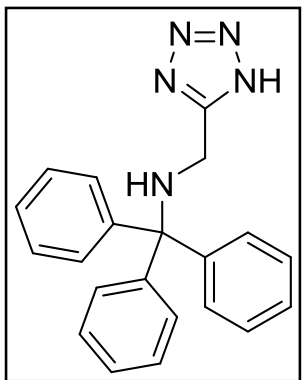
protected tetrazole $5 \mathbf{h}$ as a white solid (38 $\mathrm{mg}, 0.11 \mathrm{mmol}, 87 \%$ ). ${ }^{1} \mathrm{H}$ NMR $\left(500 \mathrm{MHz} \mathrm{CDCl}_{3}\right) \delta 7.51(\mathrm{~d}, \mathrm{~J}=7.8,6 \mathrm{H}), 7.27(\mathrm{t}, \mathrm{J}=7.6,6 \mathrm{H})$, $7.19(\mathrm{t}, \mathrm{J}=7.2,3 \mathrm{H}), 3.65(\mathrm{~s}, 2 \mathrm{H}) \cdot{ }^{13} \mathrm{C} \mathrm{NMR}(126 \mathrm{MHz}, \mathrm{DMSO}) \delta$ $155.82,145.29,128.41,127.88,126.37,70.57,38.04$. MS (ESI): $m / z$ (\%): $340.33(100)\left[M^{-}-\mathrm{H}\right]$.

2-(1H-tetrazol-5-yl)-N-(thiophen-2-ylmethyl)propan-2-amine (6i). Obtained from

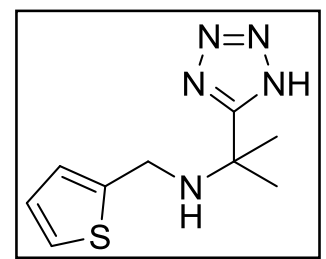

protected tetrazole $5 \mathbf{i}$ as a white solid (34 mg, $0.15 \mathrm{mmol}, 83 \%$ ). ${ }^{1} \mathrm{H}$ NMR $\left(500 \mathrm{MHz}, \mathrm{CD}_{3} \mathrm{OD}\right) \delta 7.55(\mathrm{~d}, \mathrm{~J}=5.0,1 \mathrm{H}), 7.25(\mathrm{~d}, \mathrm{~J}=3.2,1 \mathrm{H})$, $7.19-7.03(\mathrm{~m}, 1 \mathrm{H}), 4.32(\mathrm{~s}, 2 \mathrm{H}), 1.88(\mathrm{~s}, 6 \mathrm{H}) .{ }^{13} \mathrm{C} N M R(126 \mathrm{MHz}$, $\left.\mathrm{CD}_{3} \mathrm{OD}\right) \delta 163.42,134.25,131.59,129.32,128.79,59.08,42.07$, 25.66. MS (ESI): $m / z(\%): 222.09(100)\left[M^{-}-\mathrm{H}\right]$.

\section{N-(benzo[b]thiophen-2-yl(1H-tetrazol-5-yl)methyl)-2-methylpropan-2-amine}

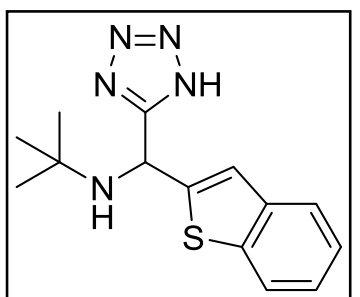

(6j). Obtained from protected tetrazole $\mathbf{5} \mathbf{j}$ as a white solid (25 $\mathrm{mg}$, $0.09 \mathrm{mmol}, 59 \%) .{ }^{1} \mathrm{H}$ NMR (500 MHz, DMSO) $\delta 8.03-7.86(\mathrm{~m}$, $1 \mathrm{H}), 7.80(\mathrm{dd}, \mathrm{J}=6.8,1.9,1 \mathrm{H}), 7.47(\mathrm{~s}, 1 \mathrm{H}), 7.40-7.24(\mathrm{~m}, 2 \mathrm{H})$, $6.08(\mathrm{~s}, 1 \mathrm{H}), 1.12(\mathrm{~s}, 9 \mathrm{H}) .{ }^{13} \mathrm{C}$ NMR (126 MHz, DMSO) $\delta$ 159.08, $139.55,138.93,124.59,124.46,123.80,123.35,122.45,49.10$,

27.44. MS (ESI): $m / z(\%): 286.09(100)\left[M^{-}-H^{-}\right]$.

2-((pyridin-3-yl(1H-tetrazol-5-yl)methyl)amino)ethan-1-ol (6k). Obtained from

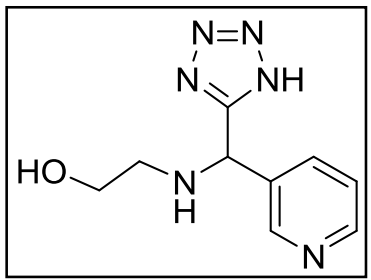

protected tetrazole $\mathbf{5 k}$ as a white solid (33 $\mathrm{mg}, 0.15 \mathrm{mmol}, 81 \%$ ). ${ }^{1} \mathrm{H}$ NMR (500 MHz, DMSO) $\delta 8.76(\mathrm{~s}, 1 \mathrm{H}), 8.56-8.54(\mathrm{~m}, 1 \mathrm{H})$, $7.98(\mathrm{~d}, \mathrm{~J}=8.0,1 \mathrm{H}), 7.43(\mathrm{dd}, \mathrm{J}=7.9,4.8,1 \mathrm{H}), 5.74(\mathrm{~s}, 1 \mathrm{H})$, $3.69-3.50(\mathrm{~m}, 3 \mathrm{H}), 2.80(\mathrm{dd}, \mathrm{J}=9.7,5.7,3 \mathrm{H}) .{ }^{13} \mathrm{C}$ NMR $(126$ $\mathrm{MHz}, \mathrm{DMSO}) \delta 157.62,149.97,149.65,136.45,132.46,123.56$,

57.39, 55.39, 48.16. MS (ESI): $m / z(\%): 219.03(100)\left[M^{-}-\mathrm{H}\right]$. 


\section{Experimental Procedures and Characterizations for Compounds 7, 8, and}

9

N-benzyl-N-(1-((2-cyanoethyl)amino)-2-methylpropyl)benzamide (7). A solution (c) of isobutyraldehyde $(91 \mu \mathrm{L}, 1.0 \mathrm{mmol})$ and benzylamine (109 $\mu \mathrm{L}, 1.0 \mathrm{mmol})$ in methanol $(1.0 \mathrm{~mL})$ was stirred at $\mathrm{rt}$ for 15 min. Then benzoic acid (122 $\mathrm{mg}, 1.0 \mathrm{mmol}$ ) and $\beta$-cyanoethyl isocyanide $(80 \mathrm{mg}, 1.0 \mathrm{mmol})$ were added and the reaction mixture stirred overnight at $\mathrm{rt}$. The solvent was evaporated and the residue purified by column chromatography. ${ }^{1} \mathrm{H}$ NMR $\left(500 \mathrm{MHz} \mathrm{CDCl}_{3}\right) \delta 8.18(\mathrm{~s}, 1 \mathrm{H}), 7.62-7.34(\mathrm{~m}, 5 \mathrm{H})$, $7.22(t, J=6.0,3 H), 7.05(d, J=5.7,2 H), 4.57(d d, J=84.4,15.6,2 H), 4.04(d, J=$ $10.9,1 \mathrm{H}), 3.45-3.14(\mathrm{~m}, 2 \mathrm{H}), 2.88-2.66(\mathrm{~m}, 1 \mathrm{H}), 2.50-2.21(\mathrm{~m}, 2 \mathrm{H}), 1.07(\mathrm{~d}, \mathrm{~J}=$ $6.4,3 \mathrm{H}), 0.99(\mathrm{~d}, \mathrm{~J}=6.3,3 \mathrm{H}) .{ }^{13} \mathrm{C}$ NMR $(126 \mathrm{MHz}, \mathrm{CDCl} 3) \delta 174.12,171.01,136.61$, $136.33,130.07,128.73,128.42,127.75,127.69,126.93,118.21,68.16,53.25,35.40$, 26.96, 19.96, 19.42, 17.82. MS (ESI): $m / z(\%): 362.13(100)\left[M^{-}-\mathrm{H}\right]$.

1-(2-phenyl-1-(1H-tetrazol-5-yl)ethyl)piperazine hydrochloride (8). To a

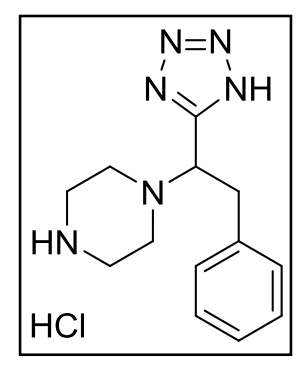
suspension of $1 \mathrm{H}$-tetrazole $\mathbf{6 g}$ ( $35 \mathrm{mg}, 0.1 \mathrm{mmol}$ ) in 1,4-dioxane ( 1 $\mathrm{mL}$ ) was added $4.0 \mathrm{M} \mathrm{HCl}$ in dioxane $(125 \mu \mathrm{L}, 0.5 \mathrm{mmol})$. The reaction became clear and was stirred at room temperature overnight. The reaction was concentrated in vacuo and ether $(5 \mathrm{~mL})$ was added to the residue. The solid was collected by filtration, washed with ether $(2 \mathrm{~mL})$ and dried under vacuum. The product was obtained a white solid (25 $\mathrm{mg}, 0.085 \mathrm{mmol}, 87 \%) .{ }^{1} \mathrm{H}$ NMR $\left(500 \mathrm{MHz}, \mathrm{D}_{2} \mathrm{O}\right) \delta 7.23(\mathrm{dt}, \mathrm{J}=8.6,6.8,3 \mathrm{H}), 7.17-$ $7.04(\mathrm{~m}, 2 \mathrm{H}), 4.90-4.81(\mathrm{~m}, 1 \mathrm{H}), 3.72(\mathrm{~s}, 2 \mathrm{H}), 3.43-3.36(\mathrm{~m}, 4 \mathrm{H}), 3.25-3.00(\mathrm{~m}$, 4H). 13C NMR (126 MHz, $\left.D_{2} \mathrm{O}\right) \delta 154.29,135.35,129.04,128.79,127.27,66.52,60.75$, 46.11, 42.45, 35.40. MS (ESI): $m / z(\%): 257.21(100)\left[M^{-}-H\right]$.

(1H-tetrazol-5-yl)methanamine hydrochloride (9). To a suspension of $1 \mathrm{H}$-tetrazole ${ }^{\mathrm{HCl}}$
$\mathrm{H}_{2} \mathrm{~N}^{\mathrm{N}=\mathrm{N}}$
$\mathrm{N} H$ $6 \mathrm{~h}(168 \mathrm{mg}, 0.49 \mathrm{mmol})$ in 1,4-dioxane $(2.5 \mathrm{~mL})$ was added $4.0 \mathrm{M} \mathrm{HCl}$ in dioxane $(250 \mu \mathrm{L}, 1 \mathrm{mmol})$. The reaction was stirred at room temperature for $5 \mathrm{~min}$. The reaction was purified by filtration through silica. The impurities were eluted with EtOAc/Petroleum ether $(1: 1,30 \mathrm{~mL})$. The product was eluted with $\mathrm{MeOH} / \mathrm{CH}_{2} \mathrm{Cl}_{2}(3: 1,30 \mathrm{~mL})$, and concentrated in vacuo. The product was obtained as a white solid (97 mg, $0.45 \mathrm{mmol}, 92 \%) .{ }^{1} \mathrm{H} \mathrm{NMR}(500 \mathrm{MHz}$, $\left.\mathrm{D}_{2} \mathrm{O}\right) \delta 4.36 .{ }^{13} \mathrm{C}$ NMR (126 MHz, DMSO) $\delta ~ 34.71$. MS (ESI): $m / z(\%): 98.17(100)\left[M^{-}-\mathrm{H}\right]$. 
${ }^{1} \mathrm{H}$ NMR and ${ }^{13} \mathrm{C}$ NMR Spectra 
N-(2-cyanoethyl)formamide (4)

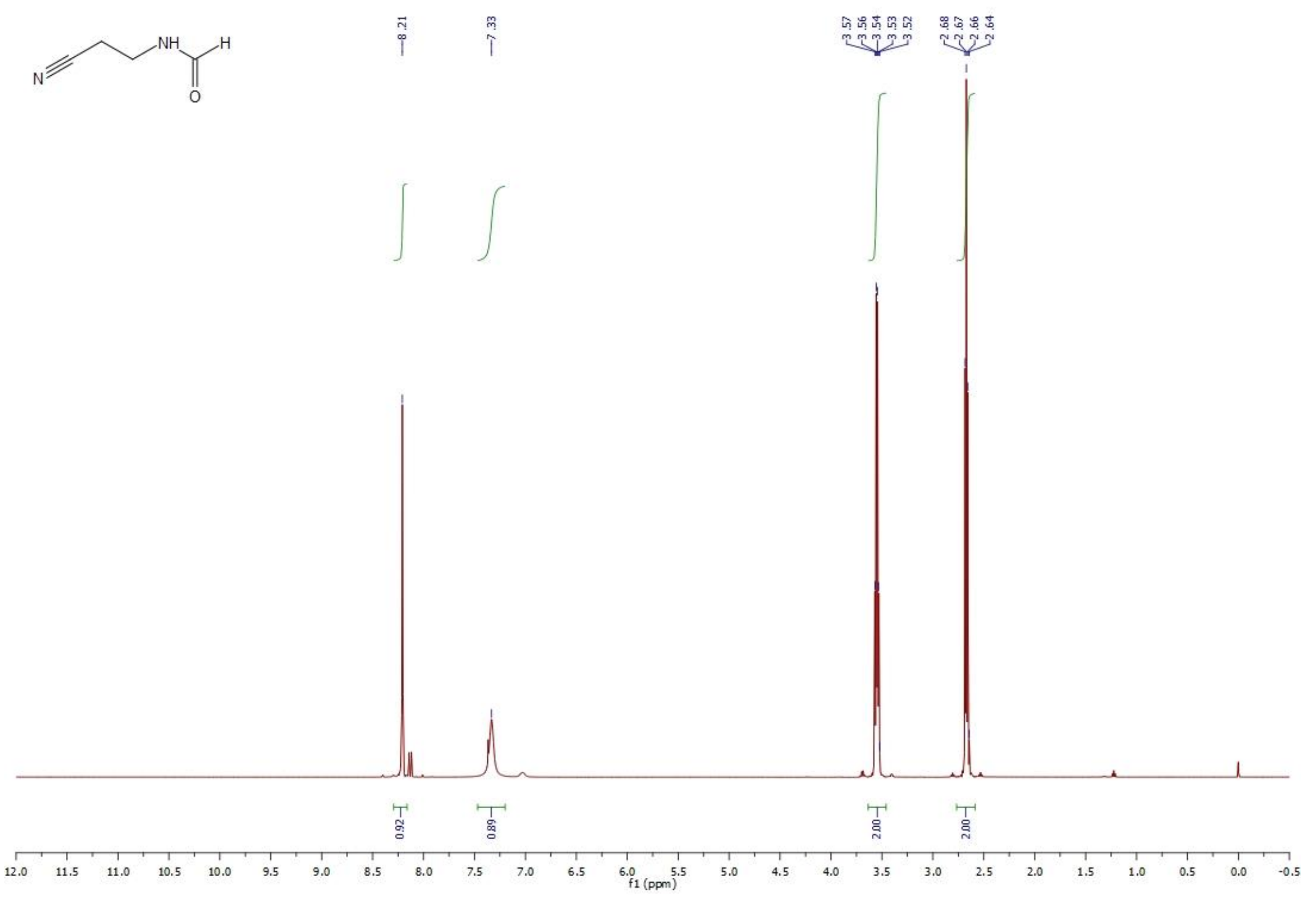



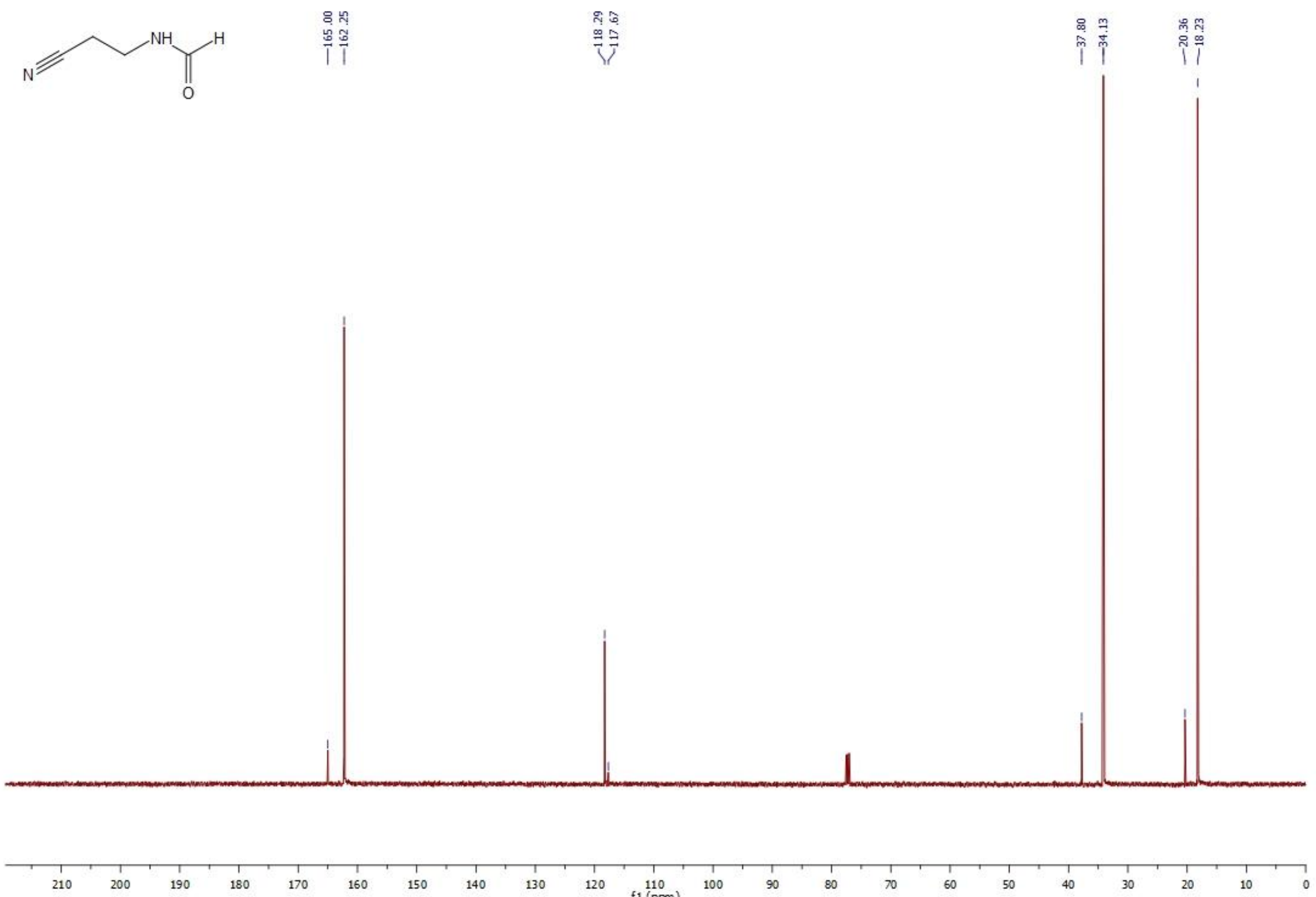
3-isocyanopropanenitrile (2)

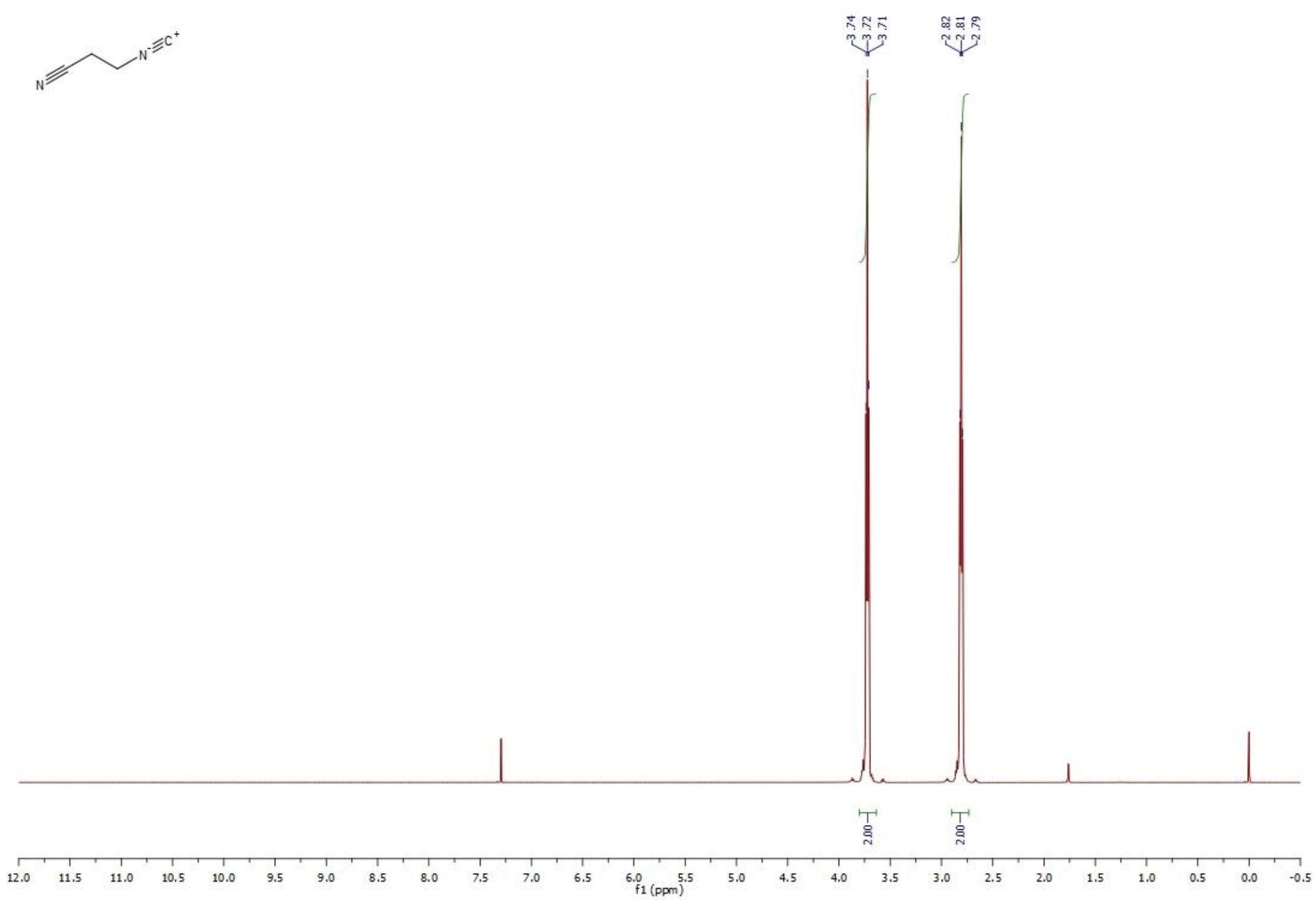




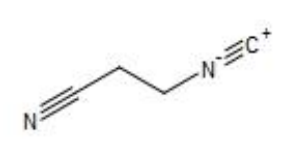


3-(5-((butylamino)(4-isopropylphenyl)methyl)-1H-tetrazol-1-yl)propanenitrile (5a)
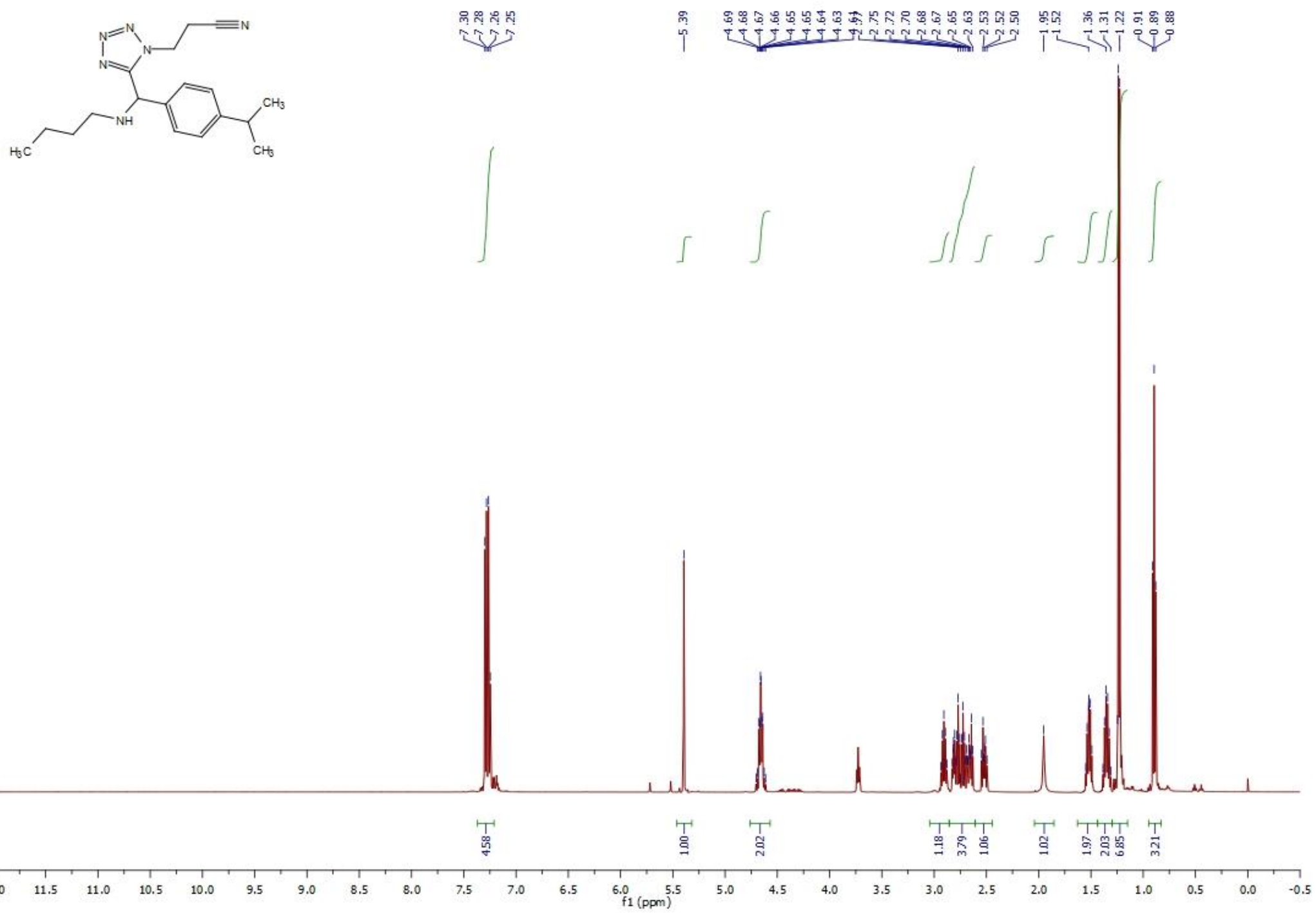

S18 

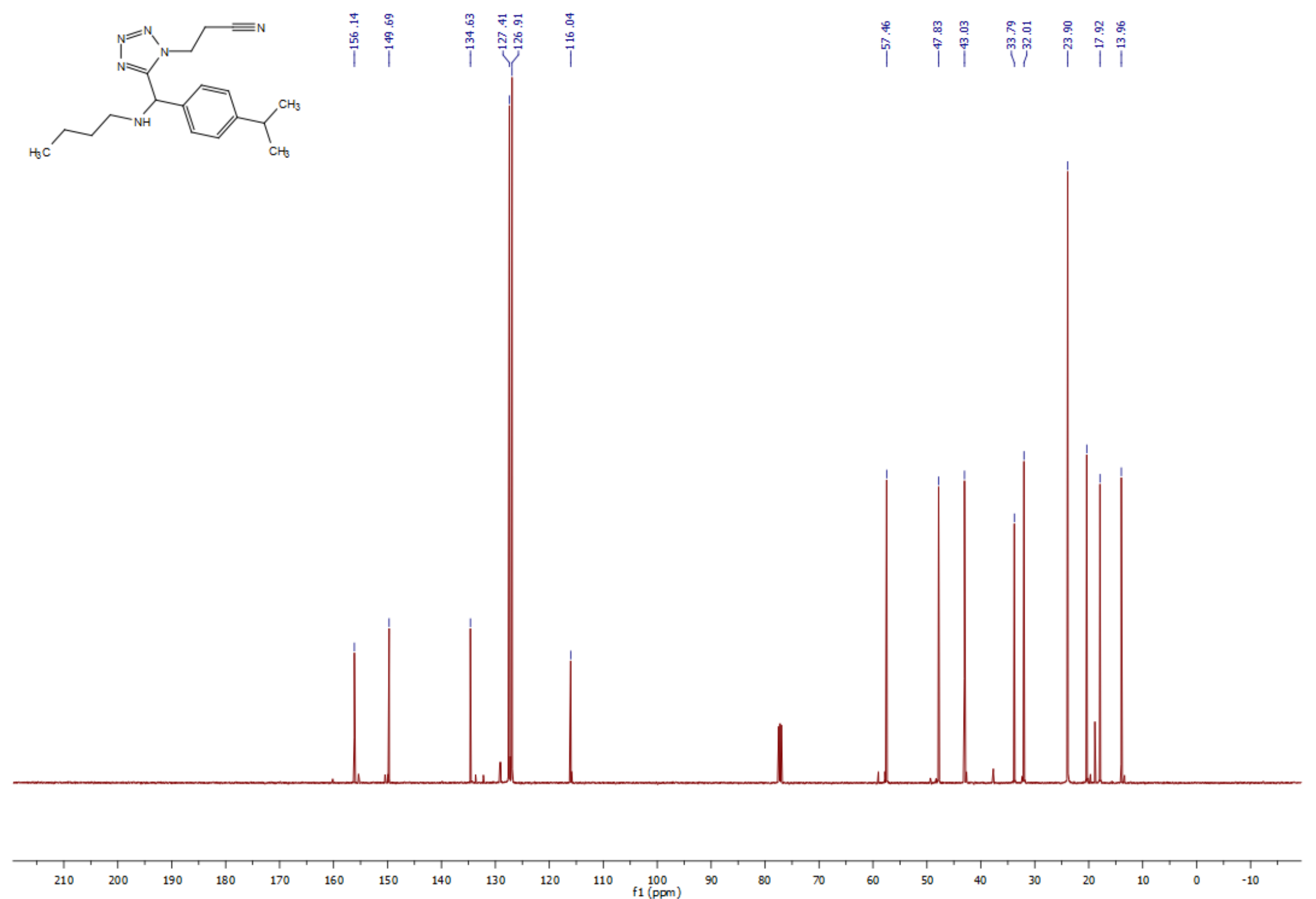
3-(5-(cyclohexyl(mesitylamino)methyl)-1H-tetrazol1-yl)propanenitrile (5b)

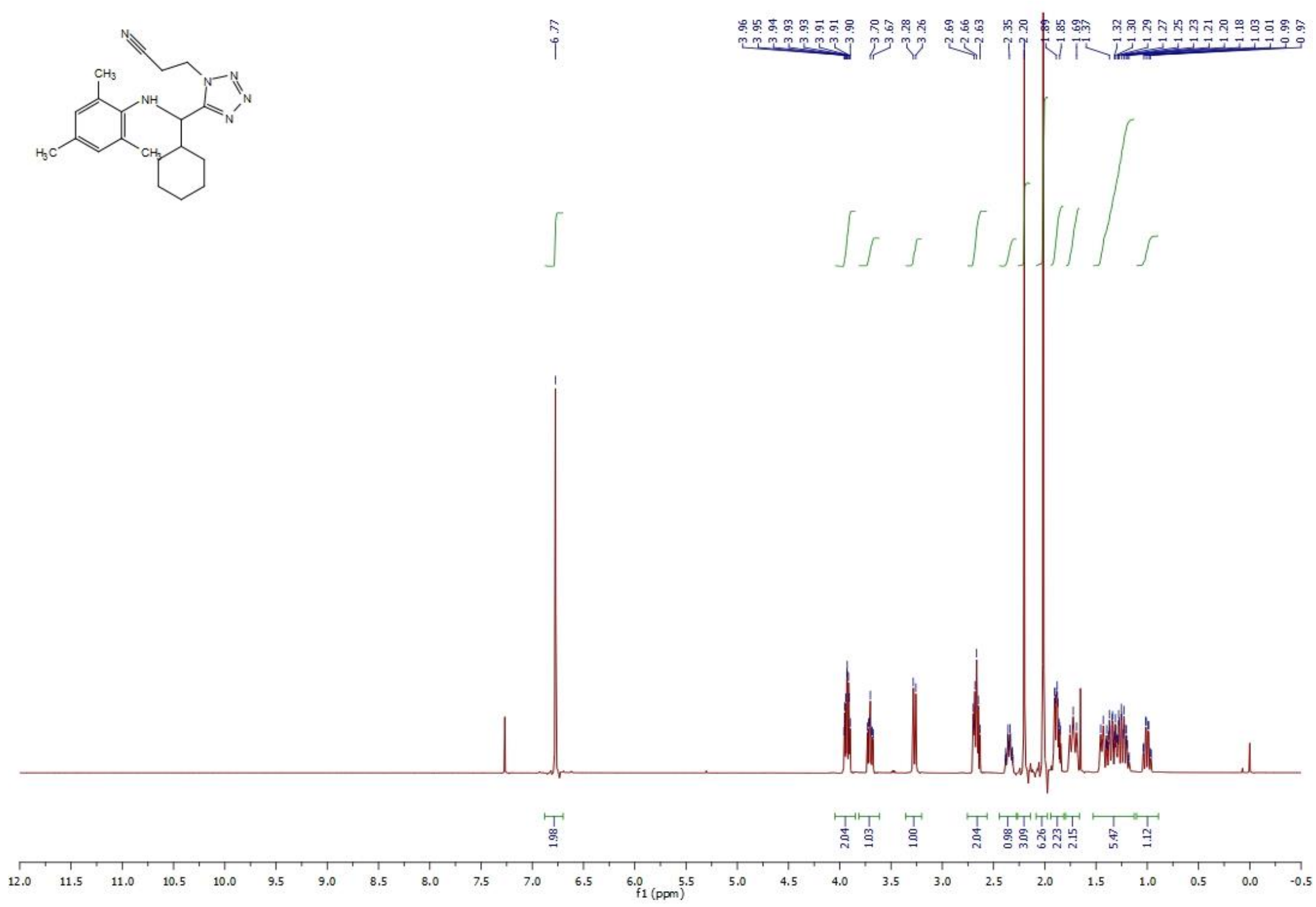




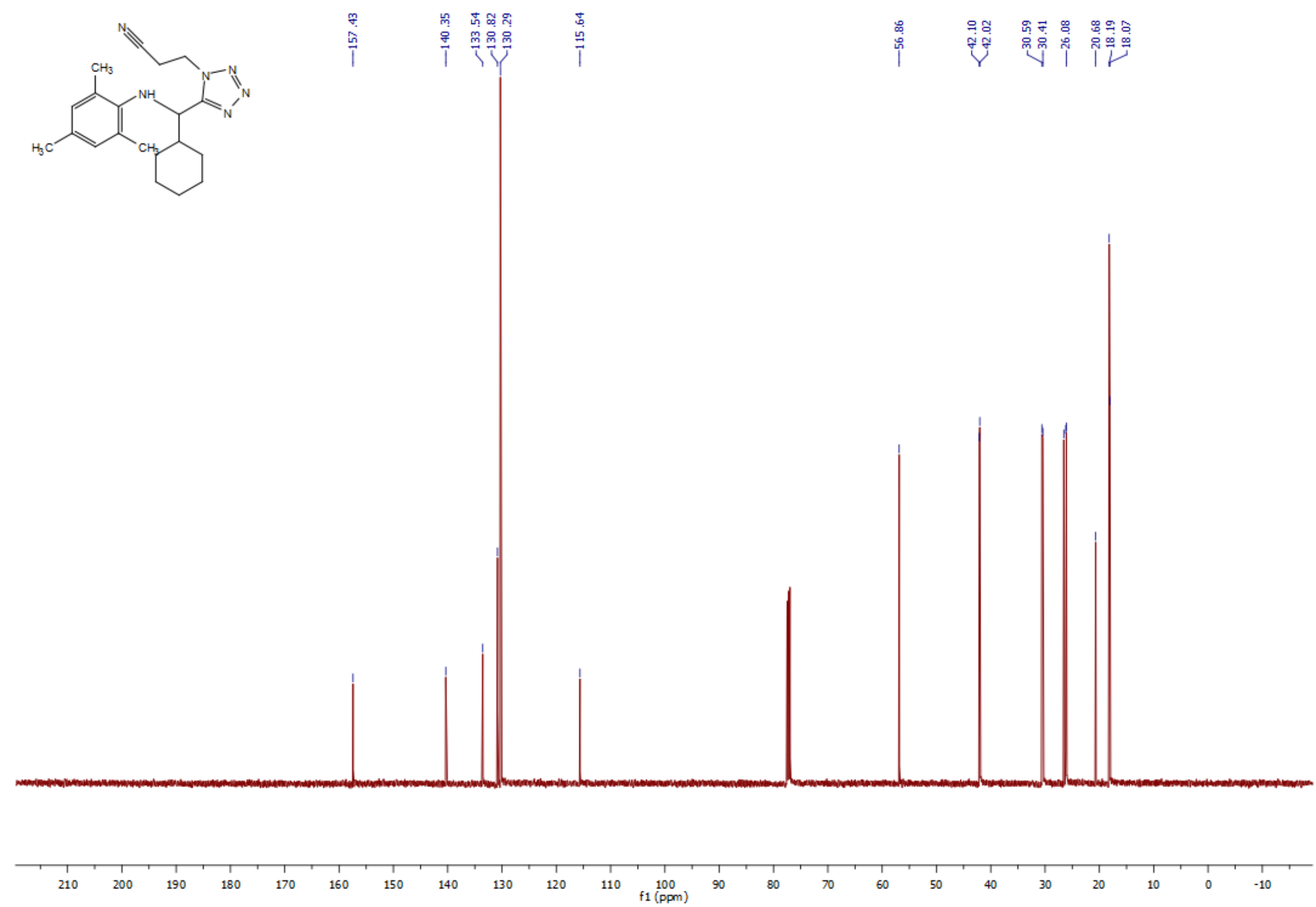


3-(5-(1-(phenylamino)cyclohexyl)-1H-tetrazol-1-yl)propanenitrile (5c)

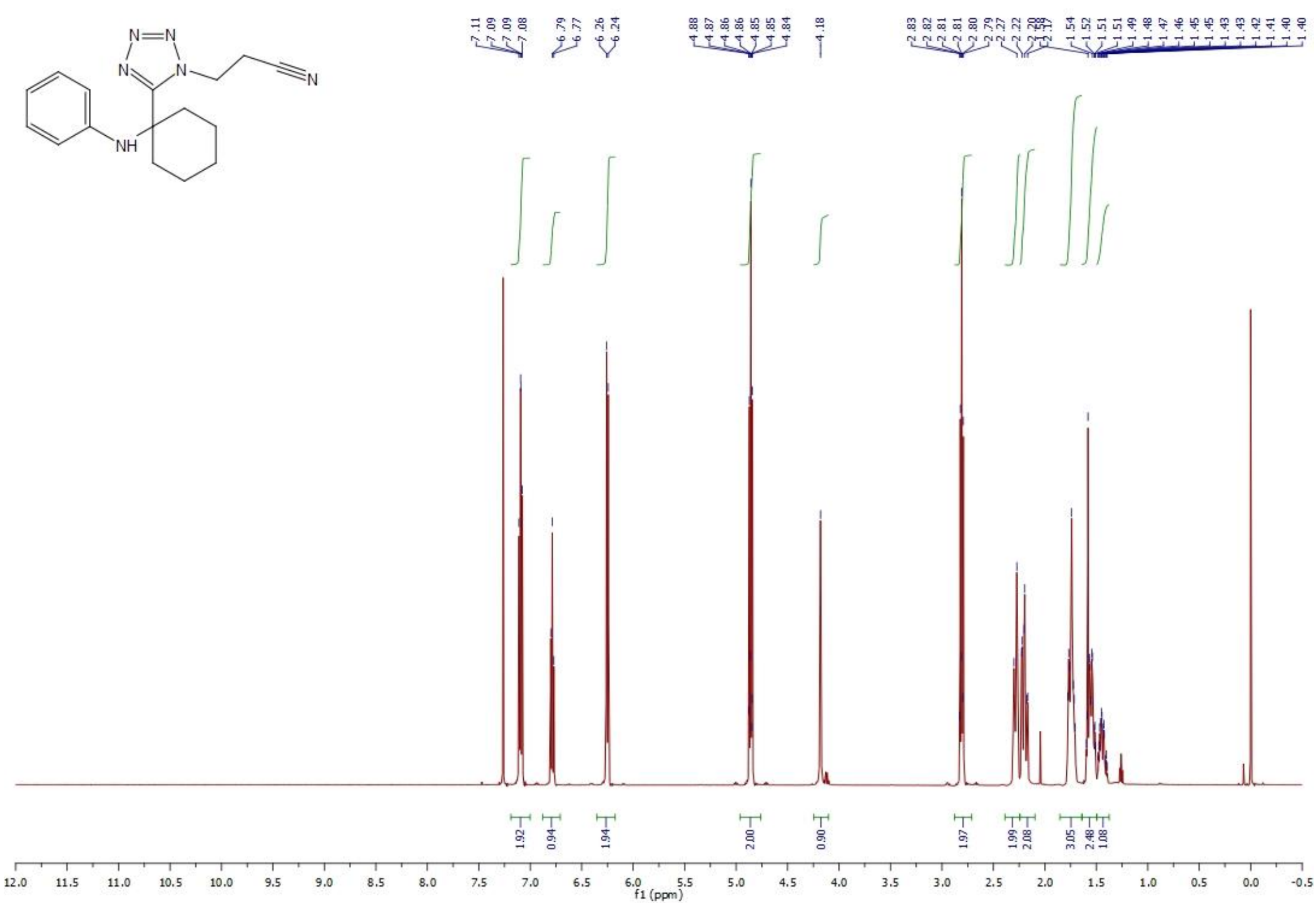




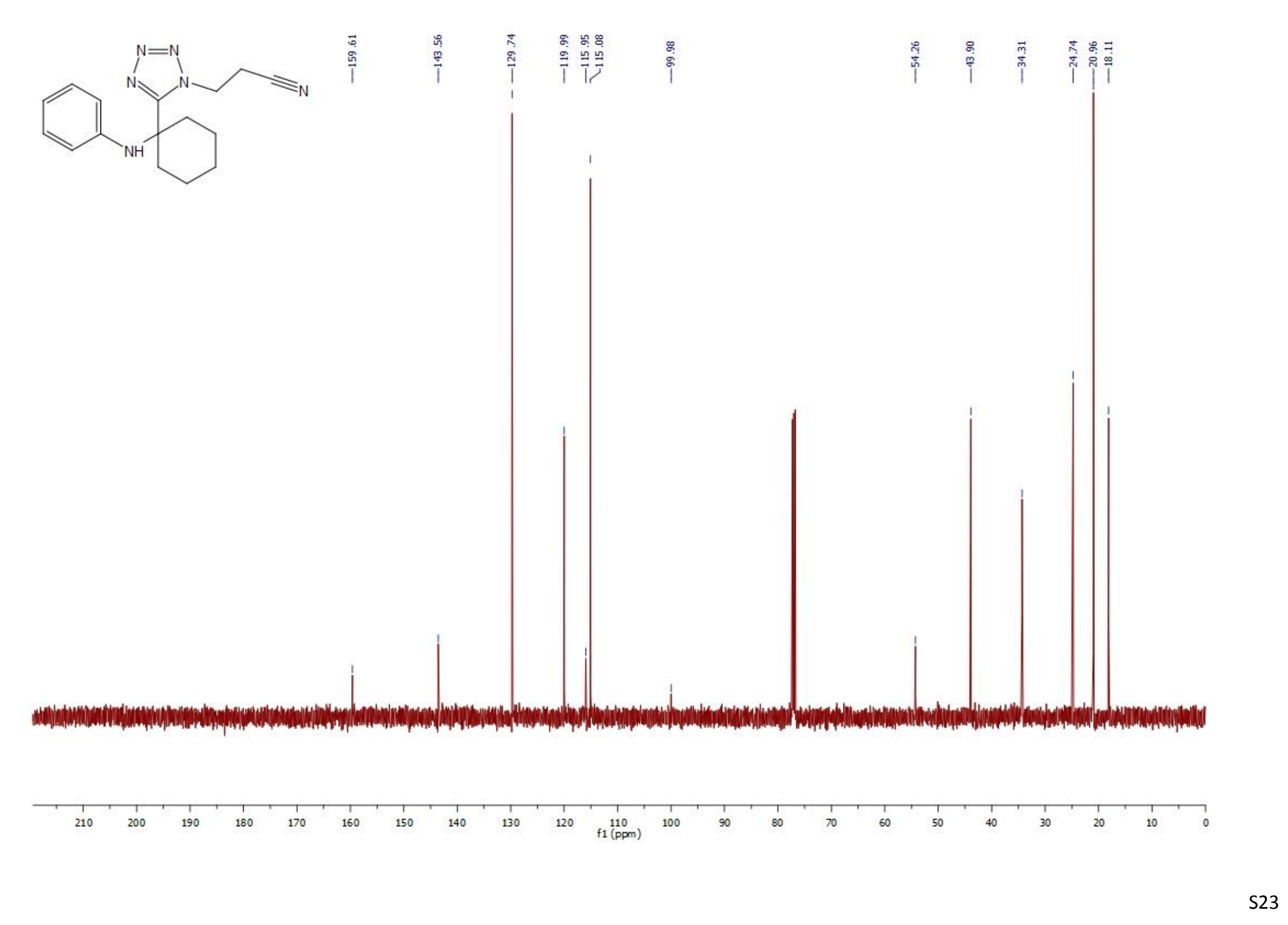


4-((1-(1-(2-cyanoethyl)-1H-tetrazol-5-yl)-2-methylpropyl)amino)benzonitrile (5d)

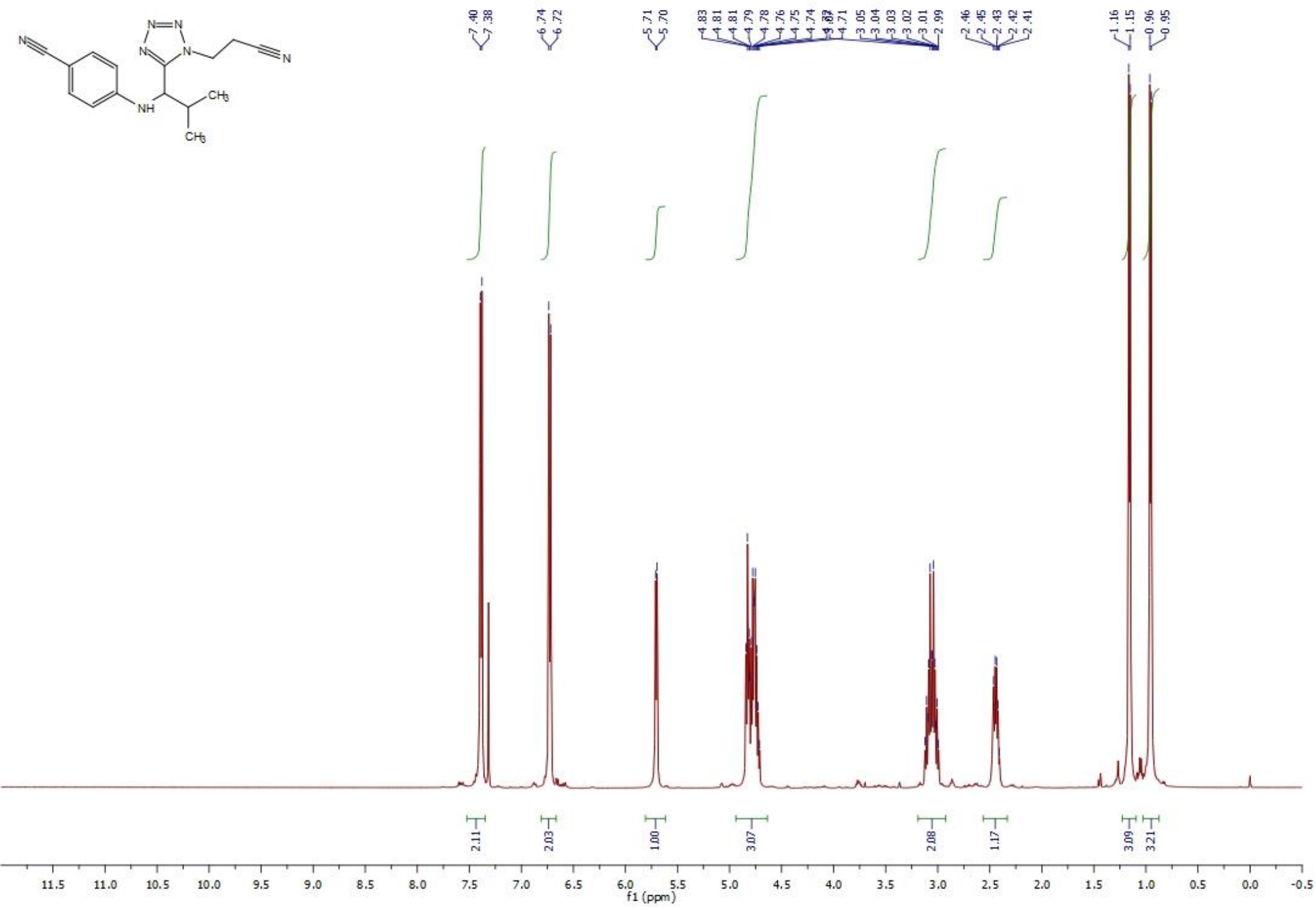


笽 
3-(5-(benzo[d][1,3]dioxol-5-yl(pyrrolidin-1-yl)methyl)-1H-tetrazol-1-yl)propanenitrile (5e)

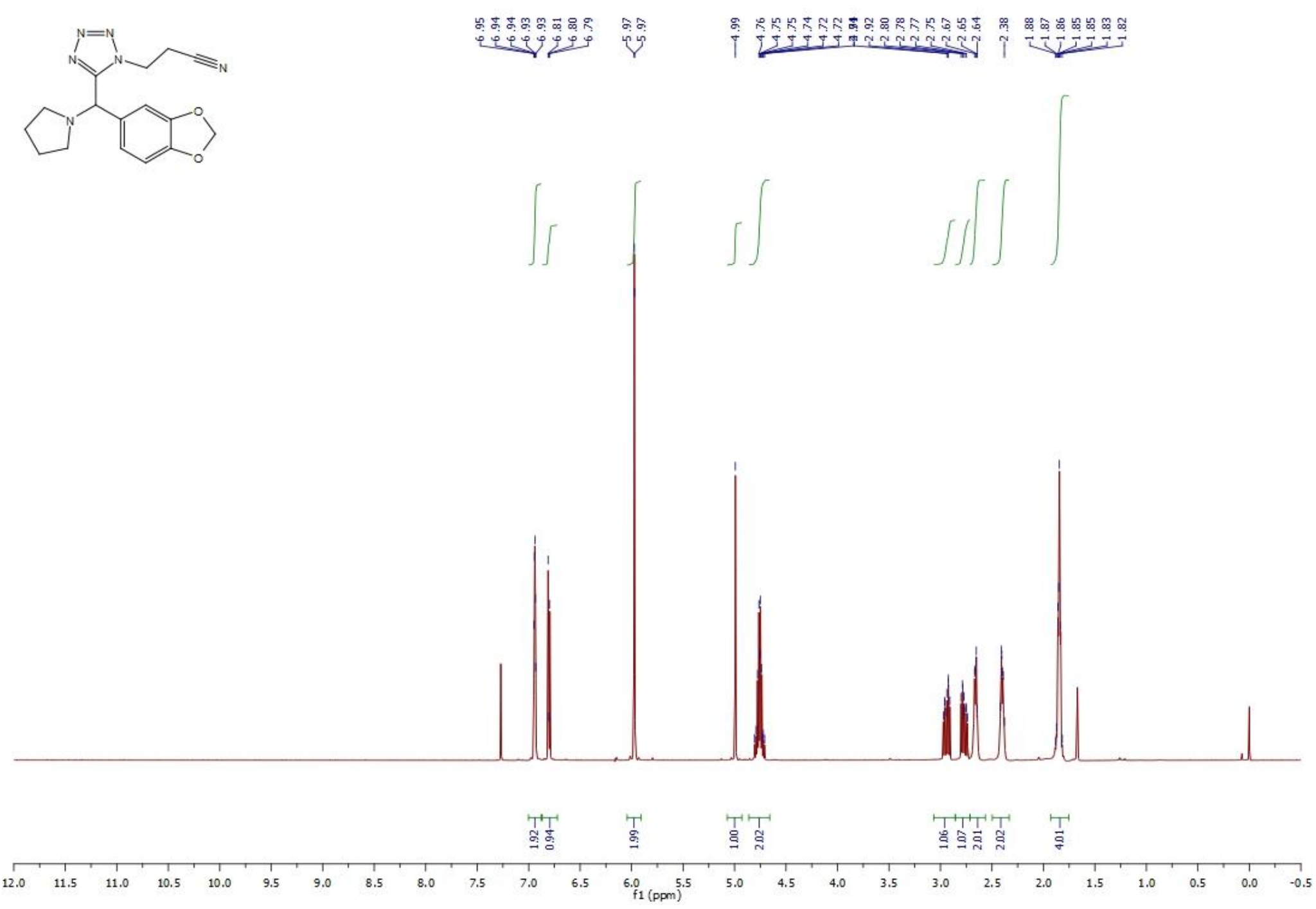



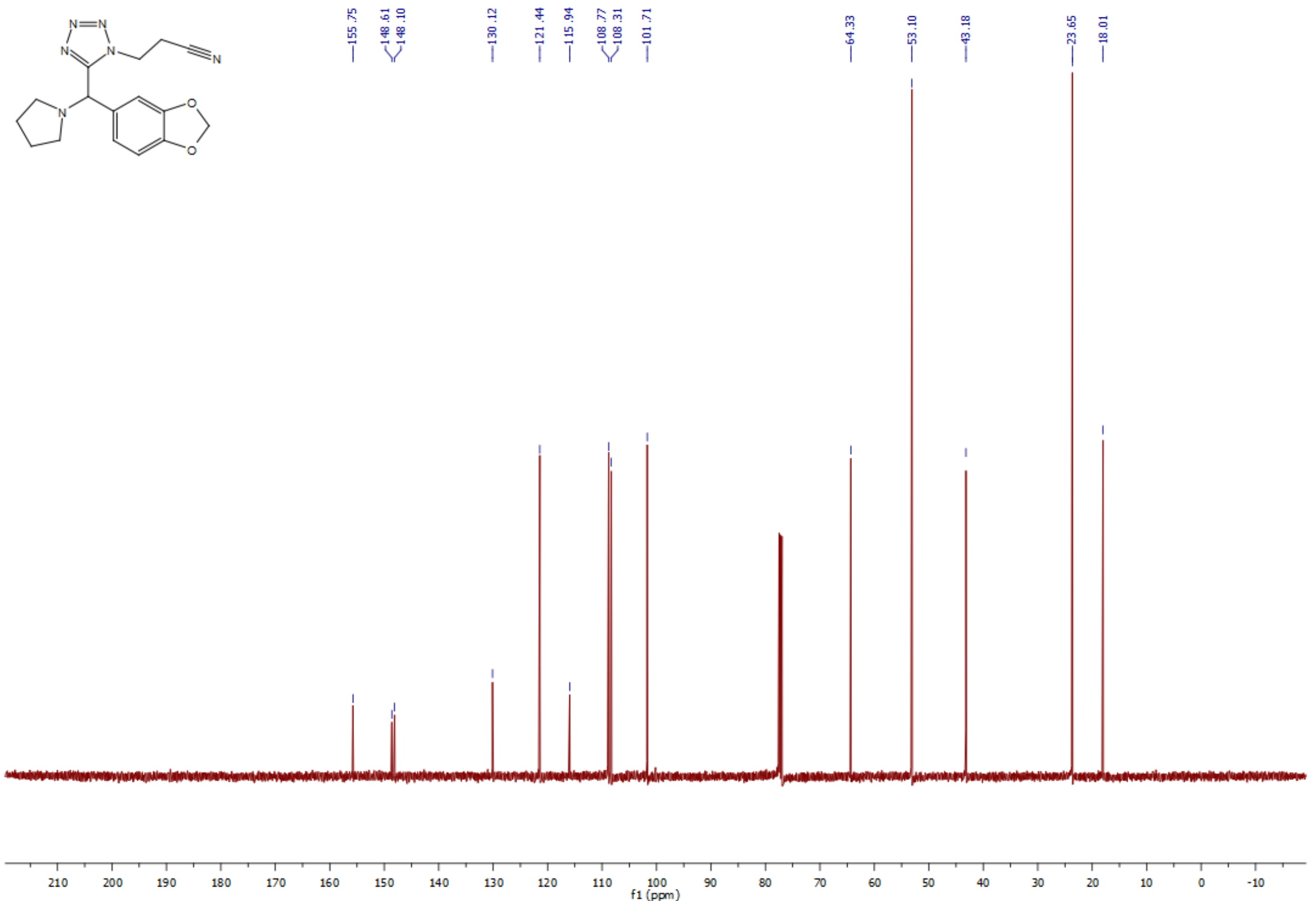
3-(5-(morpholino(naphthalen-1-yl)methyl)-1H-tetrazol-1-yl)propanenitrile (5f)

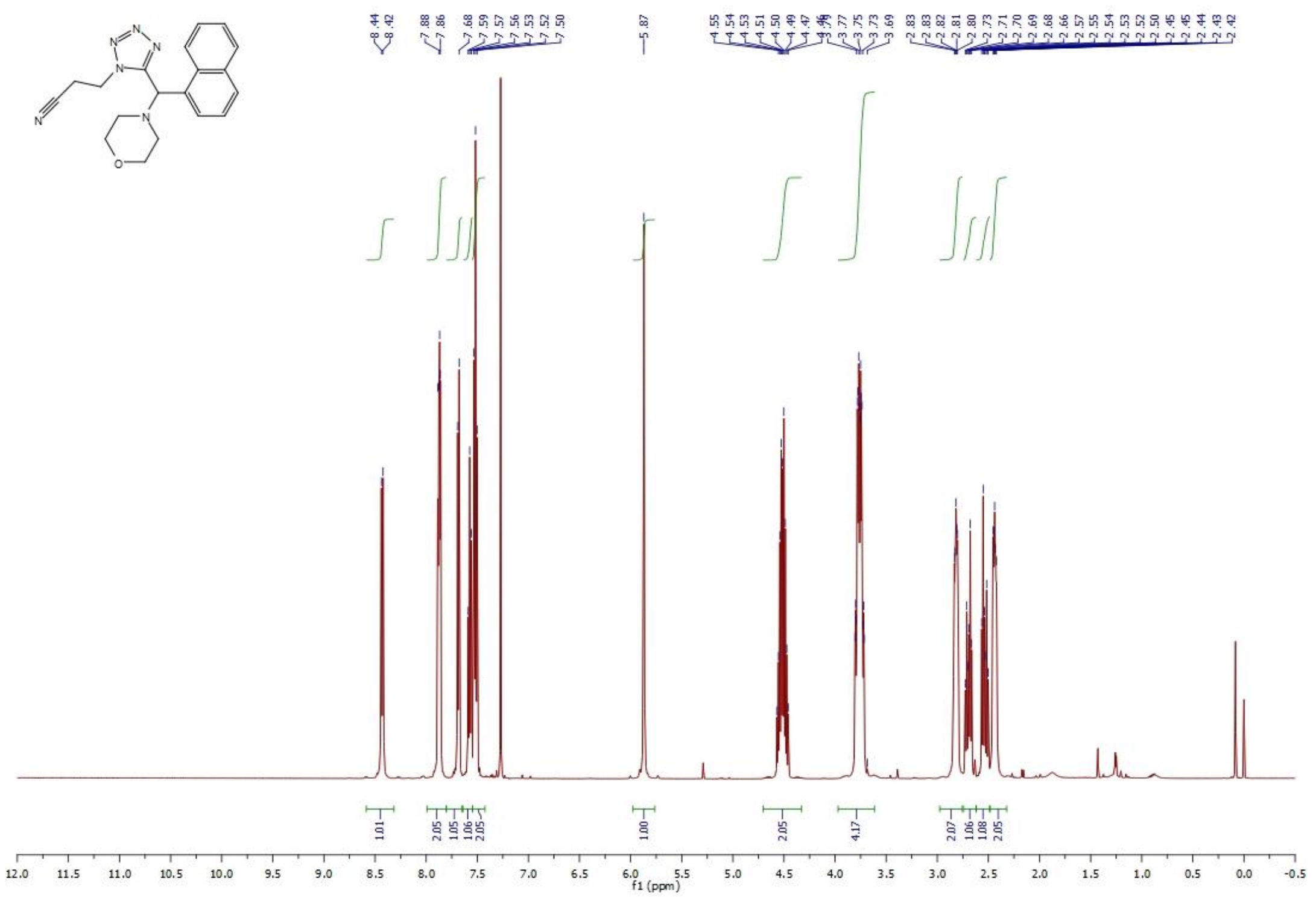



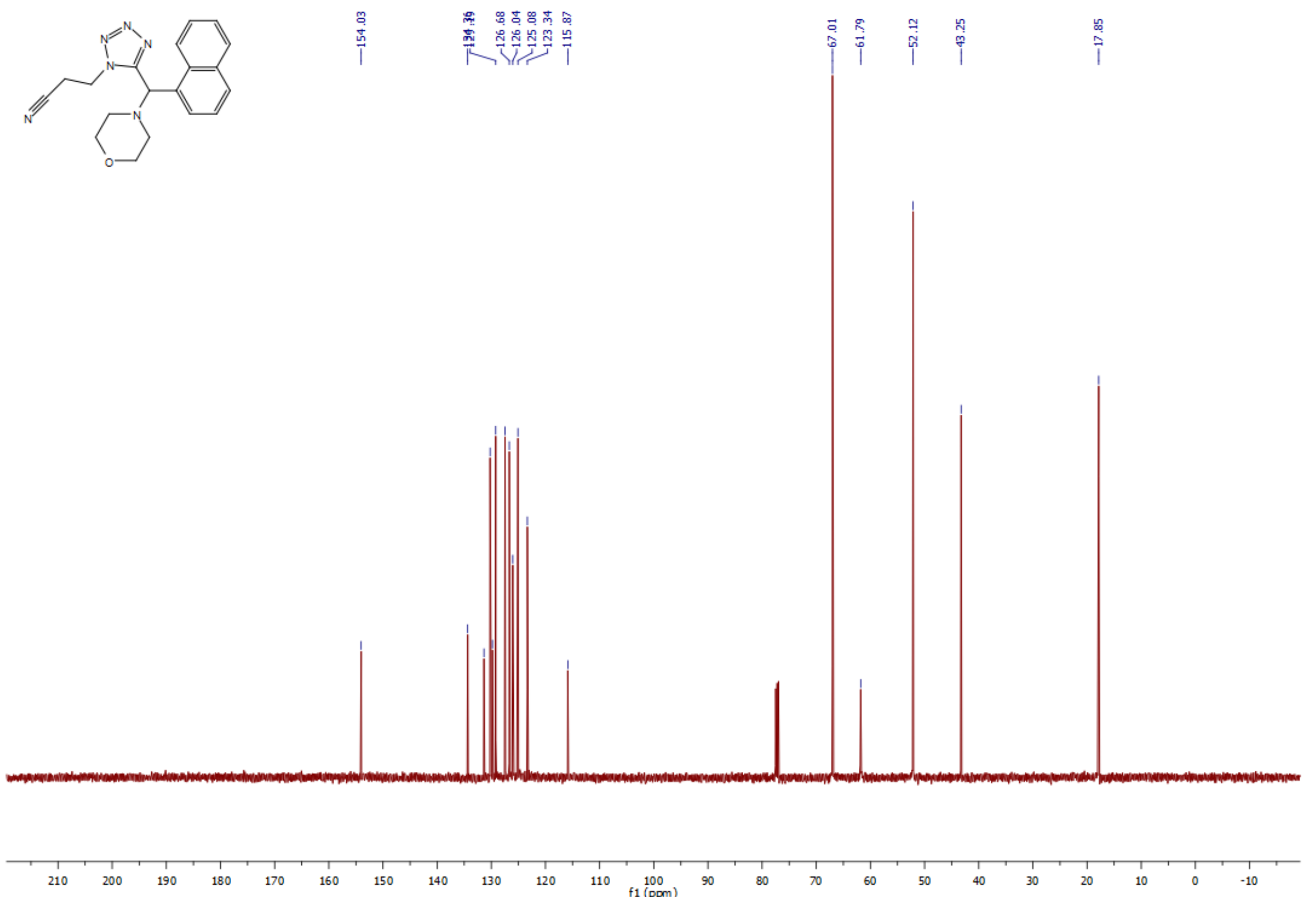
tert-butyl 4-(1-(1-(2-cyanoethyl)-1H-tetrazol-5-yl)-2-phenylethyl)piperazine-1-carboxylate (5g)

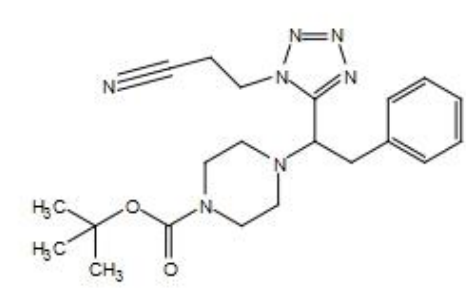

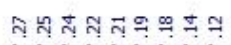
20isisis

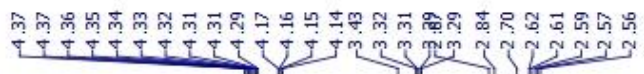
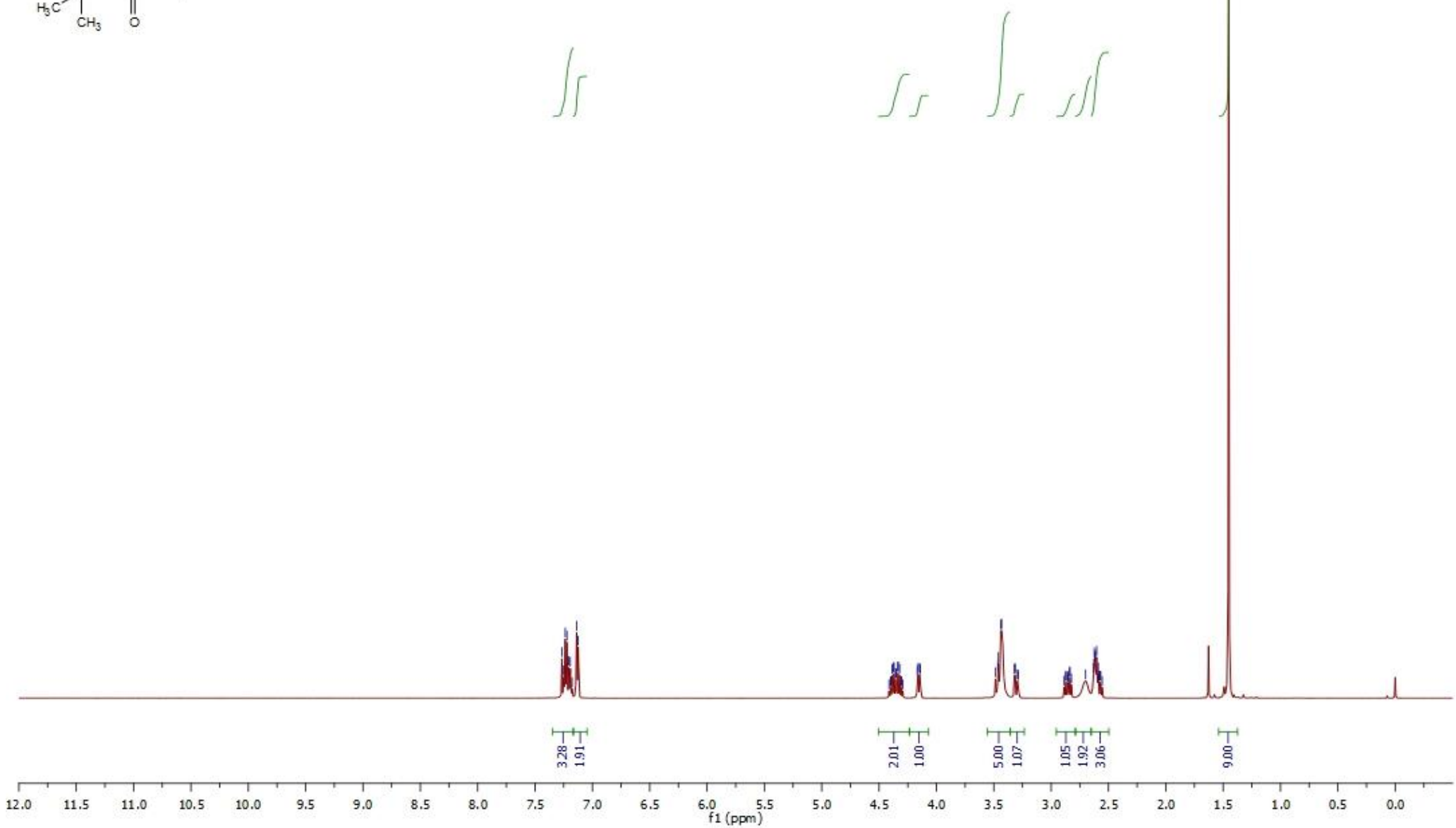

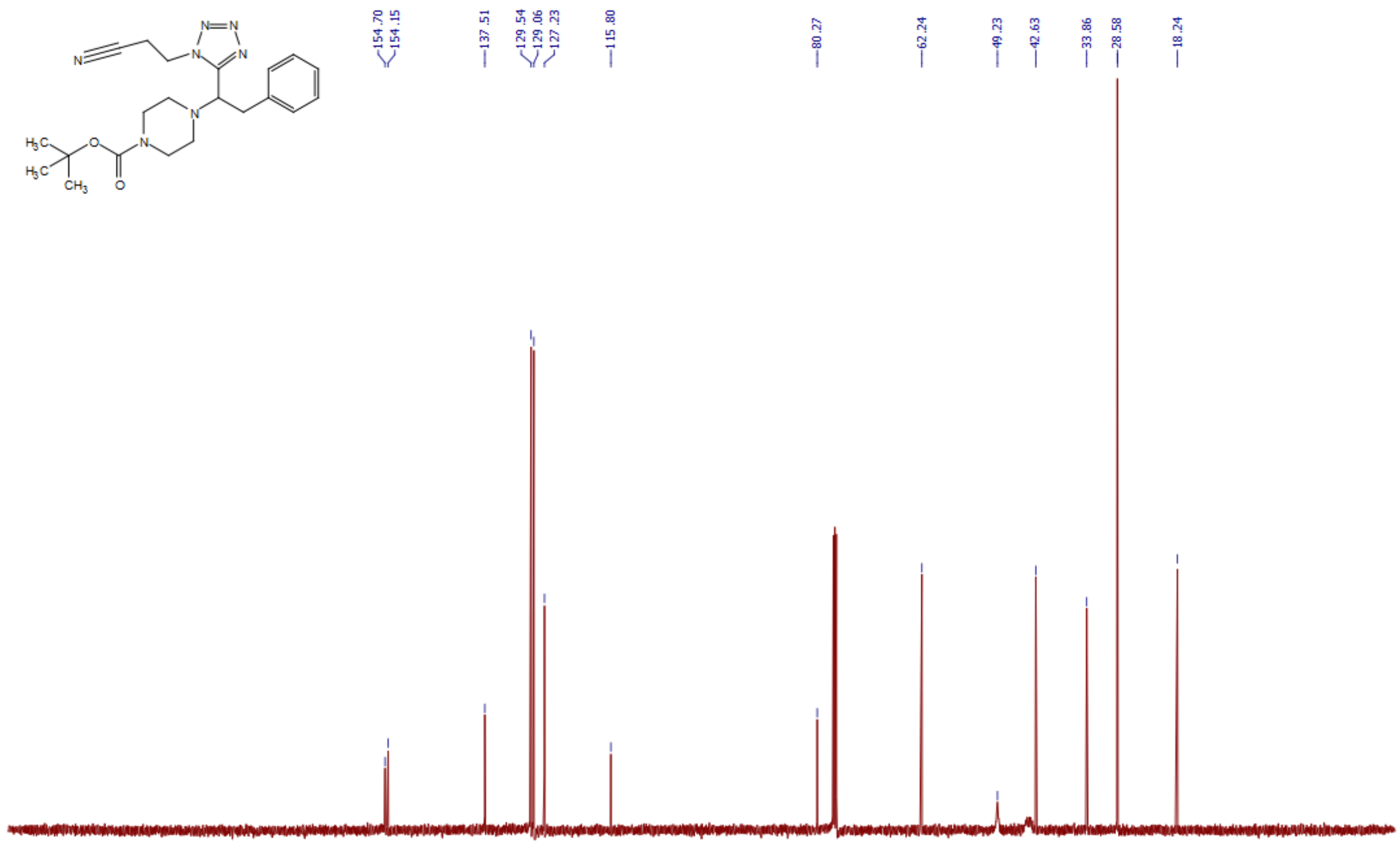
3-(5-((tritylamino)methyl)-1H-tetrazol-1-yl)propanenitrile (5h)

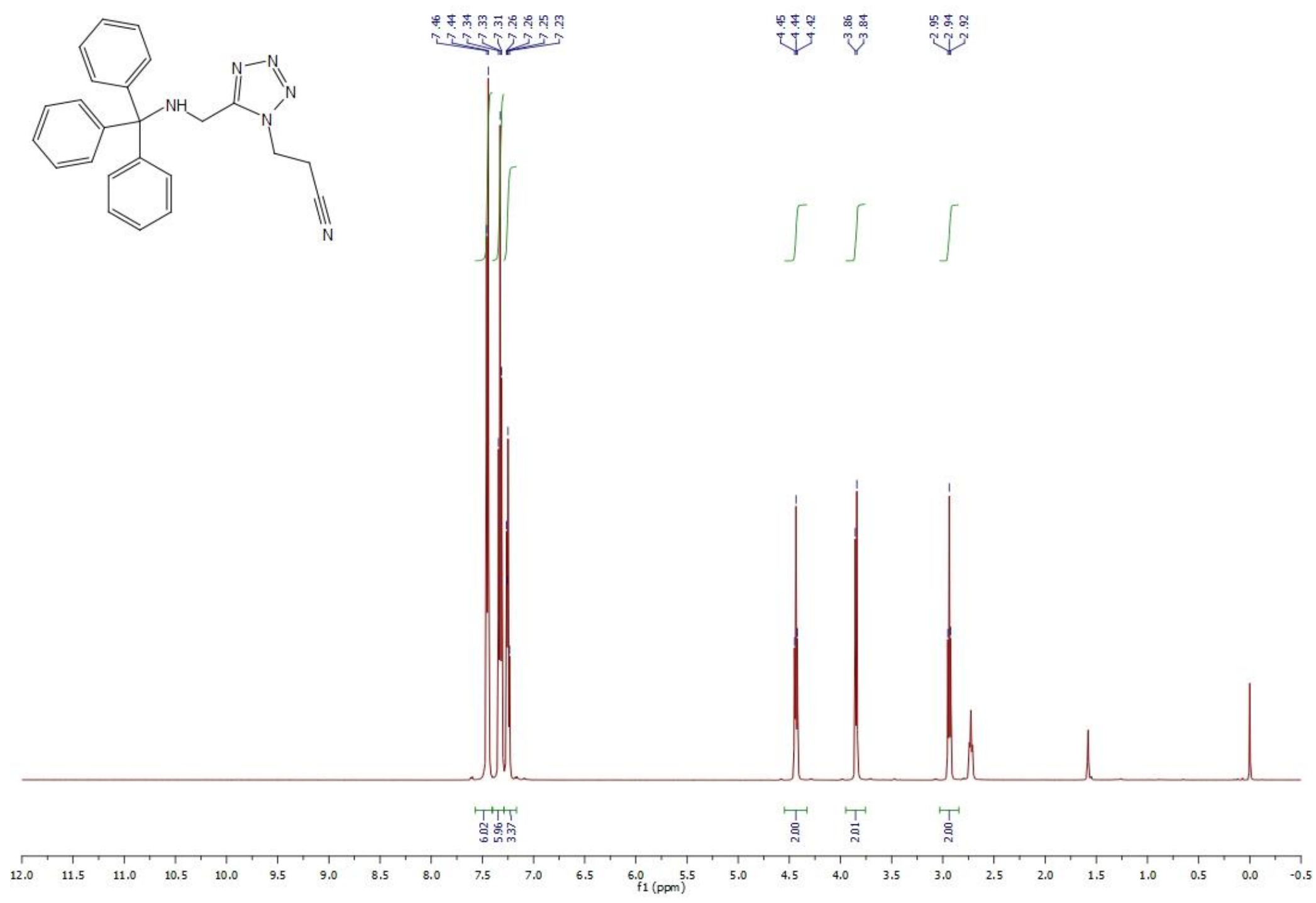




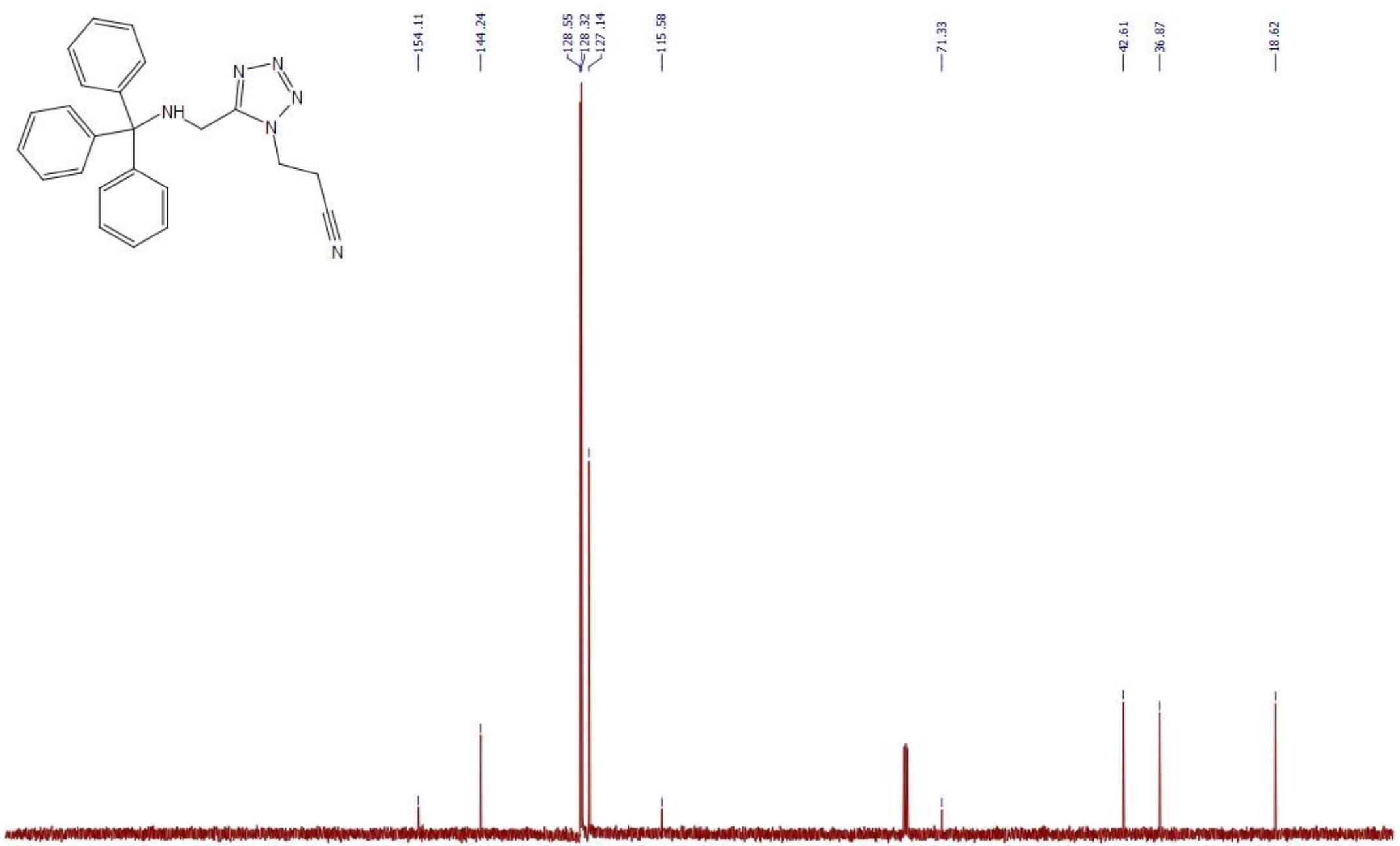


3-(5-(2-((thiophen-2-ylmethyl)amino)propan-2-yl)-1H-tetrazol-1-yl)propanenitrile (5i)

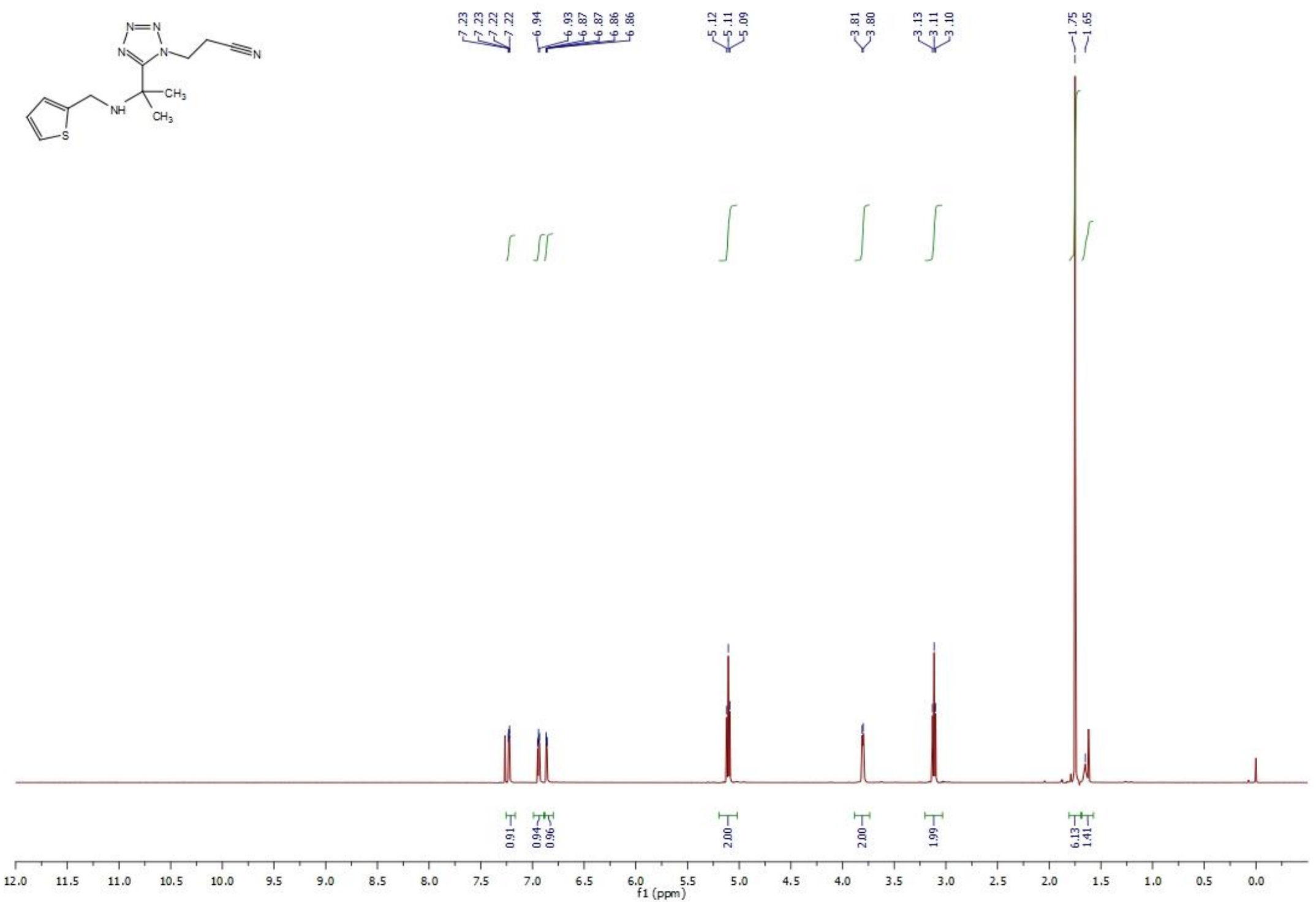



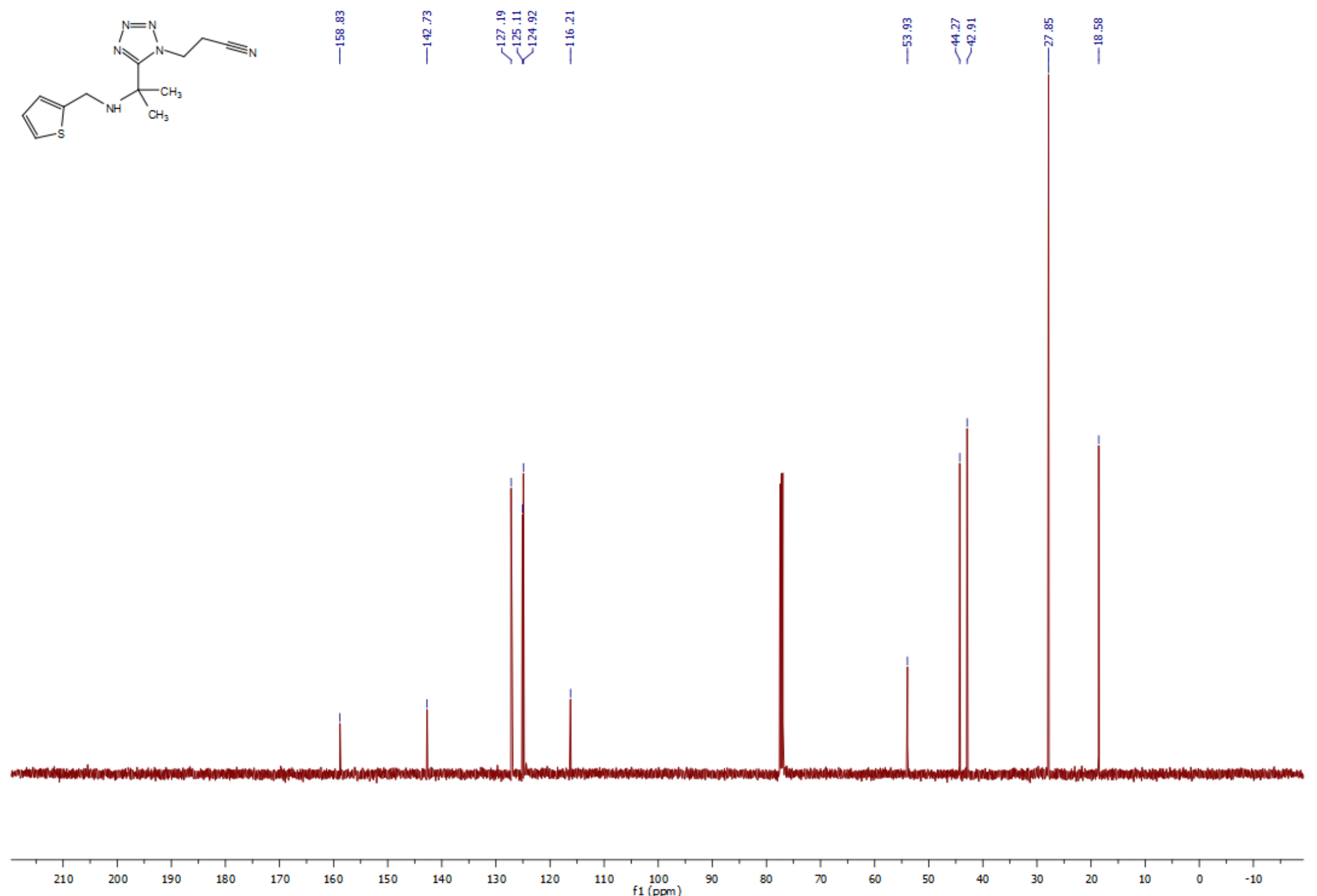
3-(5-(benzo[b]thiophen-2-yl(tert-butylamino)methyl)-1H-tetrazol-1-yl)propanenitrile (5j)

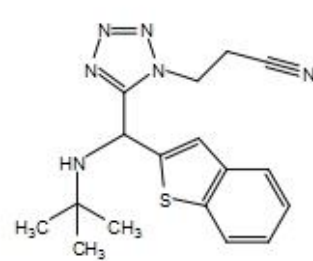

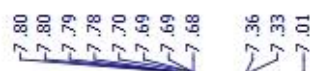

Yับ
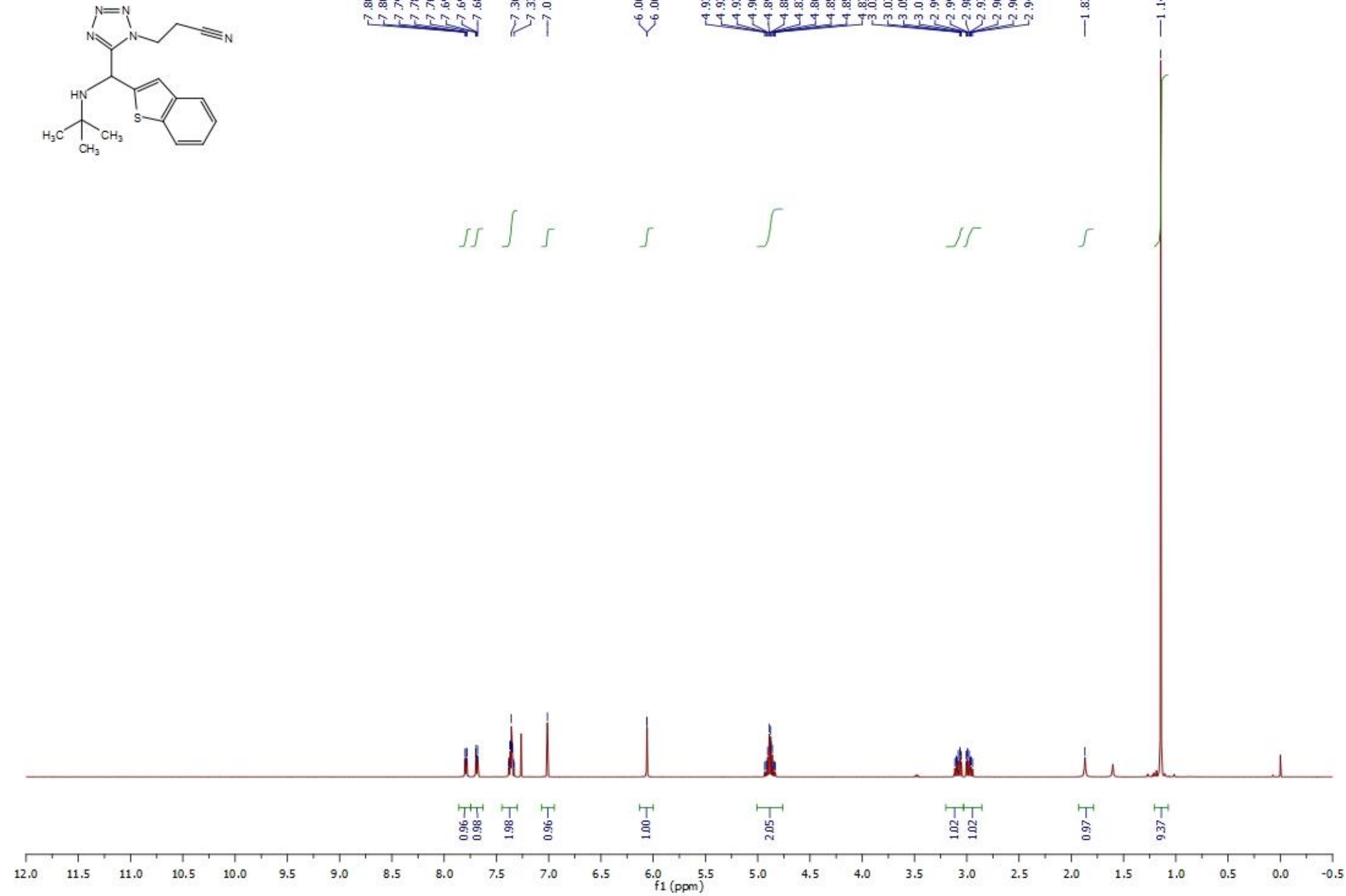

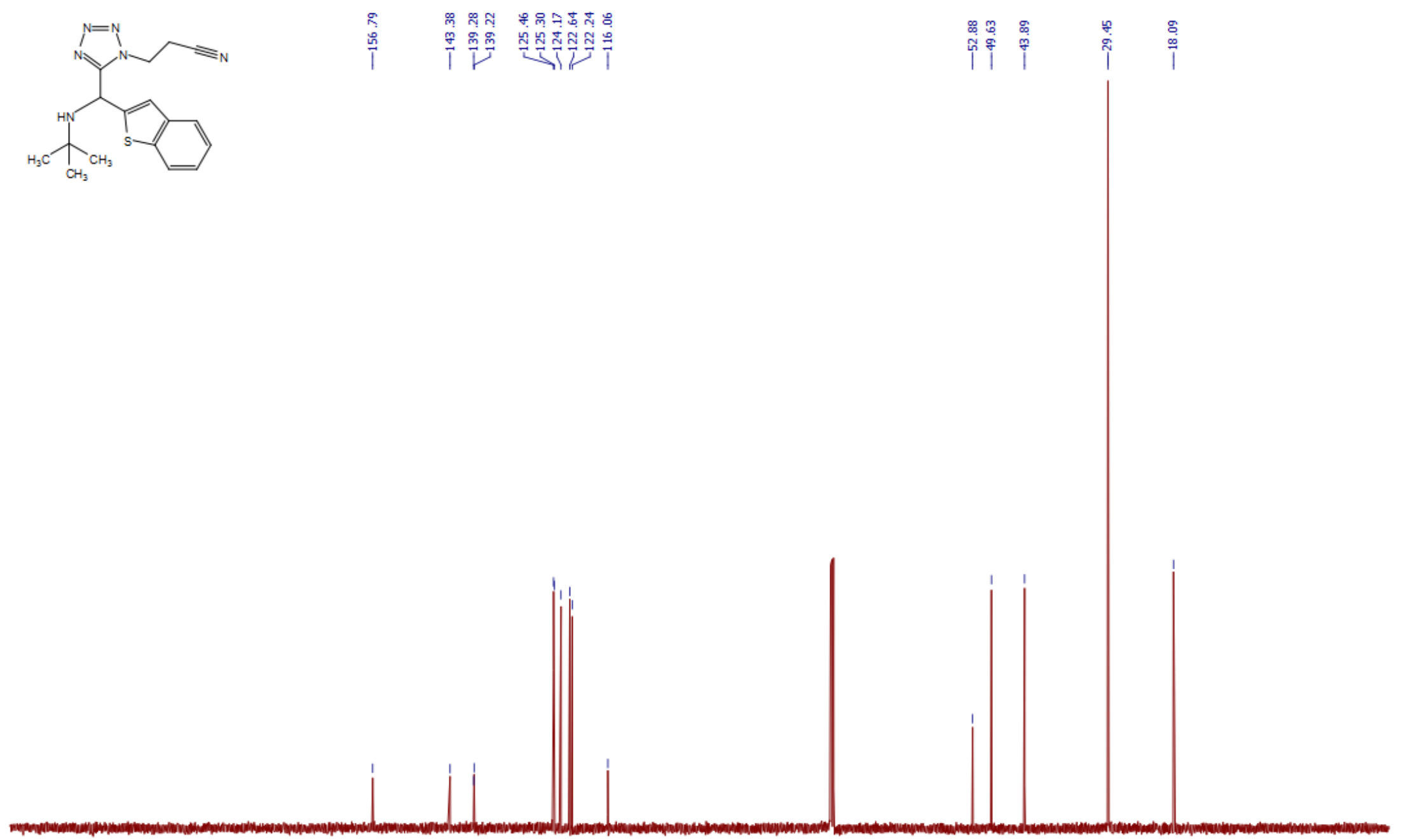
3-(5-(((2-hydroxyethyl)amino)(pyridin-3-yl)methyl)-1H-tetrazol-1-yl)propanenitrile (5k)
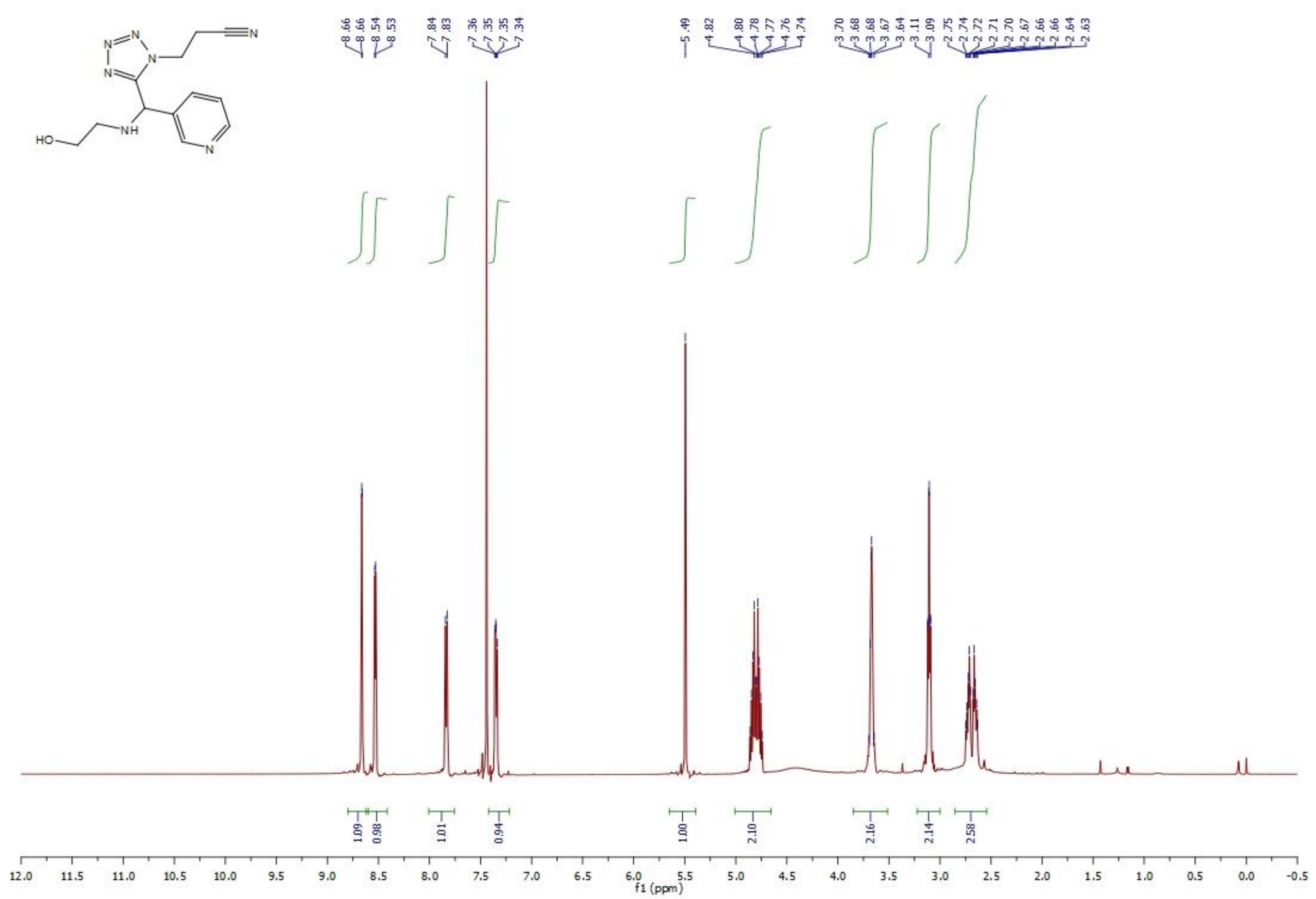

S38 

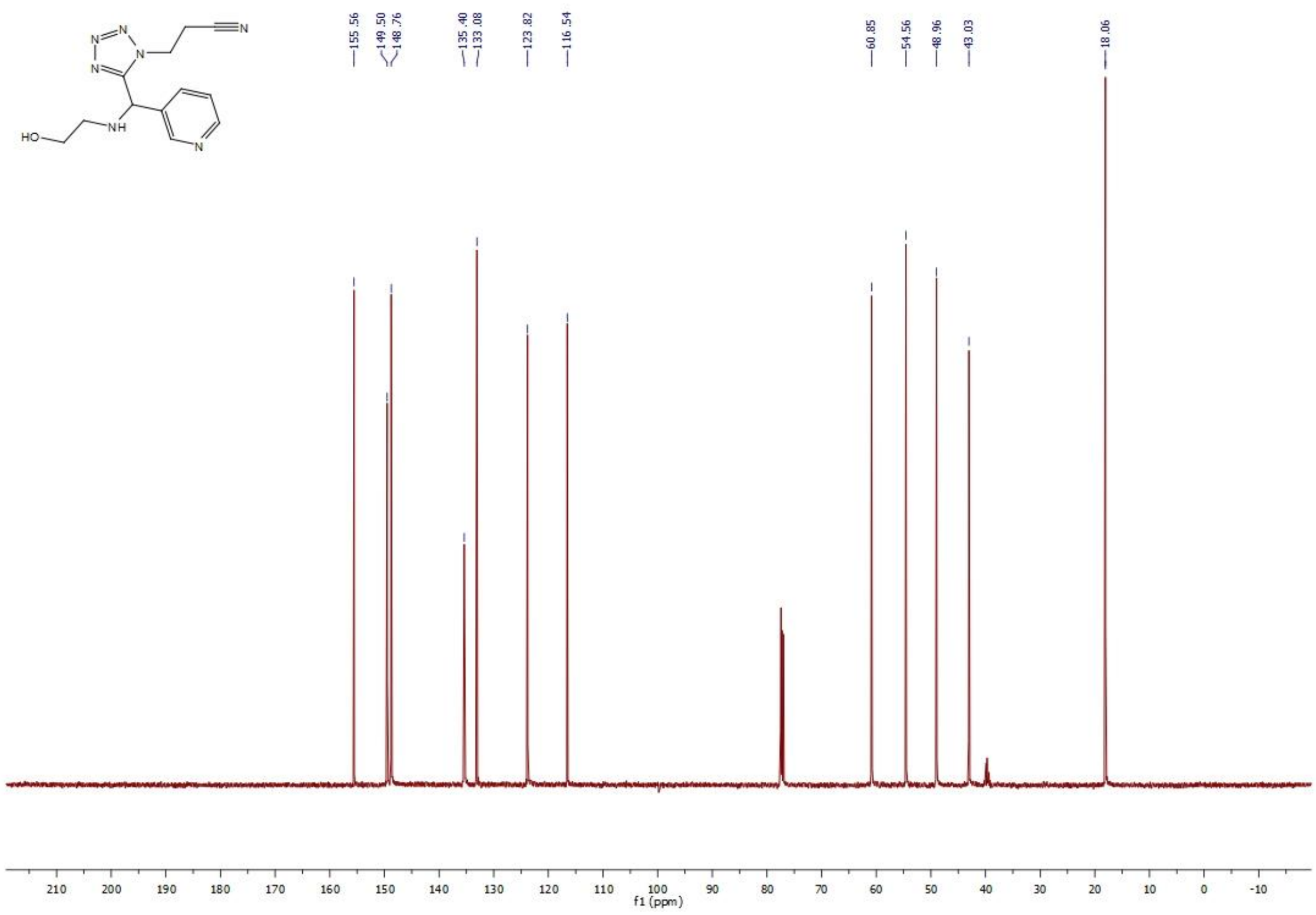

S39 
N-((4-isopropylphenyl)(1H-tetrazol-5-yl)methyl)butan-1-amine (6a)

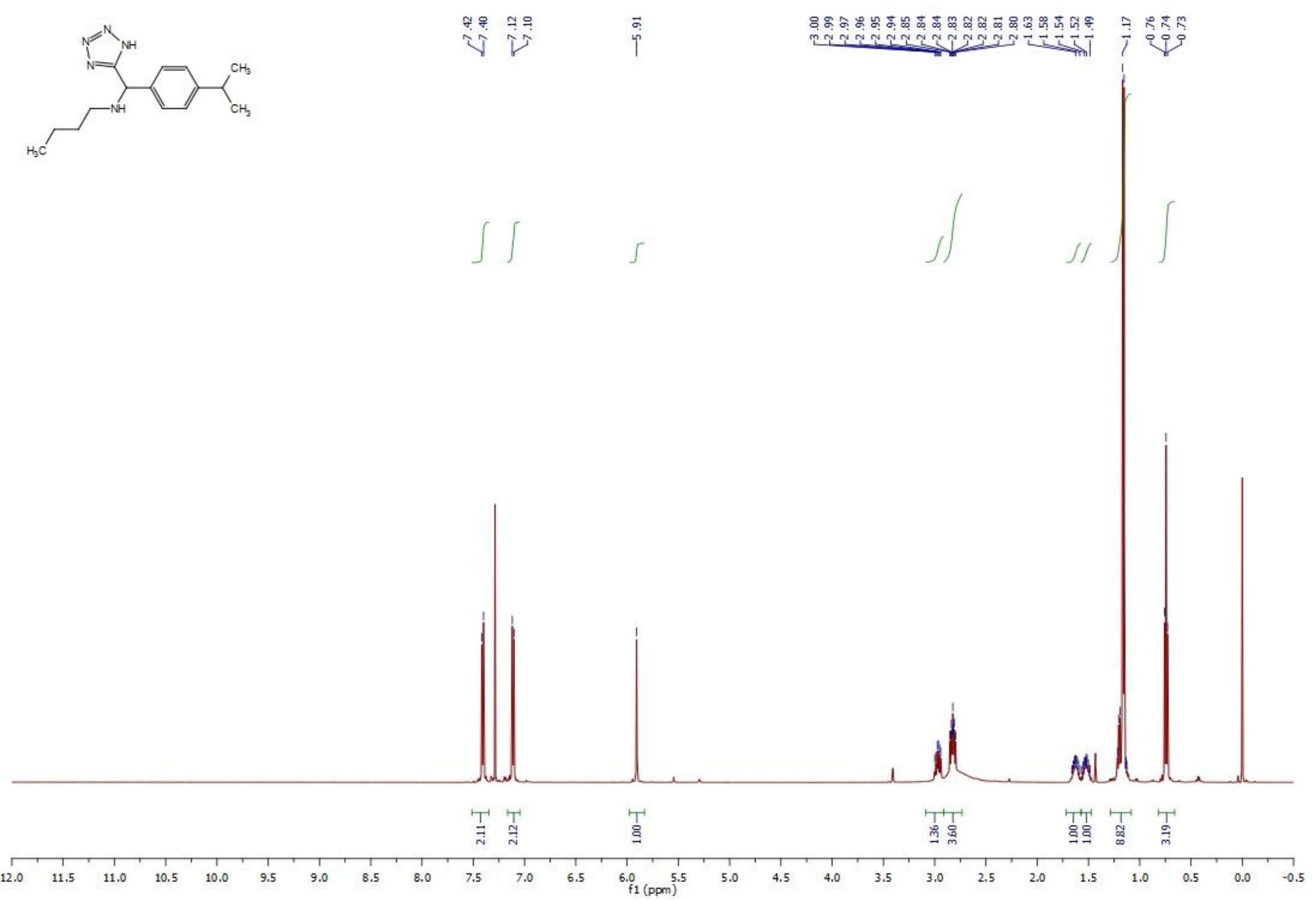



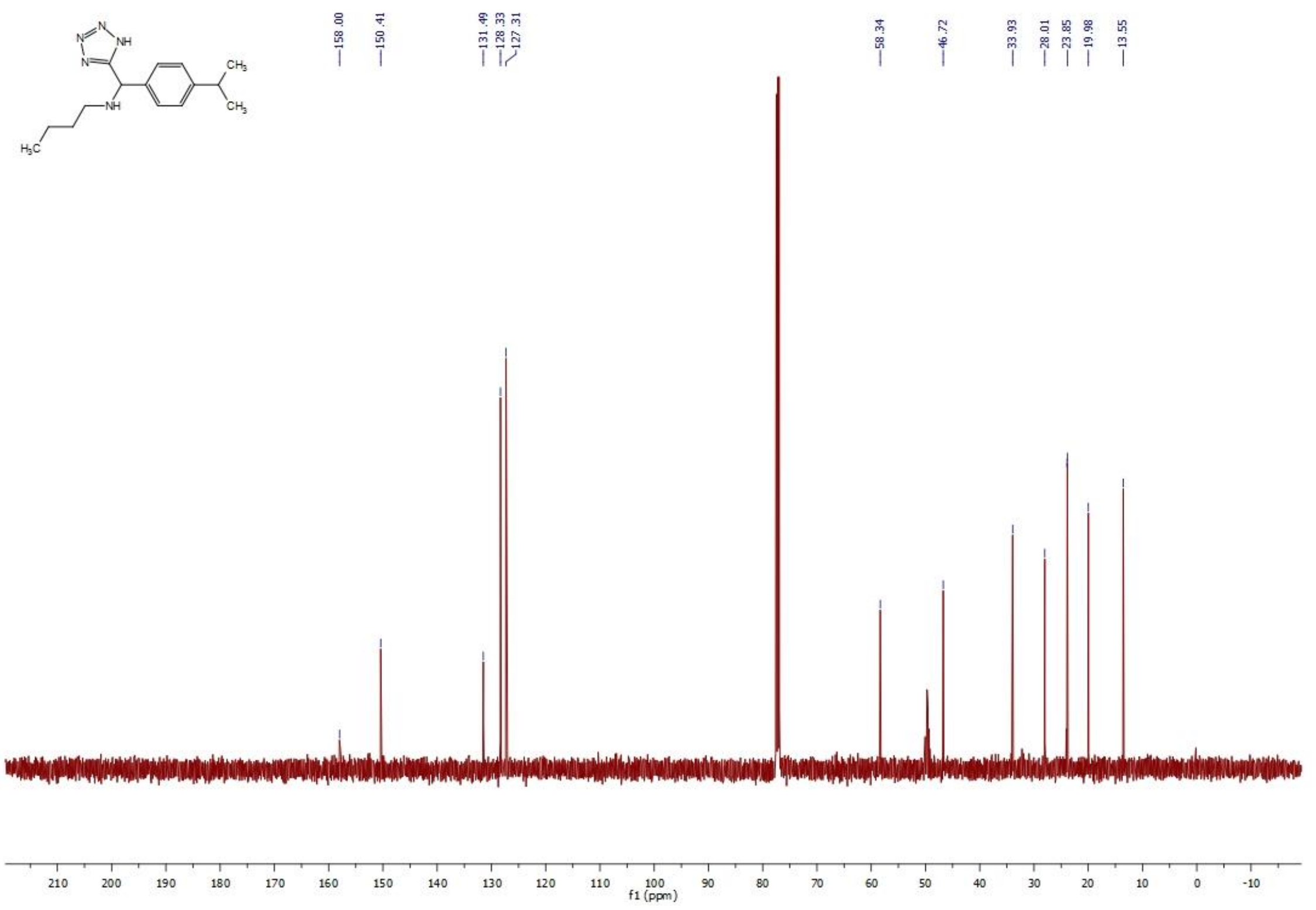
N-(cyclohexyl(1H-tetrazol-5-yl)methyl)-2,4,6-trimethylaniline (6b)
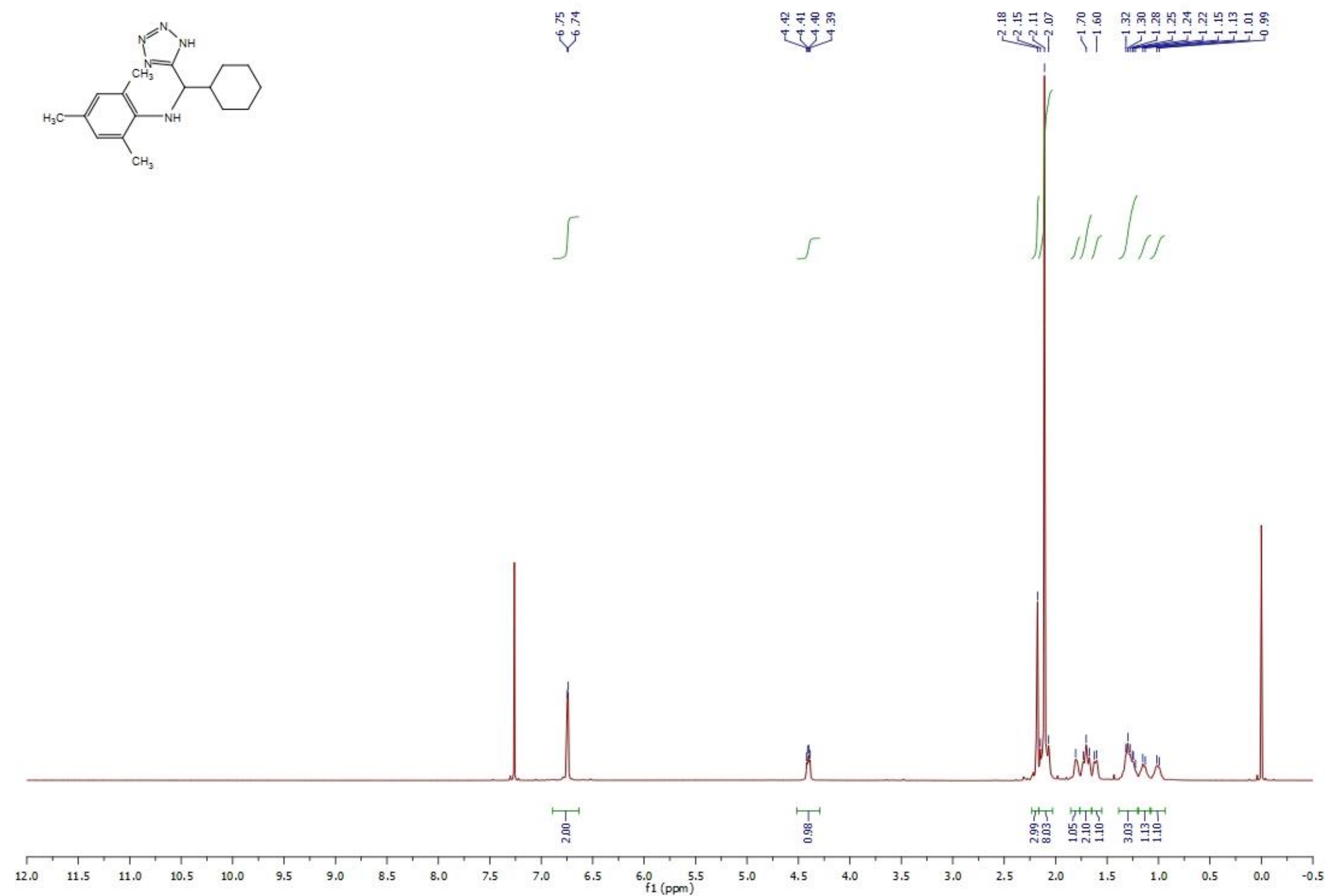


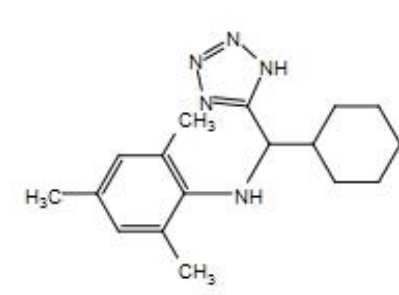

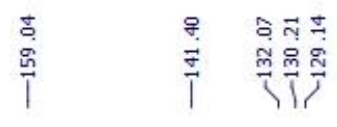

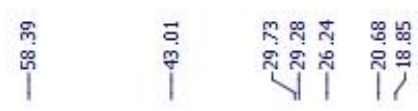
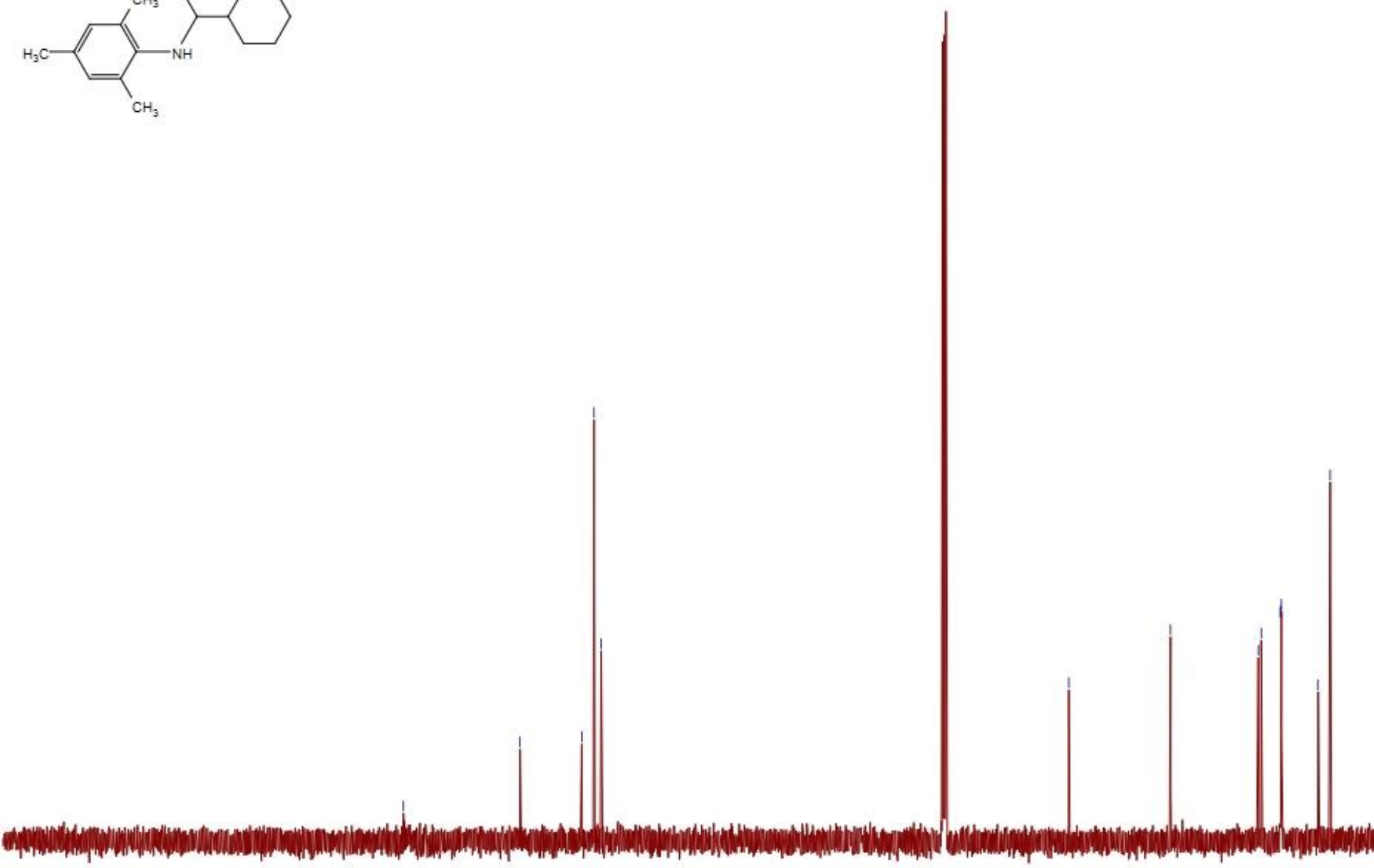
N-(1-(1H-tetrazol-5-yl)cyclohexyl)aniline (6c)

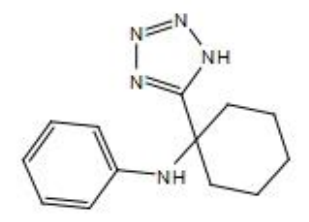

可

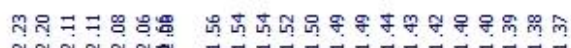
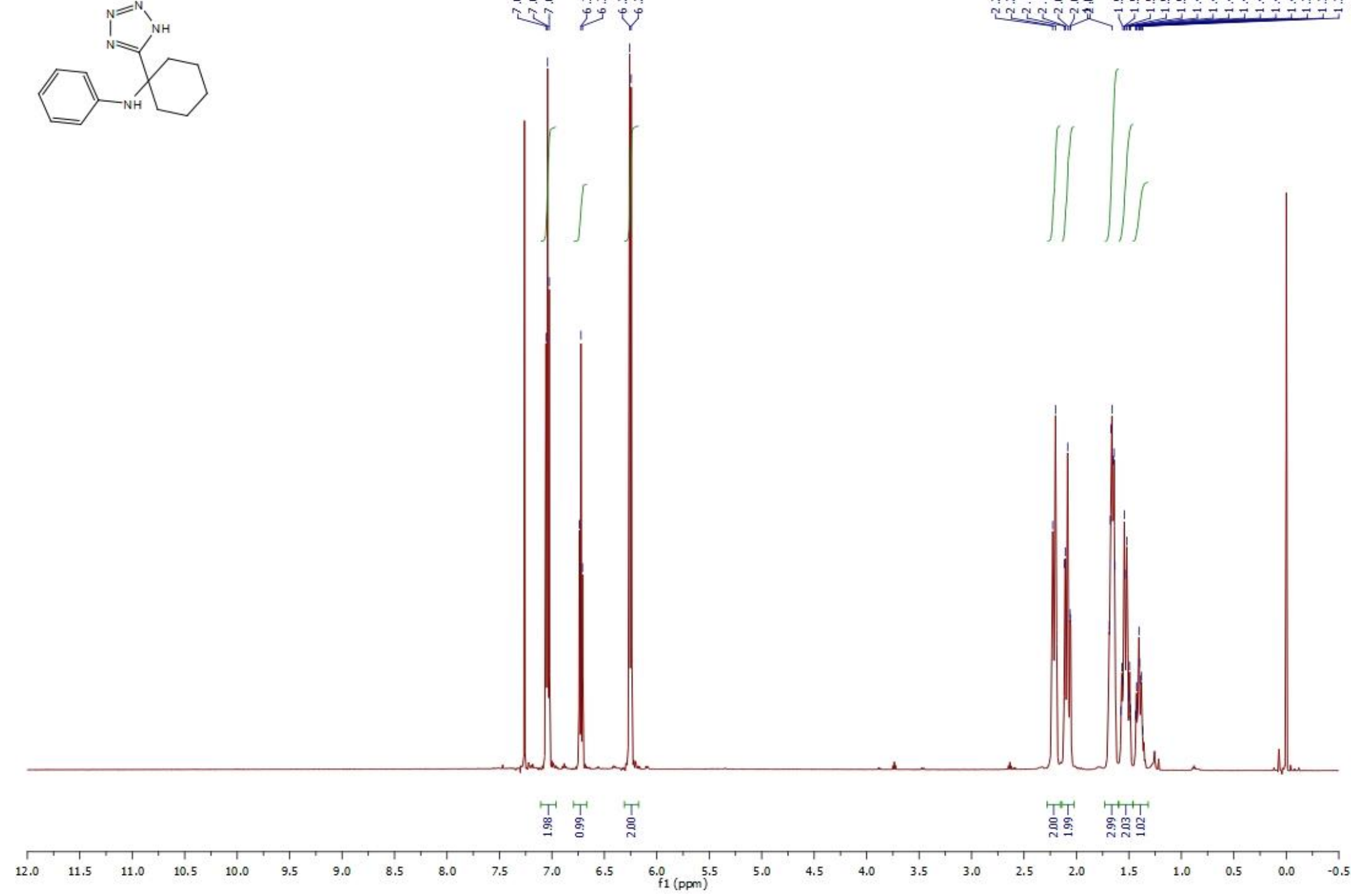

S44 


$$
\text { all }
$$




$$
\text { Ti, }
$$




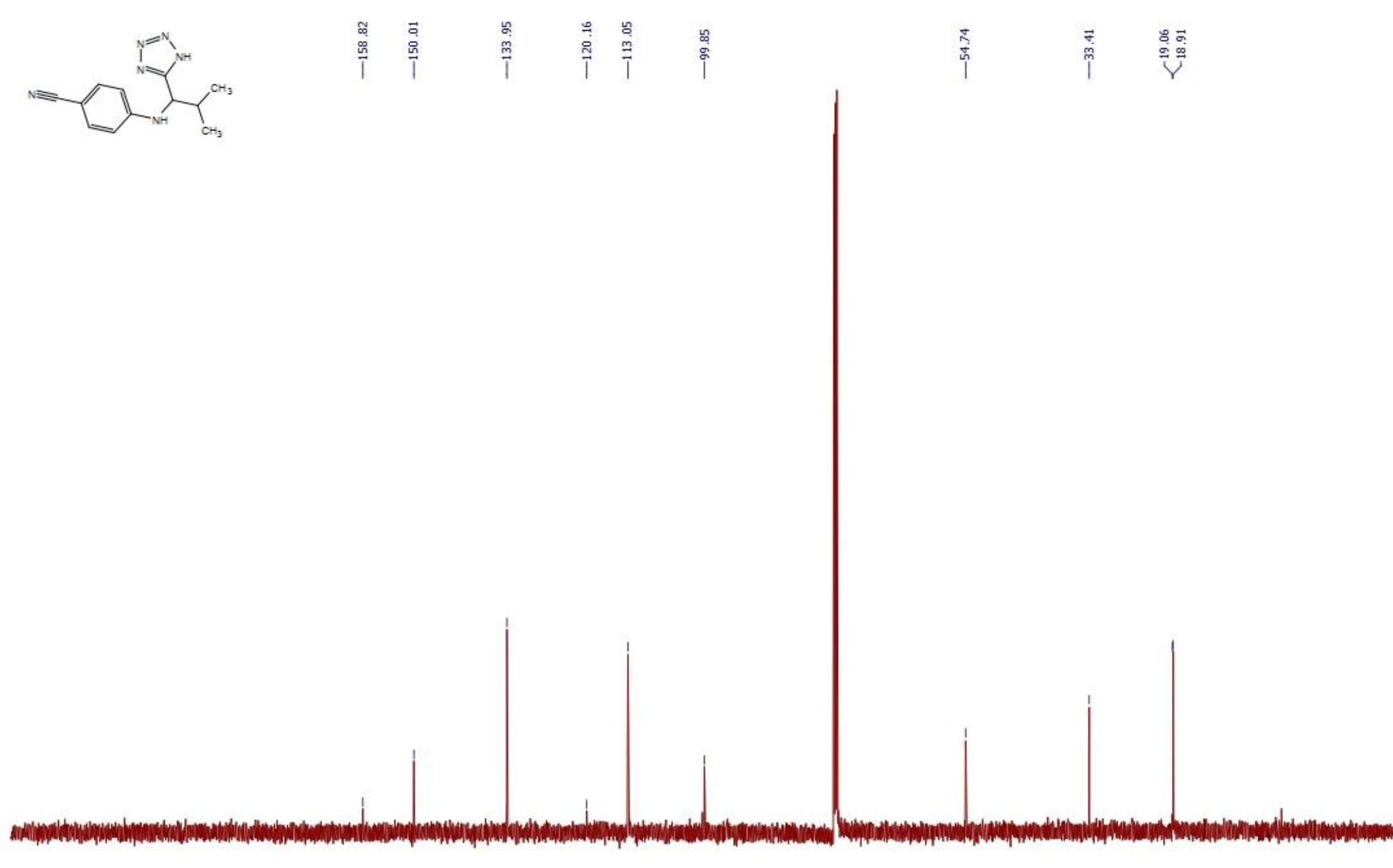


5-(benzo[d][1,3]dioxol-5-yl(pyrrolidin-1-yl)methyl)-1H-tetrazole (6e)

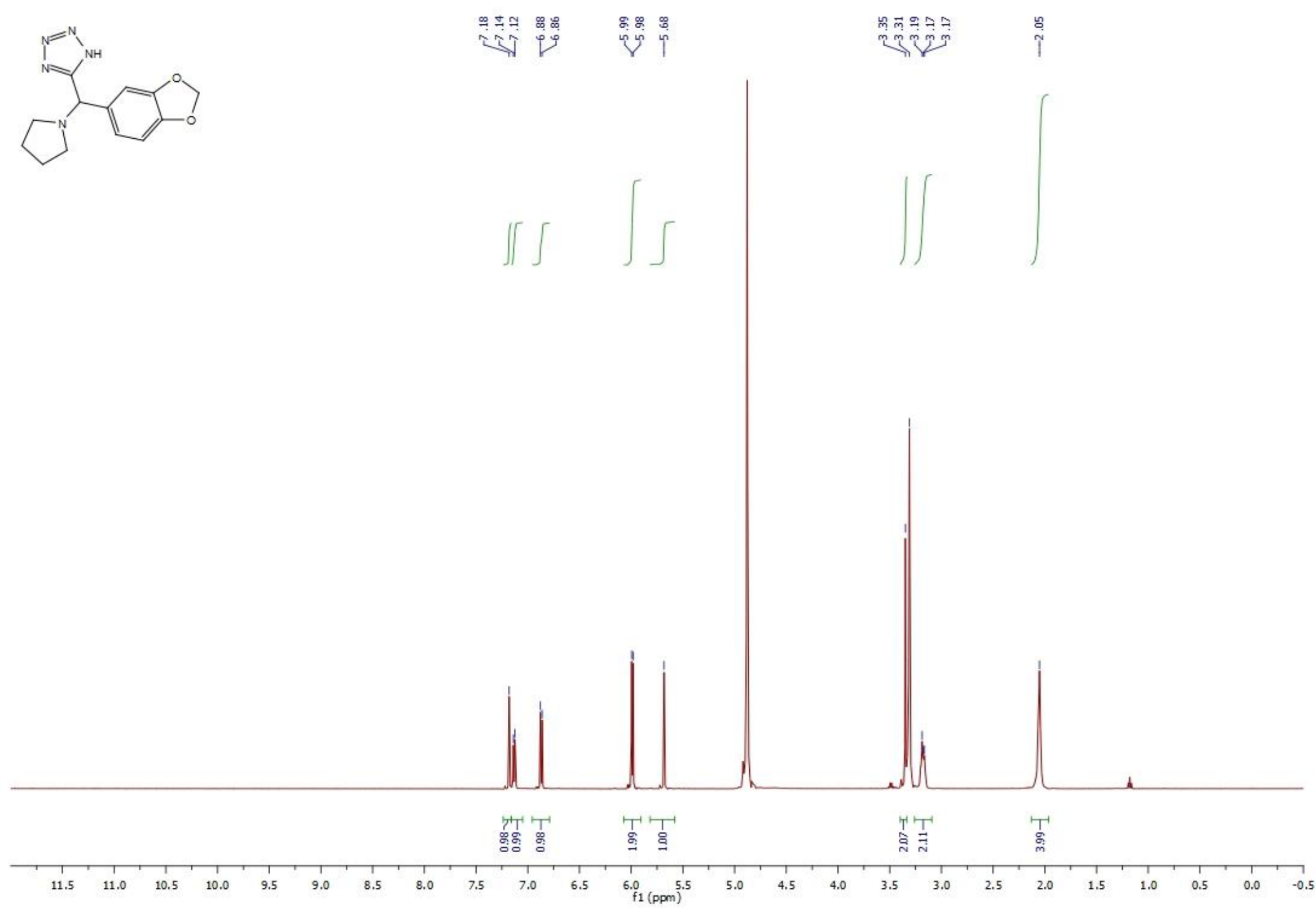



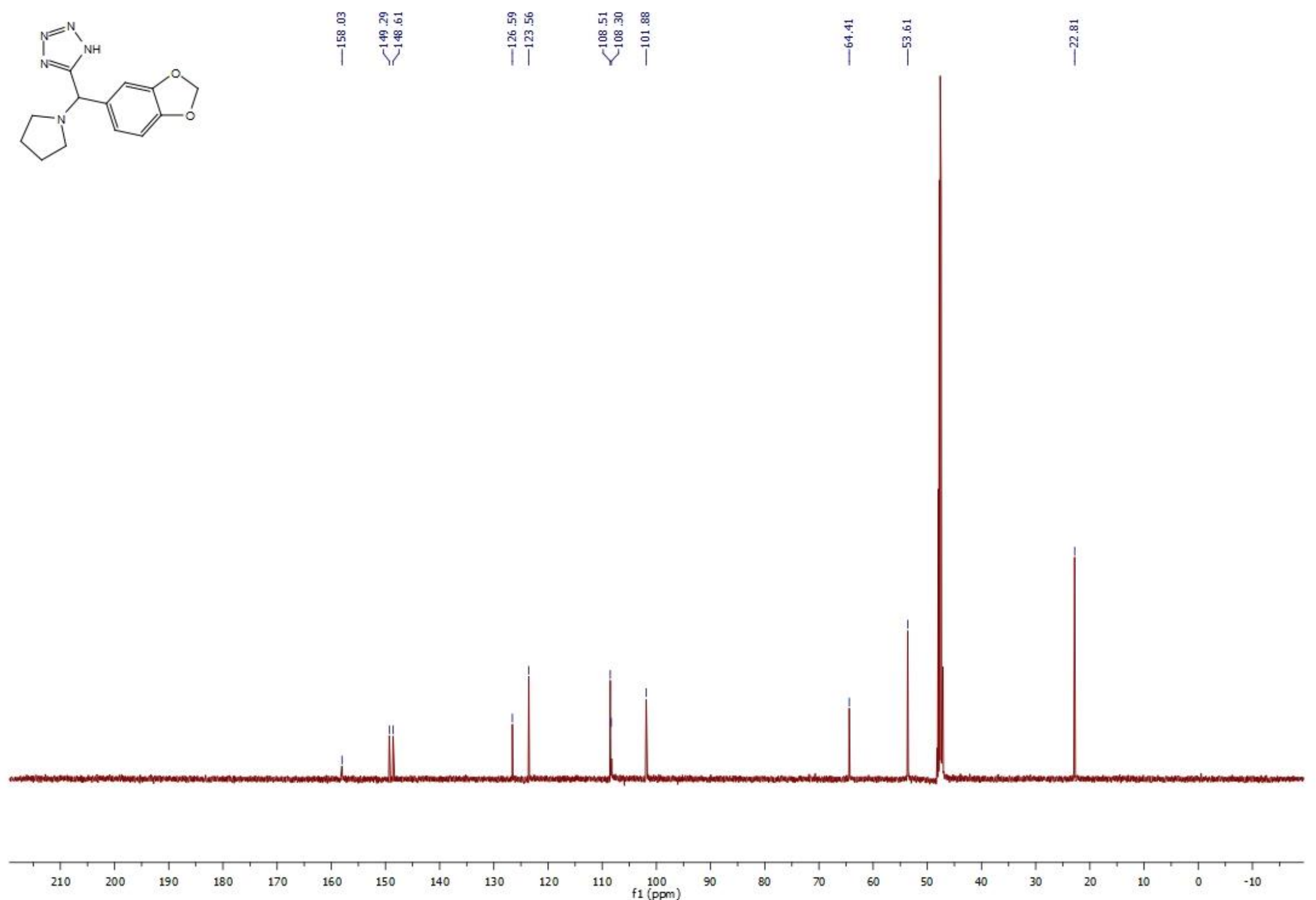
4-(naphthalen-1-yl(1H-tetrazol-5-yl)methyl)morpholine (6f)

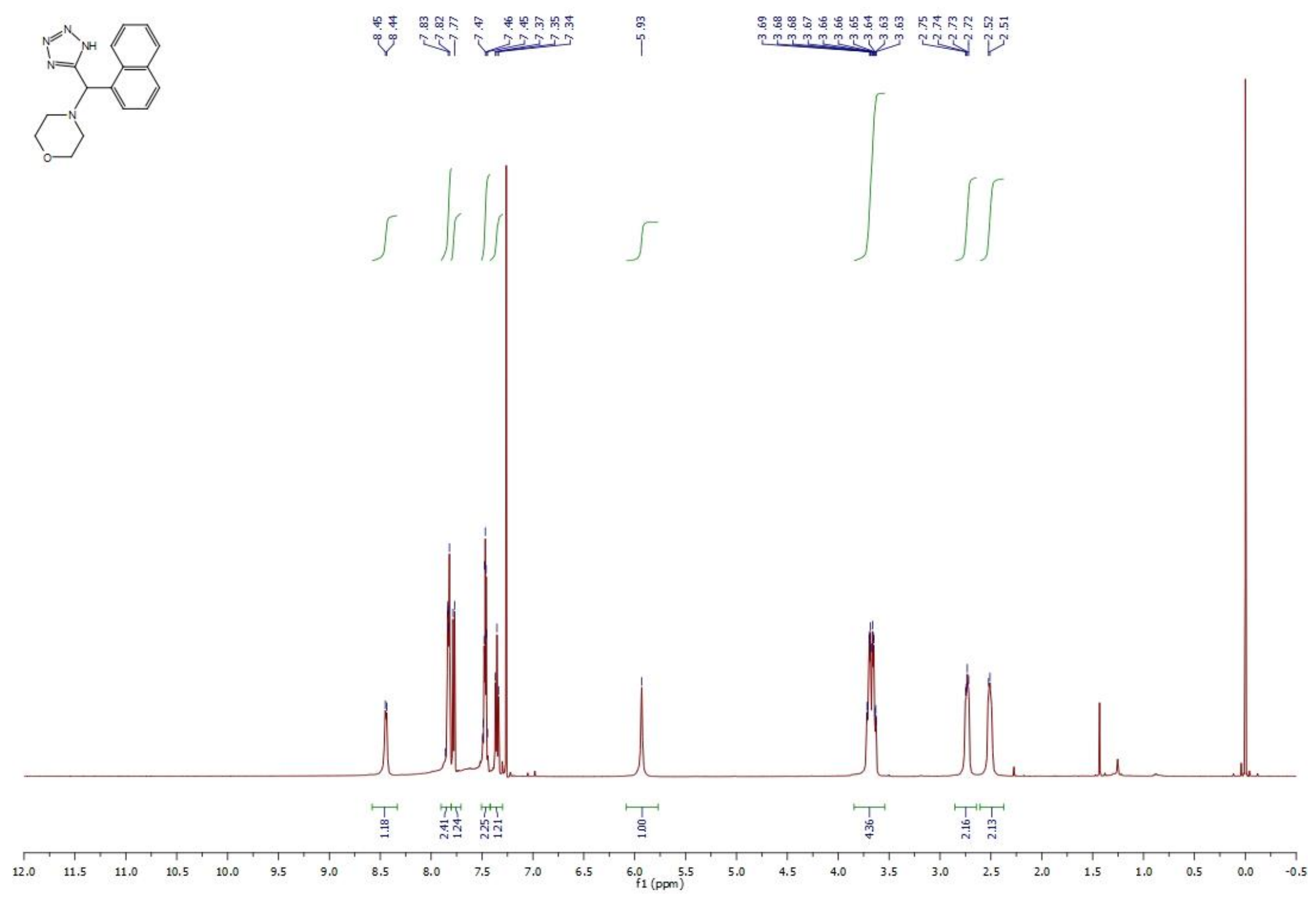




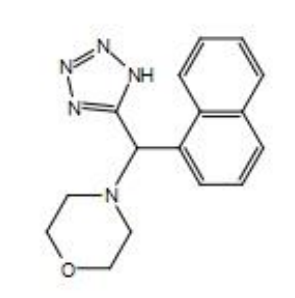

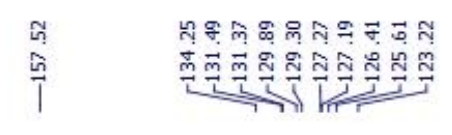

1
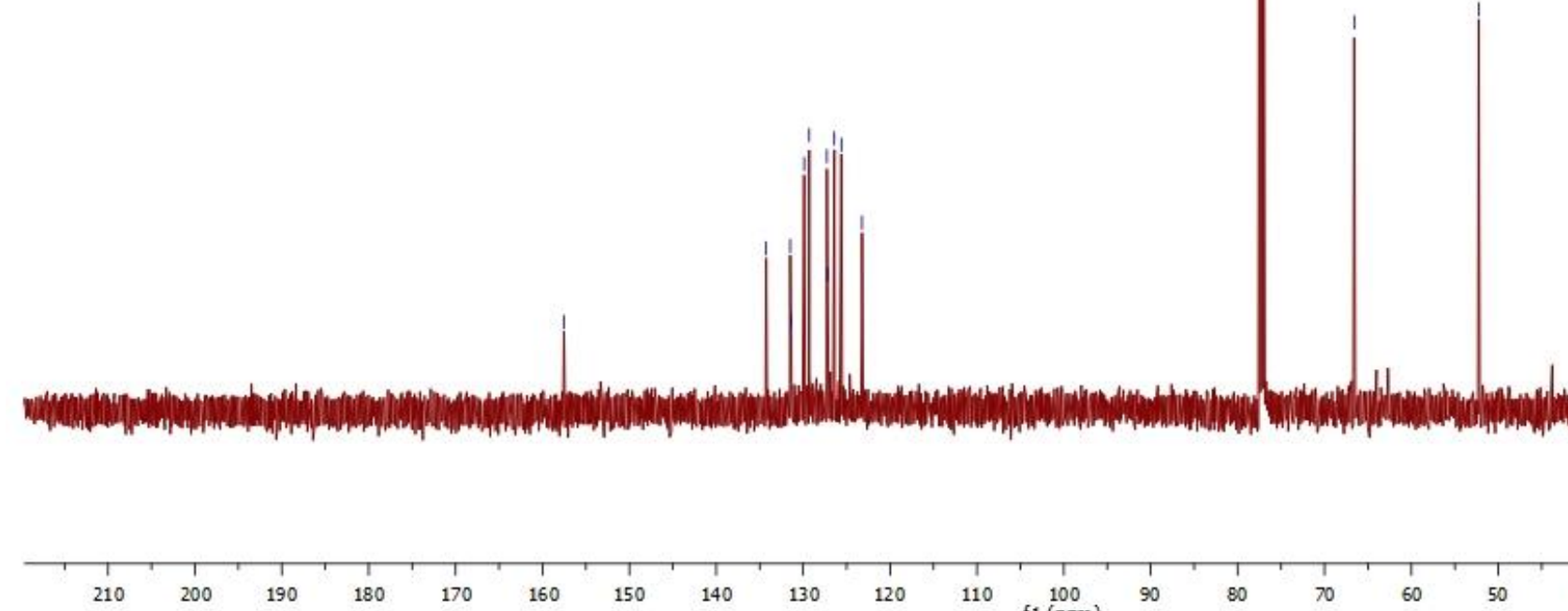

$160 \quad 150$

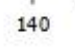

$130 \quad 120$

110
$\mathrm{f} 1(\mathrm{ppm})$
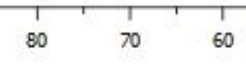

$60 \quad 50$ 
tert-butyl 4-(2-phenyl-1-(1H-tetrazol-5-yl)ethyl)piperazine-1-carboxylate (6g)

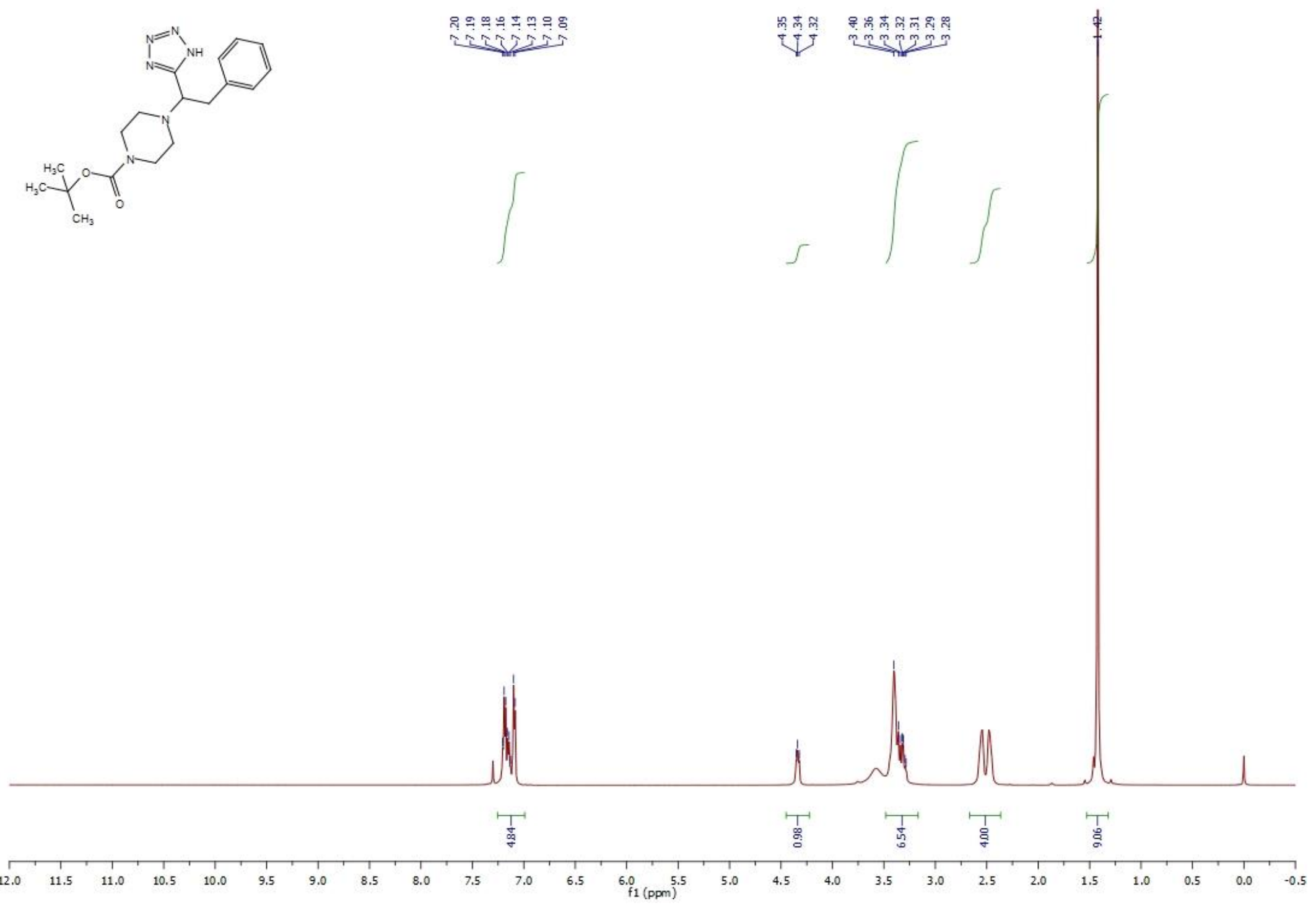



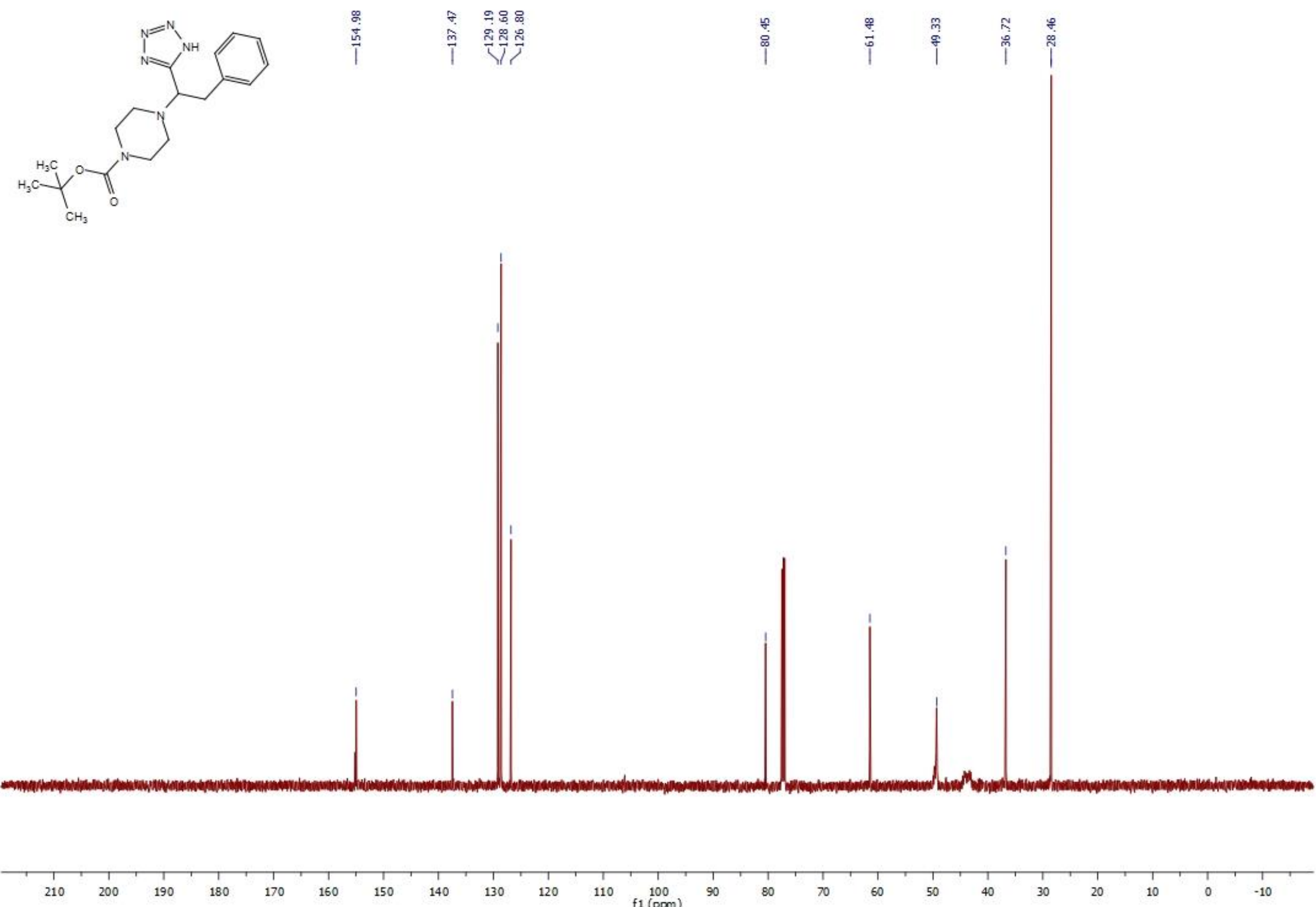
$N-((1 H$-tetrazol-5-yl)methyl)-1,1,1-triphenylmethanamine (6h)

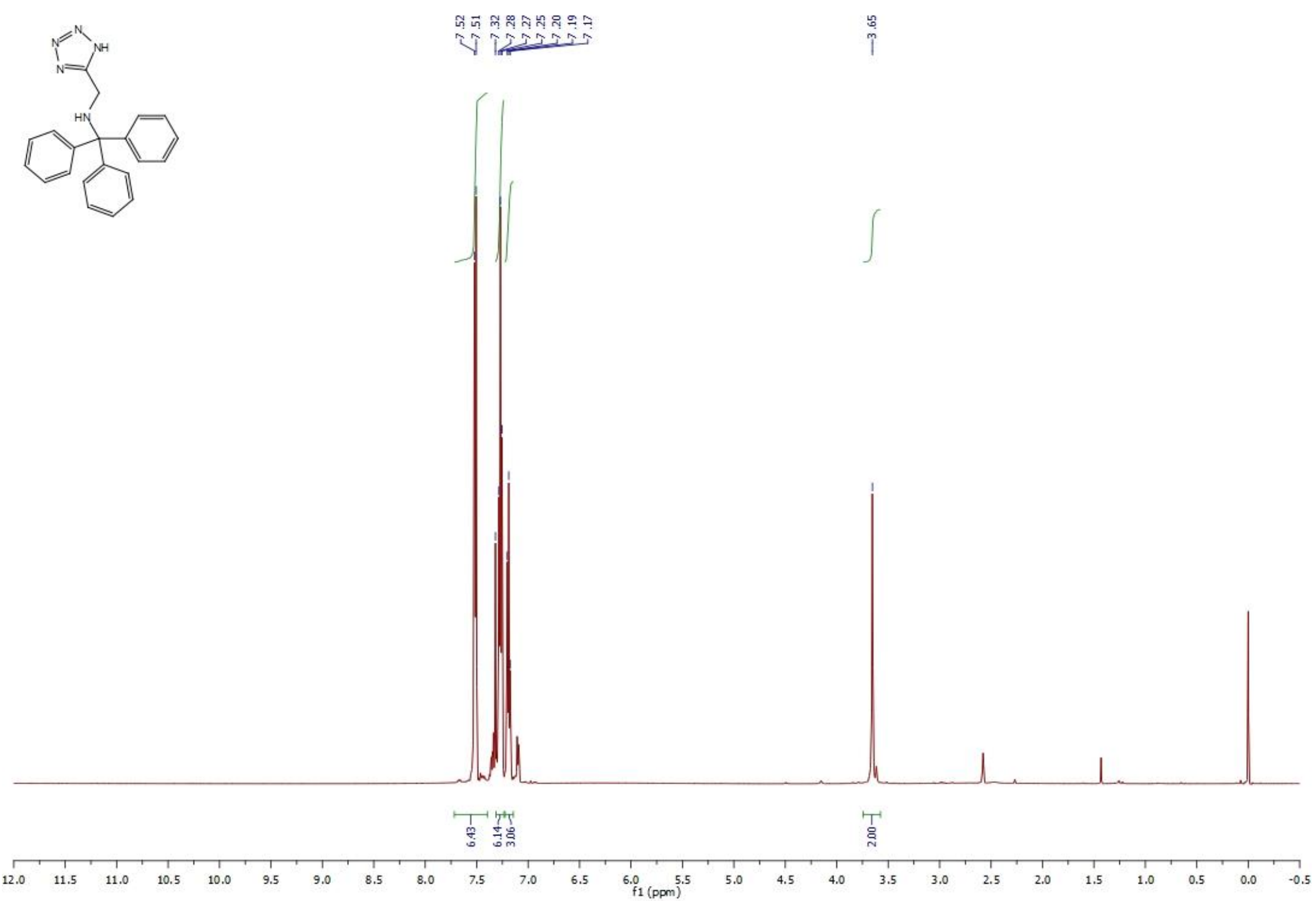



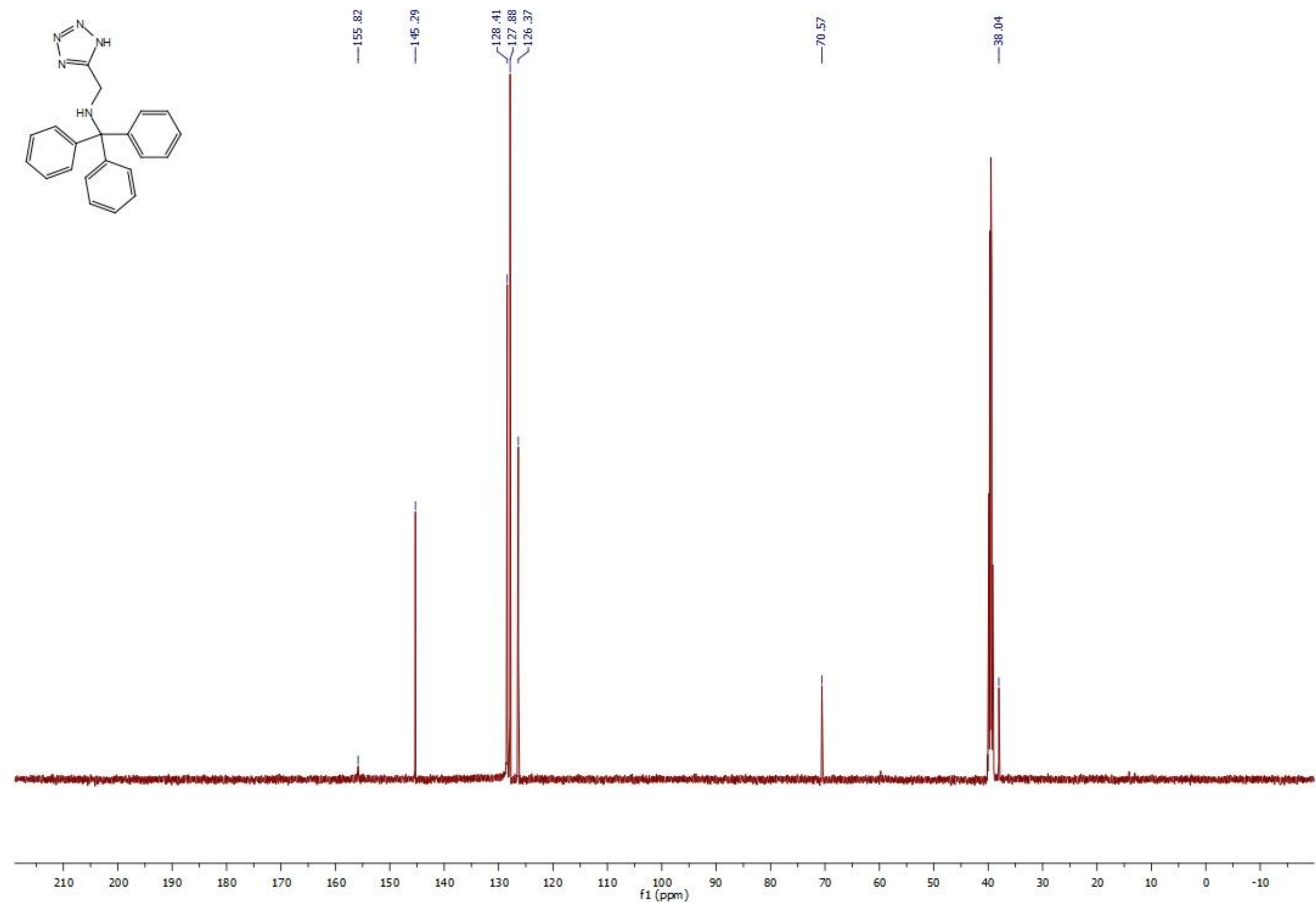
2-(1H-tetrazol-5-yl)-N-(thiophen-2-ylmethyl)propan-2-amine (6i)

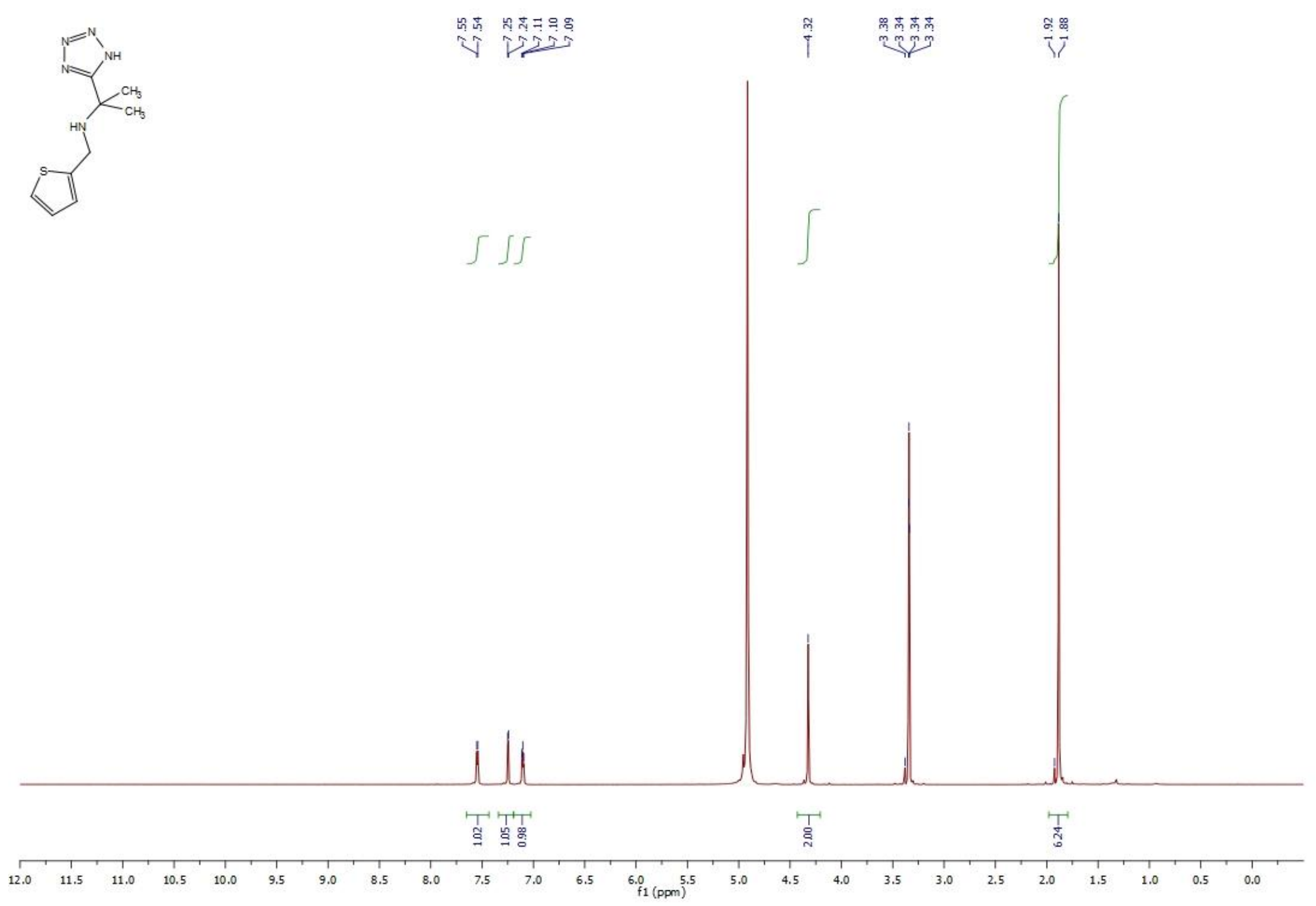




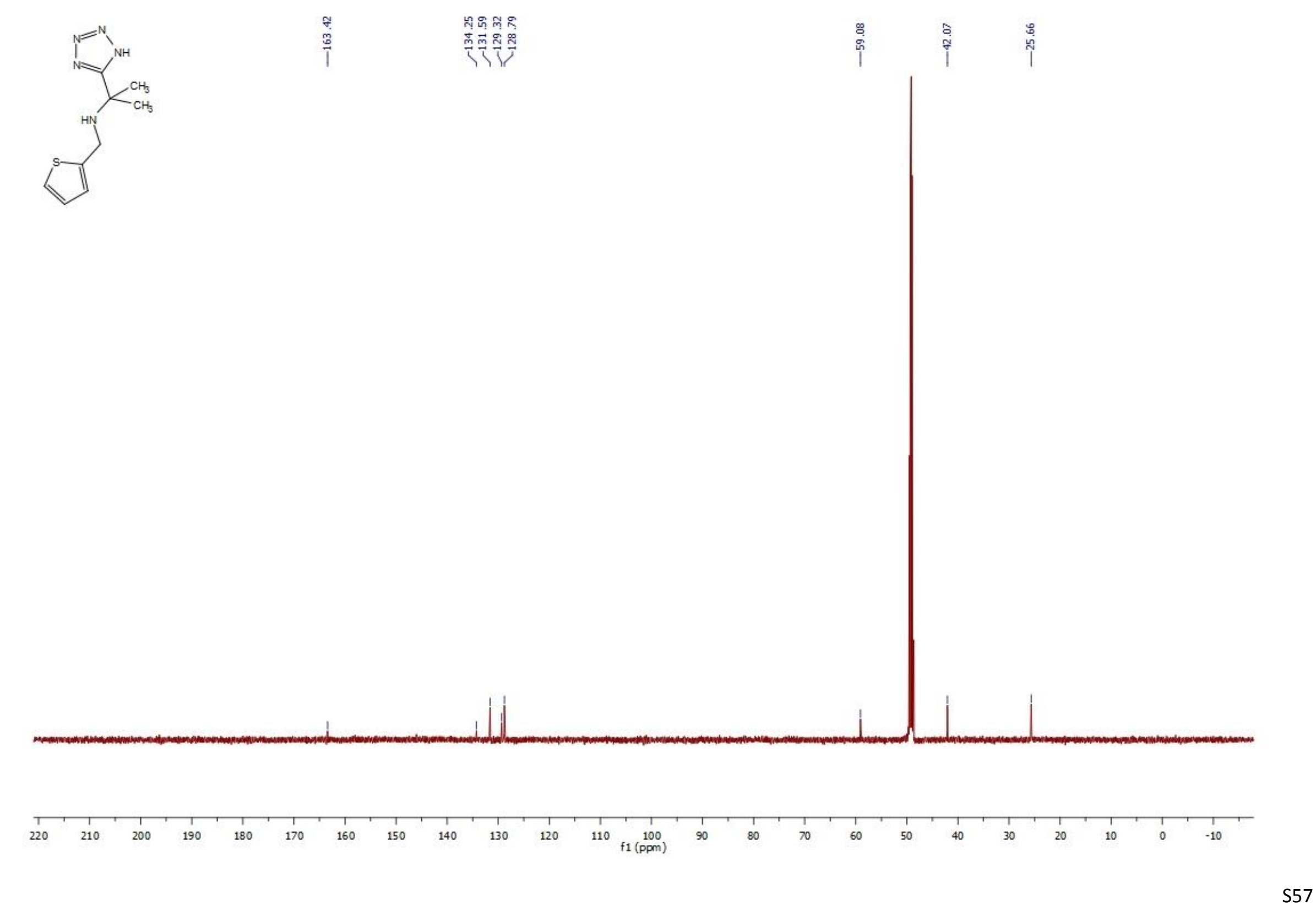


$N$-(benzo[b]thiophen-2-yl(1H-tetrazol-5-yl)methyl)-2-methylpropan-2-amine (6j)

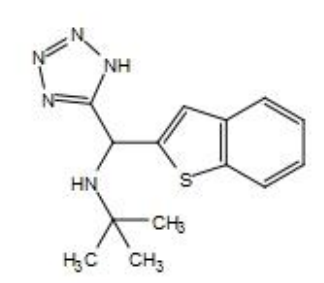

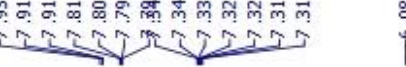
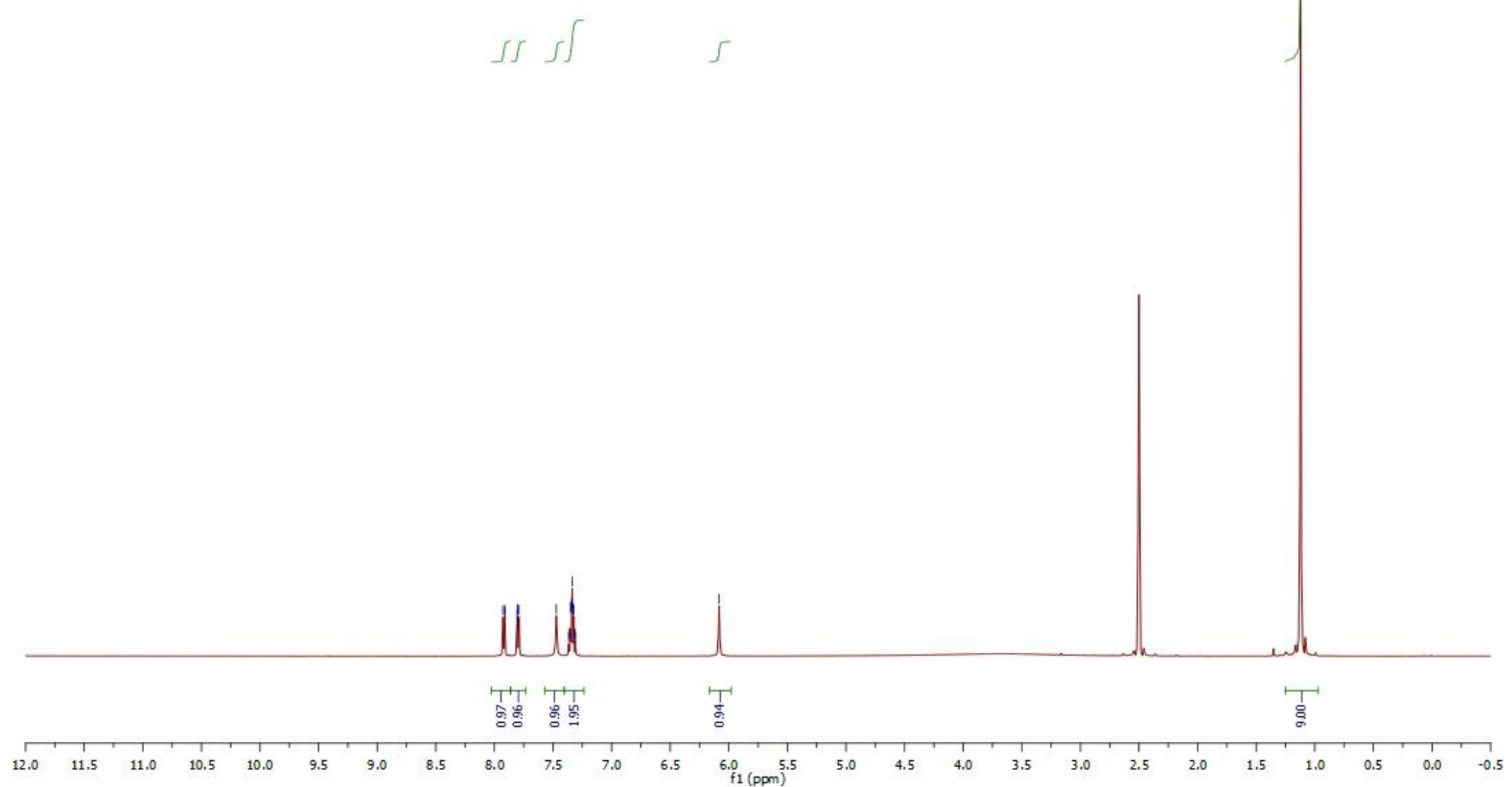


$$
\cdots
$$


2-((pyridin-3-yl(1H-tetrazol-5-yl)methyl)amino)ethan-1-ol (6k)
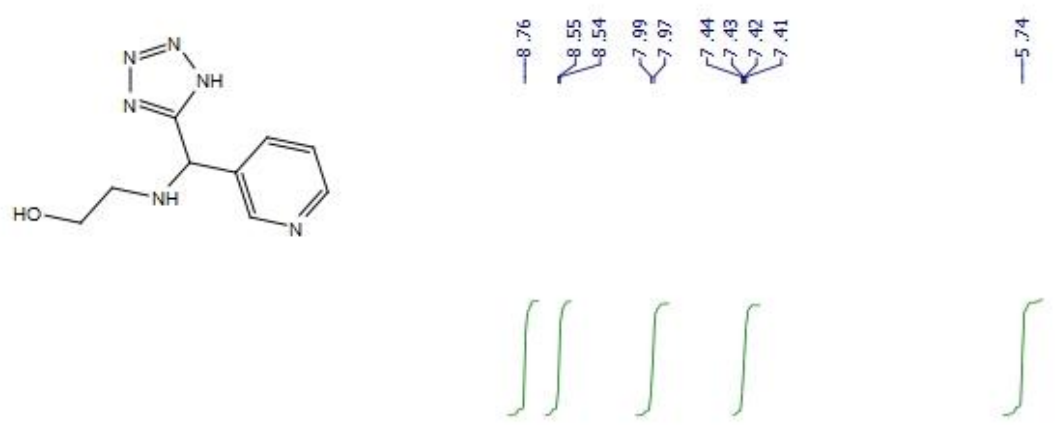

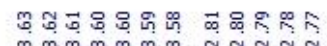

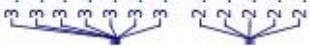

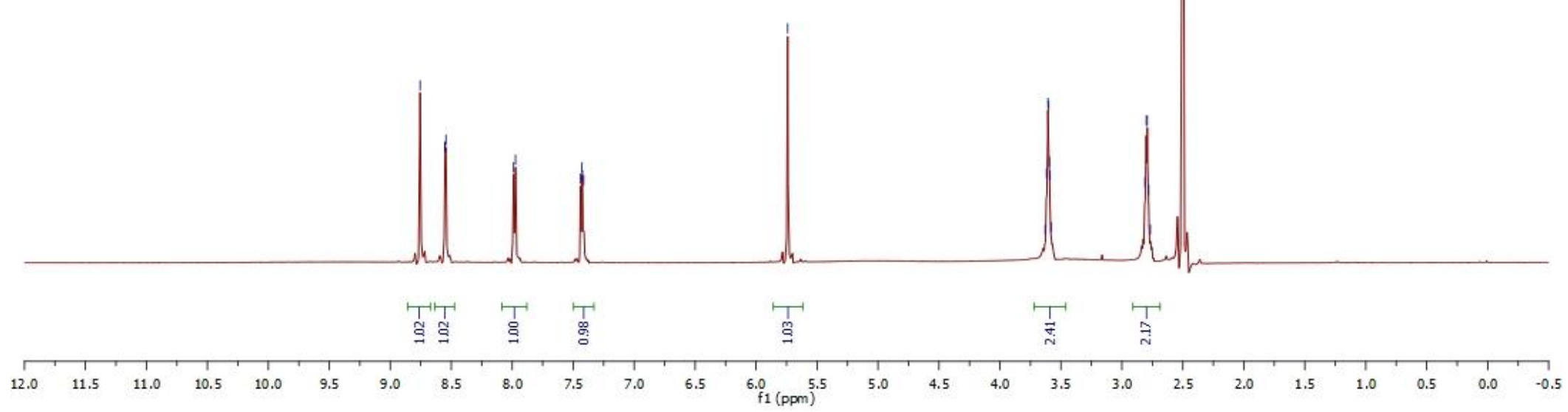

S60 


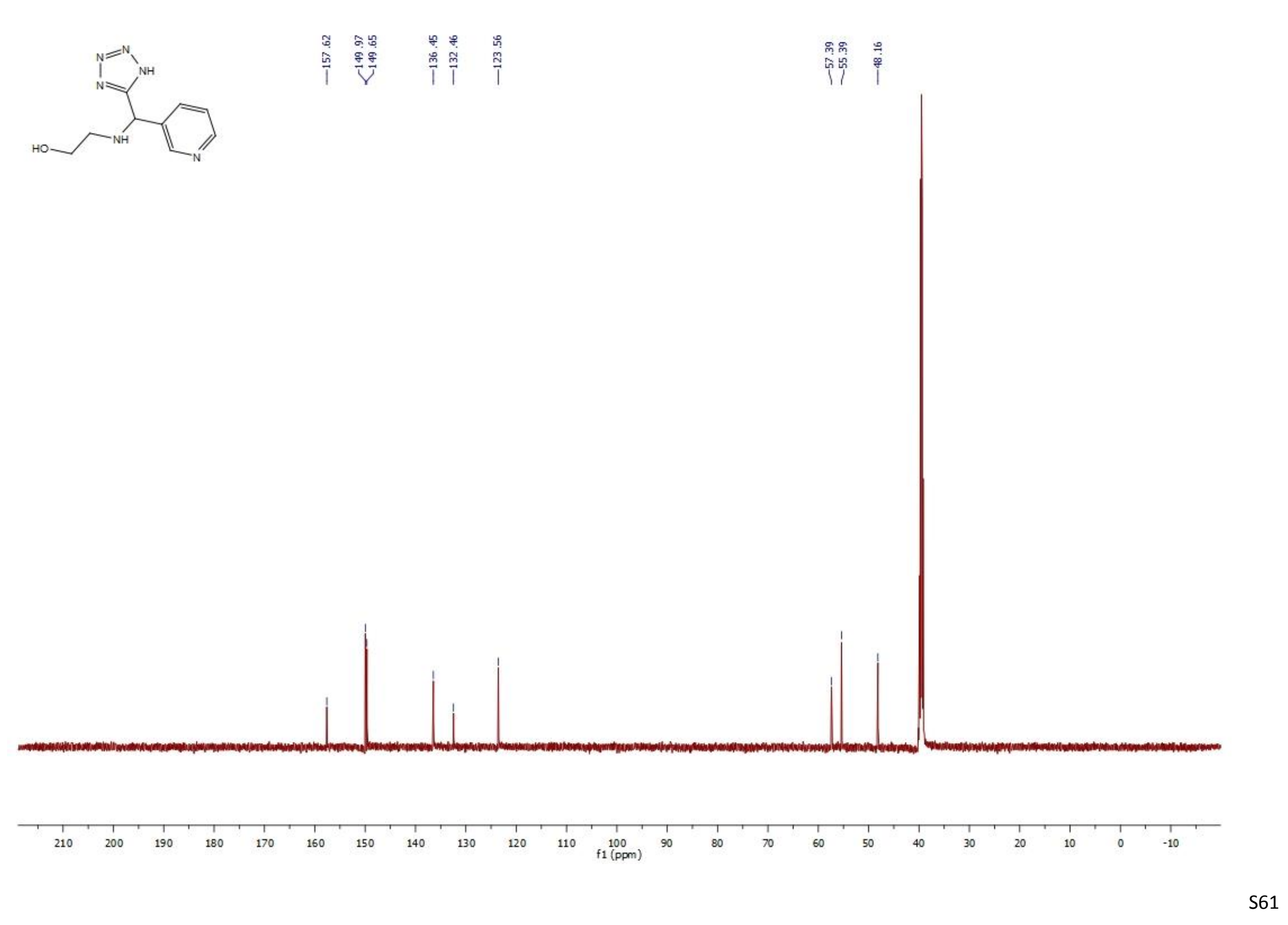


N-benzyl-N-(1-((2-cyanoethyl)amino)-2-methylpropyl)benzamide (7)

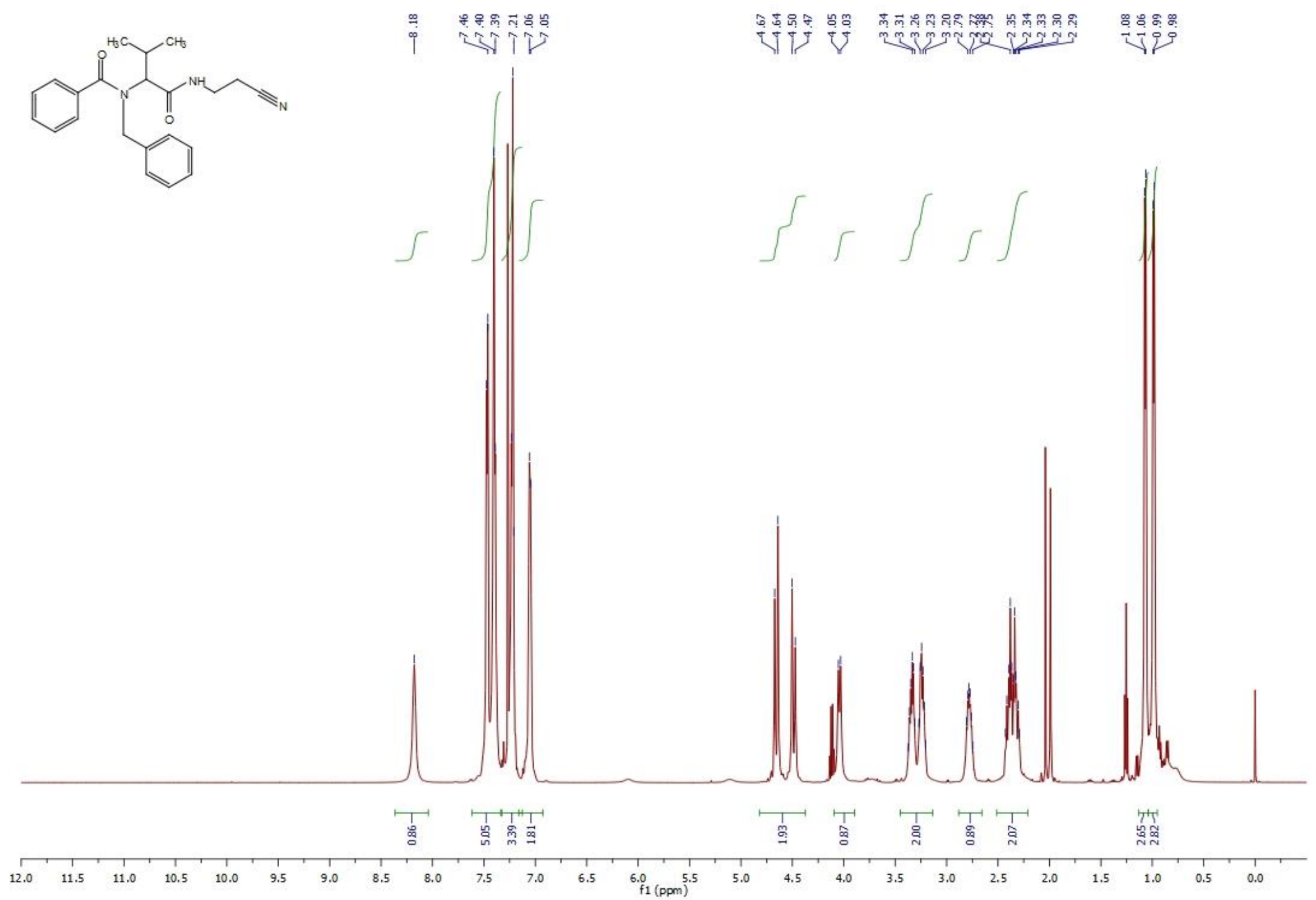




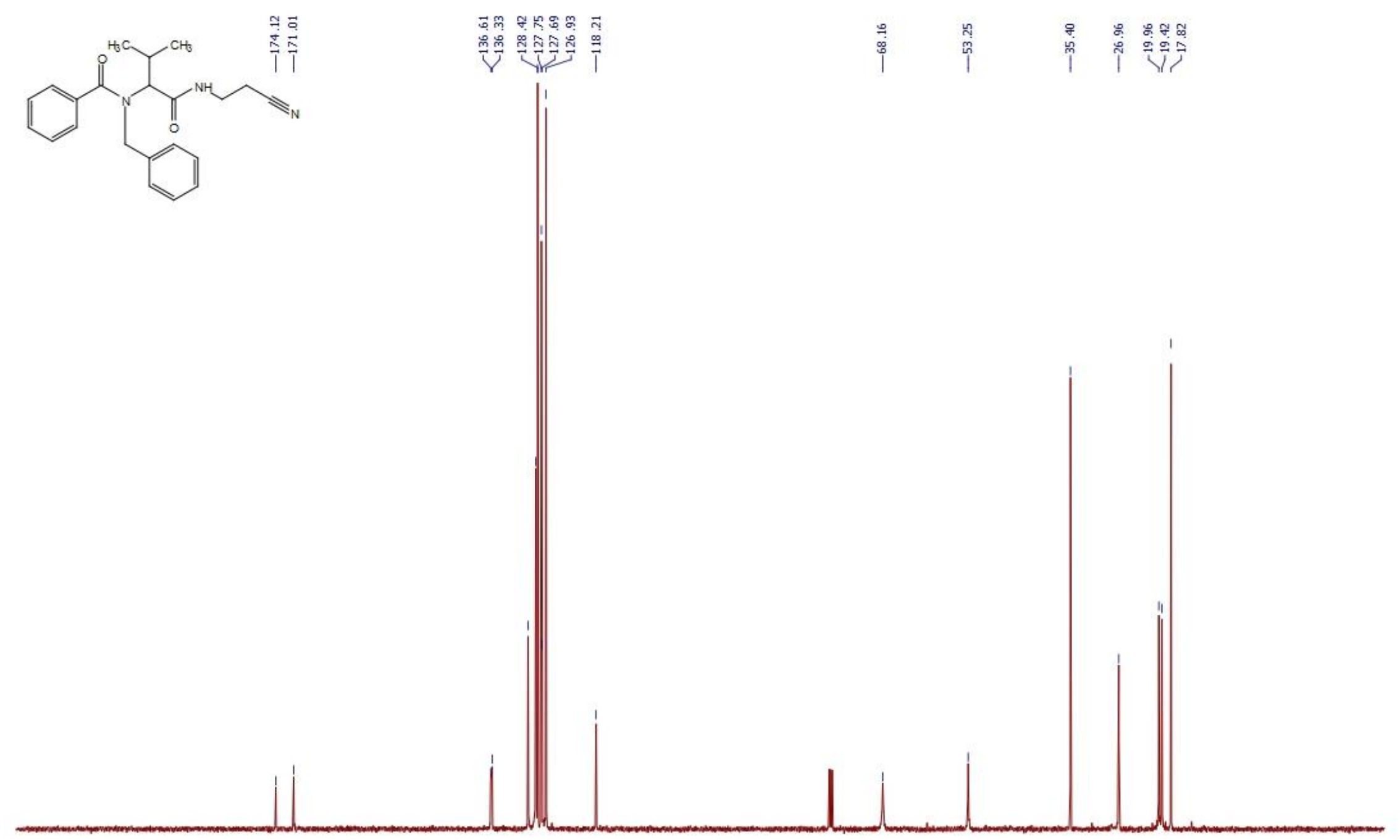


1-(2-phenyl-1-(1H-tetrazol-5-yl)ethyl)piperazine hydrochloride (8)

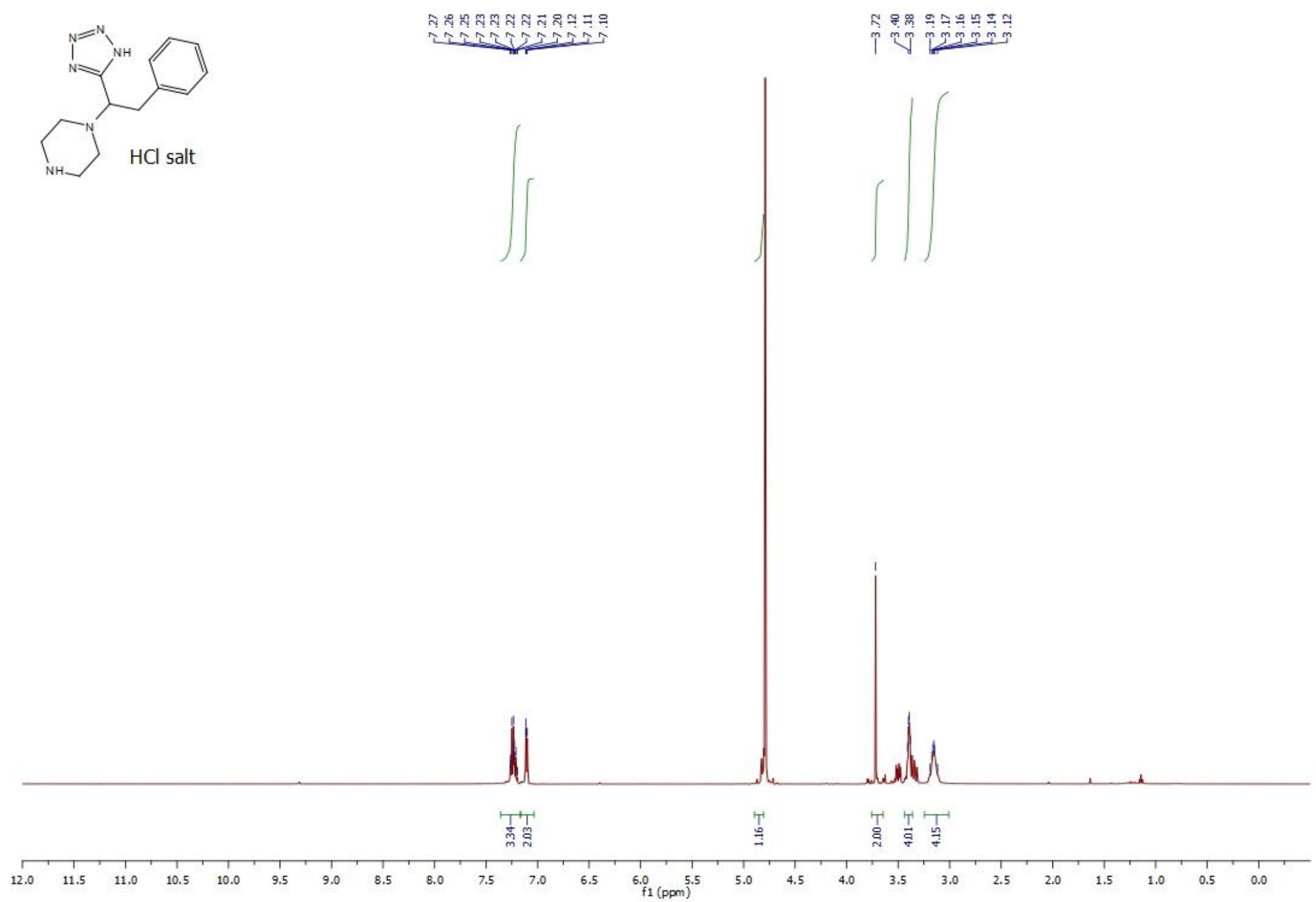



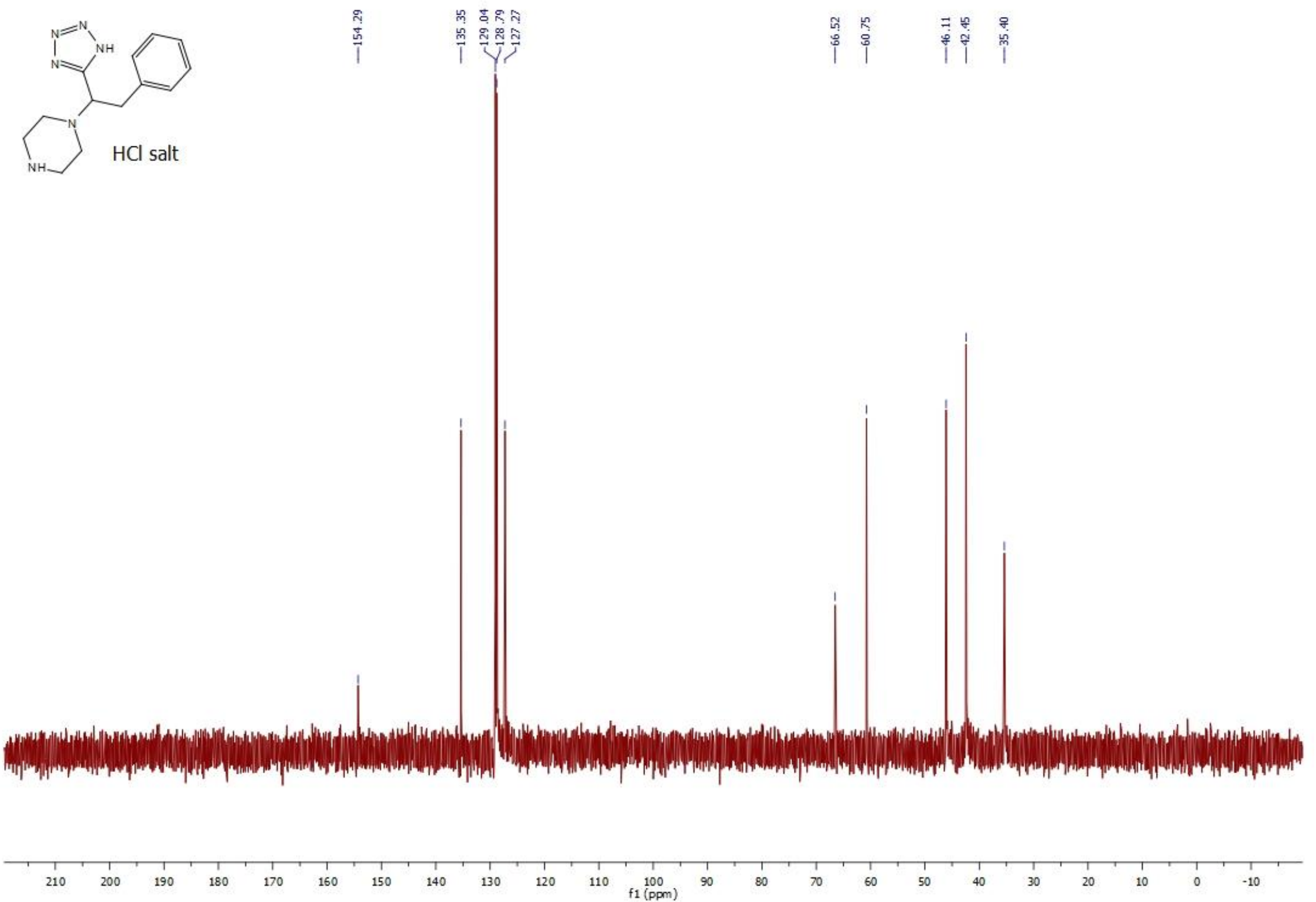
(1H-tetrazol-5-yl)methanamine hydrochloride (9)

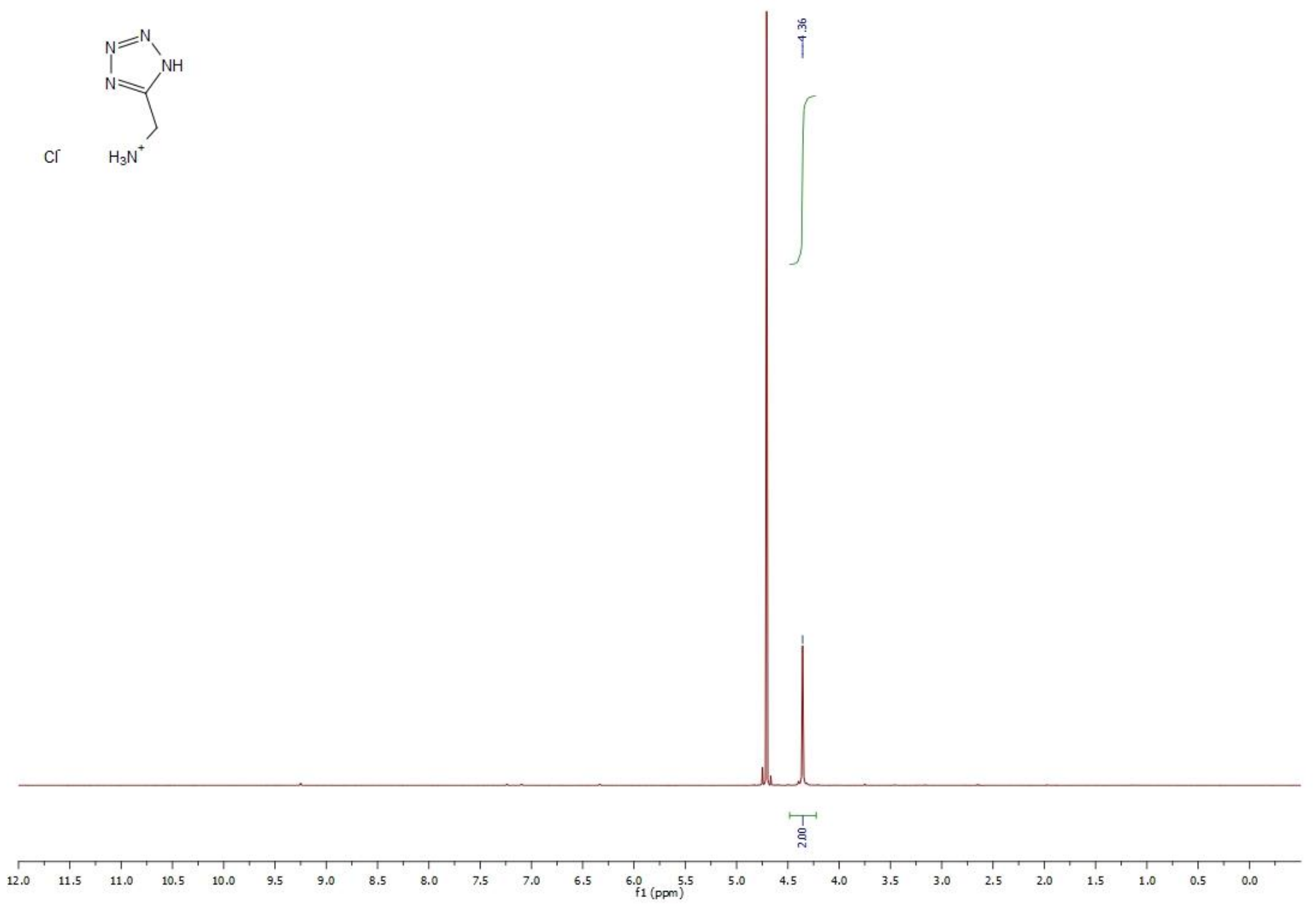




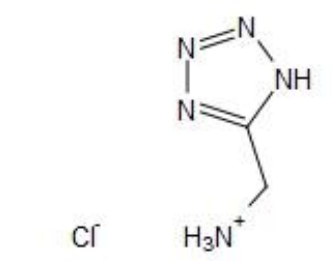

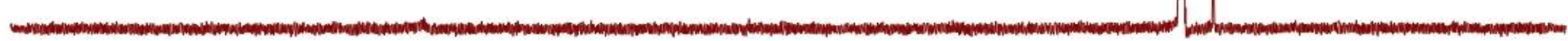

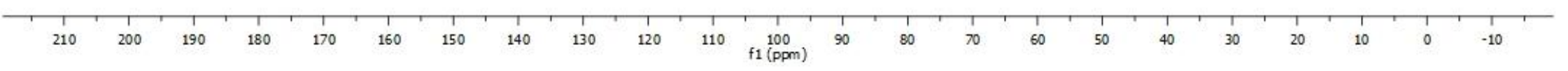




\section{Single Crystal X-Ray Structure Determination of Compound 5c}

X-ray diffraction data for single crystal of compound $\mathbf{5 c}$ was collected using SuperNova (Rigaku - Oxford Diffraction) four circle diffractometer with a mirror monochromator and a microfocus MoKa radiation source $(\lambda=1.7107 \AA)$. Additionally, the diffractometer was equipped with a CryoJet HT cryostat system (Oxford Instruments) allowing low temperature experiments. Single crystal X-ray experiments was performed at room temperature. The obtained data set was processed with CrysAlis ${ }^{\text {Pro }}$ software. ${ }^{2}$ The phase problem was solved by direct methods using SIR2004. ${ }^{3}$ Parameters of obtained model was refined by full-matrix least-squares on $\mathrm{F}^{2}$ using SHELXL-2014/6. ${ }^{4}$ Calculations were performed using WinGX integrated system (ver. 2014.1). ${ }^{5}$ Figures were prepared with Mercury 3.5 software. ${ }^{6}$

All non-hydrogen atoms were refined anisotropically. All hydrogen atoms attached to carbon atoms were positioned with the idealised geometry and refined using the riding model with the isotropic displacement parameter $\mathrm{U}_{\text {iso }}[\mathrm{H}]=1.2$ (or 1.5 (methyl groups only)) $U_{\text {eq }}[C]$. The position of hydrogen linked to the $N$ atoms was found on the difference Fourier map and refined with no restrains on the isotropic displacement parameter. Crystal data and structure refinement results for presented crystal structure is shown in Table S1 and the asymmetric unit is shown in Figure S1.

Crystallographic data for structure presented in this paper has been deposited with the Cambridge Crystallographic Data Centre as supplementary publication nos. CCDC 1479665 (5c). Copies of the data can be obtained, free of charge, on application to CCDC, 12 Union Road, Cambridge CB2 1EZ, UK, (fax: +44-(0)1223-336033 or e-mail: deposit@ccdc.cam.ac.uk). 
Table S1. Crystal data and structure refinement results for compounds 5c.

\begin{tabular}{|c|c|}
\hline & 5c \\
\hline Empirical moiety formula & $\mathrm{C}_{16} \mathrm{H}_{20} \mathrm{~N}_{6}$ \\
\hline Formula weight $[\mathrm{g} / \mathrm{mol}]$ & 296.38 \\
\hline Crystal system & Monoclinic \\
\hline Space group & $\mathrm{P} 2_{1} / a$ \\
\hline Unite cell dimensions & $\begin{array}{c}\mathrm{a}=12.3916(7) \AA \\
\mathrm{b}=9.7149(5) \AA \\
\mathrm{c}=13.2453(10) \AA \\
\mathrm{a}=90^{\circ} \\
\beta=94.592(6)^{\circ} \\
\gamma=90^{\circ}\end{array}$ \\
\hline Volume $\left[\AA^{3}\right]$ & $1589.39(17)$ \\
\hline Z & 4 \\
\hline$D_{\text {calc }}\left[\mathrm{Mg} / \mathrm{m}^{3}\right]$ & 1.239 \\
\hline$\mu\left[\mathrm{mm}^{-1}\right]$ & 0.079 \\
\hline$F(000)$ & 632 \\
\hline Crystal size $\left[\mathrm{mm}^{3}\right]$ & $0.5 \times 0.2 \times 0.1$ \\
\hline$\Theta$ range & $3.00^{\circ}$ to $28.50^{\circ}$ \\
\hline Index ranges & $\begin{array}{l}-16 \leq \mathrm{h} \leq 15 \\
-11 \leq \mathrm{k} \leq 12 \\
-16 \leq \mathrm{I} \leq 17\end{array}$ \\
\hline Refl. collected & 8535 \\
\hline Independent reflections & $\begin{array}{c}1587 \\
{[R \text { (int) }=0.0106]}\end{array}$ \\
\hline Completeness [\%] to $\Theta$ & $99.8\left(\Theta 26.31^{\circ}\right)$ \\
\hline Absorption correction & Multi-scan \\
\hline Tmin. and Tmax. & 0.269 and 1.000 \\
\hline $\begin{array}{c}\text { Data/ } \\
\text { restraints/parameters }\end{array}$ & 4022 / 0 / 200 \\
\hline GooF on F2 & 0.979 \\
\hline $\begin{array}{l}\text { Final } R \text { indices } \\
{[I>2 \text { sigma }(\mathrm{I})]}\end{array}$ & $\begin{array}{l}\mathrm{R} 1=0.0821 \\
\mathrm{WR} 2=0.1821\end{array}$ \\
\hline $\mathrm{R}$ indices (all data) & $\begin{array}{l}\mathrm{R} 1=0.1803 \\
\mathrm{WR} 2=0.2405\end{array}$ \\
\hline$\Delta \rho_{\max }, \Delta \rho_{\min }\left[\mathrm{e} \cdot \AA^{-3}\right]$ & 0.051 and -0.261 \\
\hline
\end{tabular}




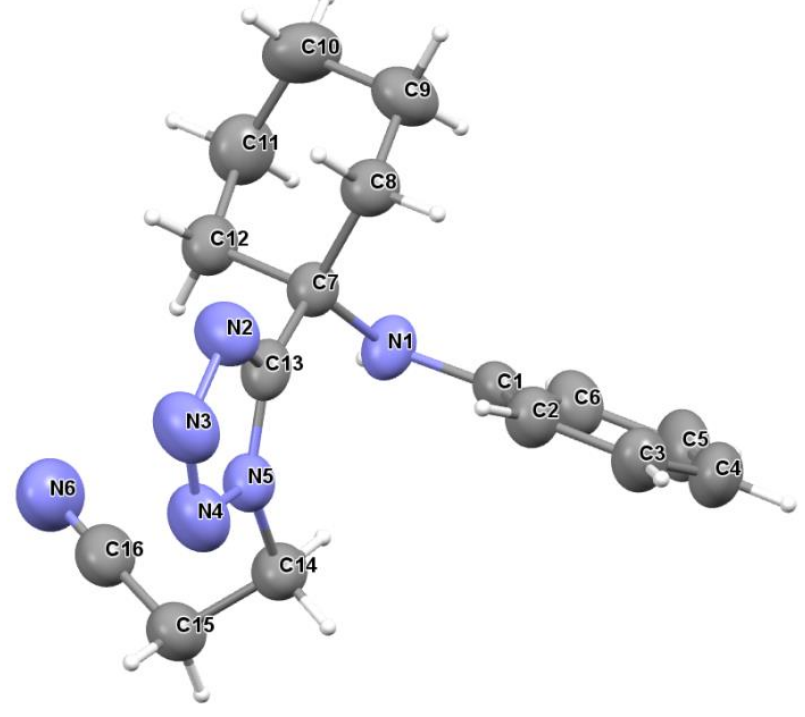

5c

Figure S1. Molecular geometry observed in the crystal structures of compounds 5c (asymmetric unit here), showing the atom labelling scheme. Displacement ellipsoids of non-hydrogen atoms are drawn at the $30 \%$ probability level. $\mathrm{H}$-atoms are presented as small spheres with an arbitrary radius. 


\section{References}

(1) Schrumpf, G.; Martin, S. J. Mol. Struct. 1983, 101, 57-67.

(2) CrysAlisPRO, Oxford Diffraction /Agilent Technologies UK Ltd, Yarnton, England, Version 1.171.36.20 (release 27-06-2012 CrysAlis171.NET)

(3) Burla, M.C.; Caliandro, R.; Camalli, M.; Carrozzini, B.; Cascarano, G.L.; De Caro, L.; Giacovazzo, C.; Polidori, G.; Spagna, R. J. Appl. Crystallogr. 2005, 38, 381-388.

(4) Sheldrick, G.M. Acta Crystallogr. 2008, A64, 112-122.

(5) Farrugia, L.J. J. Appl. Crystallogr. 1999, 32, 837-838.

(6) Macrae C.F.; Edgington P.R.; McCabe P.; Pidcock E.; Shields G.P.; Taylor R.; Towler M.; Van de Streek J. J. Appl. Crystallogr. 2006, 39, 453-457. 